\title{
Smoothness of solutions of a convolution equation of restricted type on the sphere
}

\author{
Diogo Oliveira e Silva ${ }^{1}$ and René Quilodrán ${ }^{2}$ \\ ${ }^{1}$ School of Mathematics, University of Birmingham, Edgbaston, B15 2TT,England; E-mail: d.oliveiraesilva@bham.ac.uk. \\ ${ }^{2}$ Santiago, Chile; E-mail: rquilodr@dim.uchile.cl.
}

Received: 3 June 2020; Revised: 15 December 2020; Accepted: 25 December 2020

2020 Mathematics Subject Classification: Primary - 49N60; Secondary - 35B38; 42B37

\section{Abstract}

Let $\mathbb{S}^{d-1}$ denote the unit sphere in Euclidean space $\mathbb{R}^{d}, d \geqslant 2$, equipped with surface measure $\sigma_{d-1}$. An instance of our main result concerns the regularity of solutions of the convolution equation

$$
\left.a \cdot\left(f \sigma_{d-1}\right)^{*(q-1)}\right|_{\mathbb{S} d-1}=f \text {, a.e. on } \mathbb{S}^{d-1},
$$

where $a \in C^{\infty}\left(\mathbb{S}^{d-1}\right), q \geqslant 2(d+1) /(d-1)$ is an integer, and the only a priori assumption is $f \in L^{2}\left(\mathbb{S}^{d-1}\right)$. We prove that any such solution belongs to the class $C^{\infty}\left(\mathbb{S}^{d-1}\right)$. In particular, we show that all critical points associated with the sharp form of the corresponding adjoint Fourier restriction inequality on $\mathbb{S}^{d-1}$ are $C^{\infty}$-smooth. This extends previous work of Christ and Shao [4] to arbitrary dimensions and general even exponents and plays a key role in the companion paper [24].

\section{Introduction}

Sharp Fourier restriction theory has attracted a great deal of interest recently. In the particular case of the unit sphere equipped with surface measure $\left(\mathbb{S}^{d-1}, \sigma_{d-1}\right)$, a natural starting point is that of the Tomas-Stein inequality,

$$
\left\|\widehat{f \sigma}_{d-1}\right\|_{L^{q}\left(\mathbb{R}^{d}\right)} \leqslant \mathbf{T}_{d, q}\|f\|_{L^{2}\left(\mathbb{S}^{d-1}\right)},
$$

which is known to hold [28, 29] with $\mathbf{T}_{d, q}<\infty$ provided that $d \geqslant 2$ and $q \geqslant q_{d}:=2 \frac{d+1}{d-1}$; see (1.3) for the precise definition of the Fourier extension operator. Here $\mathbf{T}_{d, q}$ denotes the optimal constant given by

$$
\mathbf{T}_{d, q}=\sup _{\mathbf{0} \neq f \in L^{2}} \frac{\left\|\widehat{f \sigma}_{d-1}\right\|_{L^{q}\left(\mathbb{R}^{d}\right)}}{\|f\|_{L^{2}\left(\mathbb{S}^{d-1}\right)}}
$$

By a maximiser of (1.1) we mean a nonzero, complex-valued function $f \in L^{2}\left(\mathbb{S}^{d-1}\right)$ for which $\left\|\widehat{f \sigma}_{d-1}\right\|_{L^{q}\left(\mathbb{R}^{d}\right)}=\mathbf{T}_{d, q}\|f\|_{L^{2}\left(\mathbb{S}^{d-1}\right)}$.

The existence of maximisers for the Tomas-Stein inequality (1.1) has been investigated in the works $[3,9,13,25]$, but the explicit form of the maximisers is only known in very few, special cases [1, 11]. Once maximisers are known to exist, it is natural to investigate their properties with methods from the calculus of variations. In the present article, we study the associated Euler-Lagrange equation and show

(C) The Author(s), 2021. Published by Cambridge University Press. This is an Open Access article, distributed under the terms of the Creative Commons Attribution licence (http://creativecommons.org/licenses/by/4.0/), which permits unrestricted re-use, distribution, and reproduction in any medium, provided the original work is properly cited. 
that the corresponding critical points are $C^{\infty}$-smooth whenever the exponent $q$ is an even integer. Our motivation is twofold. On the one hand, our main result is used in the companion paper [24] to establish that constant functions are the unique real-valued maximisers for a number of new sharp instances of inequality (1.1) and to fully characterise all complex-valued maximisers. On the other hand, we extend the main results of Christ and Shao [4] to arbitrary dimensions and general even exponents.

Let $d \geqslant 2$ and $q \geqslant q_{d}$ be given. Consider the Fourier extension operator $\mathcal{E}(f)=\widehat{f \sigma}_{d-1}$, acting on functions $f: \mathbb{S}^{d-1} \rightarrow \mathbb{C}$ via

$$
\widehat{f \sigma}_{d-1}(x)=\int_{\mathbb{S}^{d-1}} f(\omega) e^{-i x \cdot \omega} \mathrm{d} \sigma_{d-1}(\omega) .
$$

The operator $\mathcal{E}$ is bounded from $L^{2}\left(\mathbb{S}^{d-1}\right)$ to $L^{q}\left(\mathbb{R}^{d}\right)$ in light of (1.1). Its adjoint equals the restriction operator $\mathcal{E}^{*}(g)=\left.g^{\vee}\right|_{\mathbb{S} d-1}$ and is bounded from $L^{q^{\prime}}\left(\mathbb{R}^{d}\right)$ to $L^{2}\left(\mathbb{S}^{d-1}\right)$; here, $q^{\prime}=q /(q-1)$ denotes the conjugate Lebesgue exponent of $q$. Suppose that $f$ maximises the functional $\Phi_{d, q}$ associated to (1.1),

$$
\Phi_{d, q}(f)=\frac{\left\|\widehat{f \sigma}_{d-1}\right\|_{L^{q}\left(\mathbb{R}^{d}\right)}^{q}}{\|f\|_{L^{2}\left(\mathbb{S}^{d-1}\right)}^{q}}
$$

and further assume $f$ to be $L^{2}$-normalised, $\|f\|_{L^{2}\left(\mathbb{S}^{d-1}\right)}=1$. We can then estimate the operator norm of the extension operator as follows:

$$
\begin{aligned}
\|\mathcal{E}\|_{L^{2} \rightarrow L^{q}}^{q} & =\|\mathcal{E}(f)\|_{L^{q}\left(\mathbb{R}^{d}\right)}^{q}=\left\langle|\mathcal{E}(f)|^{q-2} \mathcal{E}(f), \mathcal{E}(f)\right\rangle=\left\langle\mathcal{E}^{*}\left(|\mathcal{E}(f)|^{q-2} \mathcal{E}(f)\right), f\right\rangle_{L^{2}\left(\mathbb{S}^{d-1}\right)} \\
& \leqslant\left\|\mathcal{E}^{*}\left(|\mathcal{E}(f)|^{q-2} \mathcal{E}(f)\right)\right\|_{L^{2}\left(\mathbb{S}^{d-1}\right)} \leqslant\left\|\mathcal{E}^{*}\right\|_{L^{q^{\prime} \rightarrow L^{2}}}\left\||\mathcal{E}(f)|^{q-2} \mathcal{E}(f)\right\|_{L^{q^{\prime}}\left(\mathbb{R}^{d}\right)} \\
& =\left\|\mathcal{E}^{*}\right\|_{L^{q^{\prime} \rightarrow L^{2}}}\|\mathcal{E}(f)\|_{L^{q}\left(\mathbb{R}^{d}\right)}^{q-1}=\|\mathcal{E}\|_{L^{2} \rightarrow L^{q}}^{q},
\end{aligned}
$$

where $\langle\cdot, \cdot\rangle$ denotes the $L^{q^{\prime}}-L^{q}$ pairing in $\mathbb{R}^{d}$, and $\langle\cdot, \cdot\rangle_{L^{2}\left(\mathbb{S}^{d-1}\right)}$ denotes the $L^{2}$ pairing in $\mathbb{S}^{d-1}$. In addition to easy algebraic manipulations, the first inequality in (1.5) amounts to an application of the Cauchy-Schwarz inequality, and the second inequality in (1.5) holds because the adjoint operator $\mathcal{E}^{*}$ is bounded from $L^{q^{\prime}}$ to $L^{2}$. In the last identity, we also used the fact that the operator norms of $\mathcal{E}, \mathcal{E}^{*}$ coincide, $\|\mathcal{E}\|_{L^{2} \rightarrow L^{q}}=\left\|\mathcal{E}^{*}\right\|_{L^{q^{\prime} \rightarrow L^{2}}}$. Because the first and last terms in the chain of inequalities (1.5) coincide, all inequalities are forced to be equalities. In particular, equality holds in the application of the Cauchy-Schwarz inequality, which in turn implies the existence of a constant $\mu$, for which

$$
\mathcal{E}^{*}\left(|\mathcal{E}(f)|^{q-2} \mathcal{E}(f)\right)=\mu f
$$

holds outside a set of zero $\sigma_{d-1}$-measure. Thus, we see that a maximiser of (1.1) necessarily satisfies

$$
\left.\left(\left|\widehat{f \sigma}_{d-1}\right|^{q-2} \widehat{f \sigma}_{d-1}\right)^{\vee}\right|_{\mathbb{S}^{d-1}}=\lambda\|f\|_{L^{2}\left(\mathbb{S}^{d-1}\right)}^{q-2} f, \quad \sigma_{d-1} \text {-a.e. on } \mathbb{S}^{d-1},
$$

for some $\lambda \in \mathbb{C}$. This is the Euler-Lagrange equation associated with the variational problem (1.2); see [2] for a more general statement. To determine the parameter $\lambda \in \mathbb{C}$, one simply multiplies both sides of (1.6) by $\bar{f}$ and integrates with respect to surface measure to check that $\lambda=\Phi_{d, q}(f)$. In particular, $f$ is a maximiser of inequality (1.1) if and only if (1.6) holds with $\lambda=\mathbf{T}_{d, q}^{q}$.

General nonzero solutions of the Euler-Lagrange equation (1.6) are called critical points of the functional $\Phi_{d, q}$. As noted in [2], it follows at once that constant functions satisfy (1.6) for some $\lambda>0$, simply because $\left|\widehat{\sigma}_{d-1}\right|^{q-2} \widehat{\sigma}_{d-1}$ is a radial function, the inverse Fourier transform of any radial function is radial and the restriction of any radial function on $\mathbb{R}^{d}$ to $\mathbb{S}^{d-1}$ is constant. 
If $q=2 n$ is an even integer, $n \in \mathbb{N}$, then the Tomas-Stein inequality (1.1) can be equivalently stated in convolution form via Plancherel's theorem as

$$
\left\|\left(f \sigma_{d-1}\right)^{* n}\right\|_{L^{2}\left(\mathbb{R}^{d}\right)}^{2} \leqslant(2 \pi)^{-d} \mathbf{T}_{d, 2 n}^{2 n}\|f\|_{L^{2}\left(\mathbb{S}^{d-1}\right)}^{2 n},
$$

where the $n$-fold convolution measure $\left(f \sigma_{d-1}\right)^{* n}$ is recursively defined for integral values of $n \geqslant 2$ via

$$
\left(f \sigma_{d-1}\right)^{* 2}=f \sigma_{d-1} * f \sigma_{d-1}, \text { and }\left(f \sigma_{d-1}\right)^{*(n+1)}=\left(f \sigma_{d-1}\right)^{* n} * f \sigma_{d-1} .
$$

The functional $\Phi_{d, 2 n}$ can then be rewritten as

$$
\Phi_{d, 2 n}(f)=(2 \pi)^{d} \frac{\left\|\left(f \sigma_{d-1}\right)^{* n}\right\|_{L^{2}\left(\mathbb{R}^{d}\right)}^{2}}{\|f\|_{L^{2}\left(\mathbb{S}^{d-1}\right)}^{2 n}},
$$

and the Euler-Lagrange equation (1.6) translates to

$$
\left.\left(\left(f \sigma_{d-1}\right)^{* n} *\left(f_{\star} \sigma_{d-1}\right)^{*(n-1)}\right)\right|_{\mathbb{S}_{d-1}}=(2 \pi)^{-d} \lambda\|f\|_{L^{2}\left(\mathbb{S}^{d-1}\right)}^{2 n-2} f, \quad \sigma_{d-1} \text {-a.e. on } \mathbb{S}^{d-1},
$$

where $f_{\star}$ denotes the conjugate reflection of $f$ around the origin, defined via

$$
f_{\star}(\omega)=\overline{f(-\omega)}, \quad \text { for all } \omega \in \mathbb{S}^{d-1} .
$$

A function $f: \mathbb{S}^{d-1} \rightarrow \mathbb{C}$ is said to be antipodally symmetric if $f=f_{\star}$, in which case basic properties of the Fourier transform imply that $\widehat{f \sigma}_{d-1}$ is real valued.

The convolution structure of equation (1.10) induces some extra regularity on its solutions, a phenomenon that turns out to hold in greater generality. To describe it precisely, consider the multilinear operator $\mathrm{M}: L^{2}\left(\mathbb{S}^{d-1}\right)^{m+1} \rightarrow L^{2}\left(\mathbb{S}^{d-1}\right)$,

$$
\mathrm{M}\left(f_{1}, \ldots, f_{m+1}\right)=\left.\left(f_{1} \sigma_{d-1} * \cdots * f_{m+1} \sigma_{d-1}\right)\right|_{\mathbb{S} d-1},
$$

which is well defined for integral values of $m \geqslant 4$ if $d=2$ and $m \geqslant 2$ if $d \geqslant 3$ in view of the chain of inequalities (1.5); see also [2, Prop. 2.4]. Further consider the conjugate reflection operator $R: L^{2}\left(\mathbb{S}^{d-1}\right) \rightarrow L^{2}\left(\mathbb{S}^{d-1}\right), R(f)=f_{\star}$. Given an integer $k \in \mathbb{N}_{0}$, the powers $R^{k}$ are defined in the usual way via composition, with the understanding that $R^{0}=\mathrm{Id}$. We are interested in solutions of the general equation

$$
a \cdot \mathrm{M}\left(R^{k_{1}}(f), \ldots, R^{k_{m+1}}(f)\right)=\lambda f, \quad \sigma_{d-1} \text {-a.e. on } \mathbb{S}^{d-1},
$$

where $\left(k_{1}, \ldots, k_{m+1}\right) \in\{0,1\}^{m+1}, a \in C^{\infty}\left(\mathbb{S}^{d-1}\right)$ and $\lambda \in \mathbb{C}$. The additional factor $a \in C^{\infty}\left(\mathbb{S}^{d-1}\right)$ brings no further complications to the analysis but can be used to address the smoothness of critical points for weighted measures on $\mathbb{S}^{d-1}$ and, by an additional scaling argument, on ellipsoids.

Our main result concerns regularity properties of generic solutions of equation (1.12).

Theorem 1.1. Let $d \geqslant 2$, and let $m$ be an integer satisfying $m \geqslant 4$ if $d=2$ and $m \geqslant 2$ if $d \geqslant 3$. Let $\left(k_{1}, \ldots, k_{m+1}\right) \in\{0,1\}^{m+1}, a \in C^{\infty}\left(\mathbb{S}^{d-1}\right)$ and $\lambda \in \mathbb{C} \backslash\{0\}$. If $f \in L^{2}\left(\mathbb{S}^{d-1}\right)$ is a complex-valued solution of equation (1.12), then $f \in C^{\infty}\left(\mathbb{S}^{d-1}\right)$.

The special case $(d, m)=(3,2)$ of Theorem 1.1 implies [4, Theorem 1.1]. Thus, Theorem 1.1 extends [4, Theorem 1.1] to arbitrary dimensions and general even exponents. Interestingly, our proof of Theorem 1.1 bypasses the Banach fixed point argument from [4] and, as such, could be considered more elementary and of independent value. Moreover, the case $(d, m)=(2,4)$ of Theorem 1.1 completes the proof of the main result in [26], where the following issue was detected: In [26, Proof of Prop. 3.6], the first (unnumbered) displayed equation on page 9 seems to be incorrect. We further believe that the argument 
in [26] cannot be repaired without studying the regularity of the fourfold convolution $\sigma_{1}^{* 4}$, such as a Hölder-type estimate of the kind established in Subsection 4.3. The following result is an immediate consequence of Theorem 1.1 and is used in a crucial manner in the companion paper [24].

Corollary 1.2. Let $d \geqslant 2$ and $q \geqslant 2 \frac{d+1}{d-1}$ be an even integer. If $f \in L^{2}\left(\mathbb{S}^{d-1}\right)$ is a critical point of the functional $\Phi_{d, q}$, then $f \in C^{\infty}\left(\mathbb{S}^{d-1}\right)$. In particular, maximisers of $\Phi_{d, q}$ are $C^{\infty}$-smooth.

\subsection{Outline}

In Section 2 we recall some useful facts about the special orthogonal group and define the appropriate smoothness spaces on $\mathbb{S}^{d-1}$ on which our estimates will be based. In Section 3 we collect some simple properties of the multilinear operator $\mathbf{M}$, defined in (1.11). A fundamental distinction arises, depending on whether or not the parameters $(d, m)$ from Theorem 1.1 lie on the 'boundary' of the set of admissible values. In the latter case, there is an automatic uniform gain in the initial regularity, which leads to a quick proof of the smoothing property of M in the 'non-boundary' case; see Lemma 3.4. This is not possible if $(d, m)$ lies on the boundary, because in that case the corresponding functional is essentially scale invariant. The analysis is then more delicate and relies on Hölder-type estimates for certain convolution operators, which are the subject of Section 4. In turn, these estimates are used in Section 5 to find a suitable replacement for Lemma 3.4 in the boundary case; see Lemma 5.2. The final section, Section 6, is devoted to the proof of Theorem 1.1. We proceed in two steps: firstly, we establish an initial 'kick' in the regularity of any solution of equation (1.12); secondly, we use a bootstrapping procedure to promote the initial gain in regularity to $C^{\infty}$-smoothness.

\subsection{Notation}

The set of natural numbers is $\mathbb{N}=\{1,2,3, \ldots\}$, and $\mathbb{N}_{0}=\mathbb{N} \cup\{0\}$. Given a set $E \subset \mathbb{R}^{d}$, its indicator function is denoted by $\mathbb{1}_{E}$, its Lebesgue measure by $|E|$ and its complement by $E^{\mathcal{C}}=\mathbb{R}^{d} \backslash E$. Given $r>0$, we let $B(x, r) \subset \mathbb{R}^{d}$ denote the closed ball of radius $r$ centered at $x \in \mathbb{R}^{d}$ and abbreviate $B_{r}=B(0, r)$. We will continue to denote by $\left(f \sigma_{d-1}\right)^{* k}$ the $k$-fold convolution measure, recursively defined in (1.8). We denote $\mathbf{1}: \mathbb{S}^{d-1} \rightarrow \mathbb{R}$ the function $\mathbf{1}(\omega) \equiv 1$ and the zero function by $\mathbf{0}: \mathbb{S}^{d-1} \rightarrow \mathbb{R}, \mathbf{0}(\omega) \equiv 0$. We use $X \lesssim Y, Y \gtrsim X$ or $X=O(Y)$ to denote the estimate $|X| \leqslant C Y$ for an absolute constant $C$ and $X \simeq Y$ to denote the estimates $X \lesssim Y \lesssim X$. We will often require the implied constant $C$ in the above notation to depend on additional parameters, which we will indicate by subscripts (unless explicitly omitted); thus, for instance, $X \lessgtr_{j} Y$ denotes an estimate of the form $|X| \leqslant C_{j} Y$ for some $C_{j}$ depending on $j$.

\section{Function spaces}

The special orthogonal group $\mathrm{SO}(d)$ consists of all $d \times d$ orthogonal matrices of unit determinant and acts transitively on the unit sphere $\mathbb{S}^{d-1}$ in the natural way. This action extends to actions on functions $f: \mathbb{S}^{d-1} \rightarrow \mathbb{C}$ by $\Theta f=f \circ \Theta$ for $\Theta \in \mathrm{SO}(d)$ and on finite Borel measures $\mu$ on $\mathbb{R}^{d}$ by $\Theta(\mu)(E)=\mu(\Theta(E))$ for $E \subseteq \mathbb{R}^{d}$. This extension interacts well with convolutions, in the sense that $\Theta(\mu * v)=\Theta(\mu) * \Theta(v)$. In particular, for any $\Theta \in \operatorname{SO}(d)$,

$$
\Theta\left(f_{1} \sigma_{d-1} * \cdots * f_{k} \sigma_{d-1}\right)=\left(\Theta f_{1}\right) \sigma_{d-1} * \cdots *\left(\Theta f_{k}\right) \sigma_{d-1} .
$$

For further information on the special orthogonal group, see [17] and references therein.

Given $\alpha \in(0,1)$, let $\Lambda_{\alpha}\left(\mathbb{R}^{d}\right)$ denote the space of Hölder continuous functions $f: \mathbb{R}^{d} \rightarrow \mathbb{C}$ of order $\alpha$, with norm

$$
\|f\|_{\Lambda_{\alpha}\left(\mathbb{R}^{d}\right)}=\|f\|_{C^{0}\left(\mathbb{R}^{d}\right)}+\sup _{x \neq x^{\prime}}\left|x-x^{\prime}\right|^{-\alpha}\left|f(x)-f\left(x^{\prime}\right)\right| .
$$

Given $1<\alpha \notin \mathbb{N}$, write $\alpha=k+\delta$, with $k \in \mathbb{N}$ and $\delta \in(0,1)$. We then say that $f \in \Lambda_{\alpha}\left(\mathbb{R}^{d}\right)$ if $f$ is $k$ times continuously differentiable, $f \in C^{k}\left(\mathbb{R}^{d}\right)$ and all of the $k$ th-order partial derivatives of $f$ belong 
to $\Lambda_{\delta}\left(\mathbb{R}^{d}\right)$. An equivalent definition of the space $\Lambda_{\alpha}\left(\mathbb{R}^{d}\right)$ via Littlewood-Paley projections is available, but we shall delay its precise formulation until the need arises in the proof of Proposition 3.1. Given $\alpha \in(0,1)$, the space of Hölder continuous functions $f: \mathbb{S}^{d-1} \rightarrow \mathbb{C}$ of order $\alpha$, denoted $\Lambda_{\alpha}\left(\mathbb{S}^{d-1}\right)$, is defined in a similar way to (2.2). We further consider the $\operatorname{space} \operatorname{Lip}\left(\mathbb{S}^{d-1}\right)$ of $\operatorname{Lipschitz}$ continuous functions $f: \mathbb{S}^{d-1} \rightarrow \mathbb{C}$, equipped with the norm

$$
\|f\|_{\operatorname{Lip}\left(\mathbb{S}^{d-1}\right)}=\|f\|_{C^{0}\left(\mathbb{S}^{d-1}\right)}+\sup _{\omega \neq \omega^{\prime}}\left|\omega-\omega^{\prime}\right|^{-1}\left|f(\omega)-f\left(\omega^{\prime}\right)\right| .
$$

By $H^{s}=H^{s}\left(\mathbb{S}^{d-1}\right)$ we mean the usual Sobolev space of functions having $s \geqslant 0$ derivatives in $L^{2}\left(\mathbb{S}^{d-1}\right)$, defined via spherical harmonic expansions - for example, as in [22, §1.7.3, Remark 7.6] - or by considering a smooth partition of unity and diffeomorphisms onto the unit ball in $\mathbb{R}^{d-1}$ together with the usual Sobolev norm on $\mathbb{R}^{d-1}$; we set $H^{0}=L^{2}\left(\mathbb{S}^{d-1}\right)$. If $s$ is an integer, then the following norm is equivalent to any other norm for $H^{s}$ :

$$
\|f\|_{H^{s}}=\|f\|_{L^{2}(\mathbb{S} d-1)}+\sum_{1 \leqslant i<j \leqslant d}\left\|X_{i, j}^{s} f\right\|_{L^{2}(\mathbb{S} d-1)}
$$

where the derivatives are given by

$$
X_{i, j}=x_{i} \partial_{j}-x_{j} \partial_{i}=\frac{\partial}{\partial \theta_{i, j}}, X_{i, j}^{s}=\frac{\partial^{s}}{\partial \theta_{i, j}^{s}},
$$

and $\theta_{i, j}$ denotes the angle in polar coordinates of the $\left(x_{i}, x_{j}\right)$-plane; see, for instance, [6, $\left.\S 4.5\right]$, and [8, Prop. 3.3].

We find it convenient to work with the function spaces $\mathcal{H}^{s}=\mathcal{H}^{s}\left(\mathbb{S}^{d-1}\right)$, which for $d=3$ were introduced in [4]. To extend the definition to general dimensions $d \geqslant 2$, recall (2.4), where we introduced the derivatives $X_{i, j}=\partial / \partial \theta_{i, j}$. We can equivalently view $X_{i, j}$ as the $C^{\infty}$-vector field on $\mathbb{S}^{d-1}$ that generates rotations about the $\left(x_{i}, x_{j}\right)$-plane for each $1 \leqslant i<j \leqslant d$. In this way, for each $v=$ $\left(v_{1}, \ldots, v_{d}\right) \in \mathbb{S}^{d-1}, \exp \left(t X_{i, j}\right)(v)$ is obtained by rotating the vector $\left(v_{i}, v_{j}\right)$ by $t$ radians. We note that $\left\{X_{i, j}: 1 \leqslant i<j \leqslant d\right\}$ forms a basis for $\mathfrak{s} \mathfrak{v}(d)$, the Lie algebra of $S O(d)$.

Observe that the following quantity defines an equivalent norm on the space $\Lambda_{\alpha}\left(\mathbb{S}^{d-1}\right)$, provided $\alpha \in(0,1)$ :

$$
\|f\|_{C^{0}\left(\mathbb{S}^{d-1}\right)}+\max _{1 \leqslant i<j \leqslant d} \sup _{\omega \in \mathbb{S}^{d-1}} \sup _{t \in \mathbb{R}}|t|^{-\alpha}\left|f\left(e^{t X_{i, j}}(\omega)\right)-f(\omega)\right|
$$

Given $s \in(0,1)$, the space $\mathcal{H}^{s}$ is defined as the set of all functions $f \in L^{2}\left(\mathbb{S}^{d-1}\right)$ for which the norm

$$
\|f\|_{\mathcal{H}^{s}}=\|f\|_{L^{2}\left(\mathbb{S}^{d-1}\right)}+\sum_{1 \leqslant i<j \leqslant d} \sup _{|t| \leqslant 1}|t|^{-s}\left\|f \circ e^{t X_{i, j}}-f\right\|_{L^{2}\left(\mathbb{S}^{d-1}\right)}
$$

is finite. We further set $\mathcal{H}^{0}=L^{2}\left(\mathbb{S}^{d-1}\right)$. Similar to the case of Euclidean space, the notion of weak differentiability of a function with respect to the vector field $X_{i, j}$ is made precise by the use of identity [24, Eq. (5.4)], which states that, for any complex-valued functions $f, g \in C^{1}\left(\mathbb{S}^{d-1}\right)$,

$$
\int_{\mathbb{S} d-1}\left(X_{i, j} f\right) \bar{g} \mathrm{~d} \sigma_{d-1}=-\int_{\mathbb{S} d-1} f \overline{\left(X_{i, j} g\right)} \mathrm{d} \sigma_{d-1} .
$$

In this way, we say that $f \in L^{2}\left(\mathbb{S}^{d-1}\right)$ is weakly differentiable with respect to the vector field $X_{i, j}$ if there exists a function, denoted $X_{i, j} f$, that belongs to $L^{1}\left(\mathbb{S}^{d-1}\right)$ and satisfies (2.6) for all $g \in C^{\infty}\left(\mathbb{S}^{d-1}\right)$. 
If $s=k+\alpha$, with $k \in \mathbb{N}$ and $\alpha \in(0,1)$, then the space $\mathcal{H}^{s}$ consists of all functions $f \in L^{2}\left(\mathbb{S}^{d-1}\right)$ for which the norm

$$
\|f\|_{\mathcal{H}^{s}}=\|f\|_{L^{2}\left(\mathbb{S}^{d-1}\right)}+\sum_{Y} \sum_{1 \leqslant i<j \leqslant d} \sup _{|t| \leqslant 1}|t|^{-\alpha}\left\|Y f \circ e^{t X_{i, j}}-Y f\right\|_{L^{2}\left(\mathbb{S}^{d-1}\right)}
$$

is finite, where $Y$ ranges over the finite set of all compositions $X_{i_{1}, j_{1}} \circ X_{i_{2}, j_{2}} \circ \cdots \circ X_{i_{\ell}, j_{\ell}}$ with $0 \leqslant \ell \leqslant k$ factors, and $f$ itself is viewed as $Y f$ where $Y$ has zero factors. We implicitly assume the function $f$ to be weakly differentiable with respect to the vector fields $\left\{X_{i, j}\right\}_{1 \leqslant i<j \leqslant d}$ as many times as required by the definition of the norm.

The next result explores the relationship between the function spaces $\mathcal{H}^{s}$ and the usual Sobolev spaces $H^{t}$.

Lemma 2.1. For every $0 \leqslant t<s, s \notin \mathbb{N}, \mathcal{H}^{s}$ is contained in the Sobolev space $H^{t}$, and

$$
\|f\|_{H^{t}} \leqslant C(s, t)\|f\|_{\mathcal{H}^{s}},
$$

for all $f \in \mathcal{H}^{s}$ and some constant $C(s, t)<\infty$.

Estimate (2.8) was noted in [4, Lemma 2.1] in the three-dimensional case $d=3$ when $s<1$. From Lemma 2.1 it follows at once that, given $s \in(1, \infty) \backslash \mathbb{N}, f \in \mathcal{H}^{s}$ and $X \in\left\{X_{i, j}: 1 \leqslant i<j \leqslant d\right\}$, then $\|X f\|_{L^{2}\left(\mathbb{S}^{d-1}\right)} \lesssim\|f\|_{\mathcal{H}^{s}}$ and therefore $X f \in \mathcal{H}^{s-1}$. This observation will be useful in the sequel.

As a preliminary step towards the proof of Lemma 2.1, we recall the Euclidean Sobolev spaces $H^{s}\left(\mathbb{R}^{d}\right)$ and define the spaces $\mathcal{H}^{s}\left(\mathbb{R}^{d}\right)$ in analogy to the spherical ones, $\mathcal{H}^{s}$. Given $f: \mathbb{R}^{d} \rightarrow \mathbb{R}$ and $s=k+\alpha$ with $k \in \mathbb{N}_{0}$ and $\alpha \in(0,1)$, we consider the norms

$$
\begin{gathered}
\|f\|_{H^{s}\left(\mathbb{R}^{d}\right)}^{2}:=\int_{\mathbb{R}^{d}}\left(1+|\xi|^{2}\right)^{s}|\widehat{f}(\xi)|^{2} \mathrm{~d} \xi, \\
\|f\|_{\mathcal{H}^{s}\left(\mathbb{R}^{d}\right)}:=\|f\|_{L^{2}\left(\mathbb{R}^{d}\right)}+\sum_{\ell} \sup _{|x| \leqslant 1}|x|^{-\alpha}\left\|D^{\ell} f \circ \tau_{x}-D^{\ell} f\right\|_{L^{2}\left(\mathbb{R}^{d}\right)},
\end{gathered}
$$

where the sum in (2.10) runs over all multi-indices $\ell=\left(\ell_{1}, \ldots, \ell_{d}\right) \in \mathbb{N}^{d}$ satisfying $0 \leqslant|\ell| \leqslant k$, $D^{\ell}:=\partial^{|\ell|} / \partial x_{d}^{\ell_{d}} \cdots \partial x_{1}^{\ell_{1}}$ denotes the partial derivative, $\tau_{x}: \mathbb{R}^{d} \rightarrow \mathbb{R}^{d}, y \mapsto x+y$ denotes translation by $x=\left(x_{1}, \ldots, x_{d}\right) \in \mathbb{R}^{d}$ and $f$ itself is viewed as $D^{0} f$. For $1 \leqslant i \leqslant d$, let $e_{i}$ denote the $i$ th canonical vector $e_{i}=(0, \ldots, 0,1,0, \ldots, 0)$, with the 1 in the $i$ th position. For every $s=k+\alpha, k \in \mathbb{N}_{0}, \alpha \in(0,1)$, the following is an equivalent norm for $\mathcal{H}^{s}\left(\mathbb{R}^{d}\right)$, perhaps more reminiscent to that for $\mathbb{S}^{d-1}$ in $(2.7)$ :

$$
\|f\|_{L^{2}\left(\mathbb{R}^{d}\right)}+\sum_{0 \leqslant|\ell| \leqslant k} \sum_{1 \leqslant i \leqslant d} \sup _{|t| \leqslant 1}|t|^{-\alpha}\left\|D^{\ell} f \circ \tau_{t e_{i}}-D^{\ell} f\right\|_{L^{2}\left(\mathbb{R}^{d}\right)} .
$$

It is also worth observing that, by the triangle inequality and the translation invariance of the Lebesgue measure in $\mathbb{R}^{d}$, an equivalent norm to that in (2.10) or (2.11) is obtained by replacing sup $|t| \leqslant 1$ by $\sup _{|t| \leqslant \varepsilon}$ for any $\varepsilon>0$. Likewise, by the triangle inequality and the $S O(d)$-invariance of the measure $\sigma_{d-1}$ in $\mathbb{S}^{d-1}$, an equivalent norm for $\mathcal{H}^{s}$ is obtained from (2.7) by replacing $\sup _{|t| \leqslant 1}$ by $\sup _{|t| \leqslant \varepsilon}$ for any $\varepsilon>0$.

Proof of Lemma 2.1. We discuss the analogous Euclidean statement for the case of the sphere then follows by working in local coordinates. In fact, as already mentioned, the $H^{t}$-norm on $\mathbb{S}^{d-1}$ can be defined by considering a smooth partition of unity and diffeomorphisms onto the unit ball in $\mathbb{R}^{d-1}$ together with the usual Sobolev norm on $\mathbb{R}^{d-1}$, as in (2.9). In order to handle the $\mathcal{H}^{s}$-norm on $\mathbb{S}^{d-1}$, we observe that it is likewise amenable to the use of local coordinates: Given a smooth partition of unity $\left\{\varphi_{i}\right\}_{1 \leqslant i \leqslant N}$ on $\mathbb{S}^{d-1}, f \in \mathcal{H}^{s}$ if and only if $\varphi_{i} f \in \mathcal{H}^{s}$ for every $1 \leqslant i \leqslant N$, and $\|f\|_{\mathcal{H}^{s}} \simeq \sum_{1 \leqslant i \leqslant N}\left\|\varphi_{i} f\right\|_{\mathcal{H}^{s}}$. Let $O_{i}$ denote the support of $\varphi_{i}$, which we may take to be connected and of small diameter if necessary, and let $\left\{\left(\Omega_{i}, \psi_{i}\right)\right\}_{1 \leqslant i \leqslant N}$ denote a system of local coordinates for $\mathbb{S}^{d-1}$ subordinate to $\left\{O_{i}\right\}_{1 \leqslant i \leqslant N}$; that 
is, $\Omega_{i}$ is open and connected, $\psi_{i}: \Omega_{i} \rightarrow \operatorname{int}\left(B_{1}\right)$ is a diffeomorphism onto the open unit ball in $\mathbb{R}^{d-1}$ and $O_{i} \Subset \Omega_{i}$ is compactly contained in $\Omega_{i}$. If $\varepsilon>0$ is small enough, it then follows that $\left\|\varphi_{i} f\right\|_{L^{2}\left(\mathbb{S}^{d-1}\right)}=\left\|\varphi_{i} f\right\|_{L^{2}\left(\Omega_{i}\right)}$ and, for every $|t| \leqslant \varepsilon$,

$$
\left\|Y\left(\varphi_{i} f\right) \circ e^{t X_{k, l}}-Y\left(\varphi_{i} f\right)\right\|_{L^{2}(\mathbb{S} d-1)}=\left\|Y\left(\varphi_{i} f\right) \circ e^{t X_{k, l}}-Y\left(\varphi_{i} f\right)\right\|_{L^{2}\left(\Omega_{i} \cap e^{\left.-t X_{k, l}\left(\Omega_{i}\right)\right)}\right.},
$$

for every $Y$ and $(k, l)$ as in (2.10). In this way, in order to show that $\left\|\varphi_{i} f\right\|_{\mathcal{H}^{s}} \simeq\left\|\left(\varphi_{i} f\right) \circ \psi_{i}^{-1}\right\|_{\mathcal{H}^{s}\left(\mathbb{R}^{d-1}\right)}$, one may appeal to the theory of differentiability along noncommuting vector fields, as developed in [20, $\S 4]$; see, in particular, Lemmas 4.1, 4.2 and Theorem 4.3 in [20].

In light of the previous paragraph, we can assume that in the Euclidean case the relevant supports are contained in the unit ball of $\mathbb{R}^{d}$. This will be useful later on in the argument. More precisely, the task is now to show that there exists $C(s, t)<\infty$ such that for every $f \in \mathcal{H}^{s}\left(\mathbb{R}^{d}\right)$ whose support is contained in the unit ball of $\mathbb{R}^{d}$, it holds that

$$
\|f\|_{H^{t}\left(\mathbb{R}^{d}\right)} \leqslant C(s, t)\|f\|_{\mathcal{H}^{s}\left(\mathbb{R}^{d}\right)},
$$

whenever $0 \leqslant t<s \notin \mathbb{N}$.

We start by considering the case $0<t<s<1$ (the case $t=0$ being trivial) and recalling the equivalent formulation of Sobolev spaces in terms of the Riesz potential. Fix $t \in(0,1)$, and let $f: \mathbb{R}^{d} \rightarrow \mathbb{R}$ be given. From Plancherel's theorem, we have that

$$
\begin{aligned}
\int_{\left(\mathbb{R}^{d}\right)^{2}} \frac{|f(x+y)-f(y)|^{2}}{|x|^{d+2 t}} \mathrm{~d} x \mathrm{~d} y & =\int_{\mathbb{R}^{d}} \int_{\mathbb{R}^{d}}\left|e^{i x \cdot \xi}-1\right|^{2}|\widehat{f}(\xi)|^{2} \mathrm{~d} \xi \frac{\mathrm{d} x}{|x|^{d+2 t}} \\
& =A_{t, d} \int_{\mathbb{R}^{d}}|\xi|^{2 t}|\widehat{f}(\xi)|^{2} \mathrm{~d} \xi
\end{aligned}
$$

where we used the fact that the integral

$$
I_{t, d}(\xi):=\int_{\mathbb{R}^{d}} \frac{\left|e^{i x \cdot \xi}-1\right|^{2}}{|x|^{d+2 t}} \mathrm{~d} x
$$

satisfies $I_{t, d}(\lambda \xi)=\lambda^{2 t} I_{t, d}(\xi)$ for every $\lambda>0$. The constant $A_{t, d}$ in (2.14) satisfies $A_{t, d}=I_{t, d}(\omega)$ for any $\omega \in \mathbb{S}^{d-1}$ and is finite as long as $t \in(0,1)$. In turn, because $t \in(0,1)$, we have that

$$
\left(1+|\xi|^{2}\right)^{t} \leqslant 1+|\xi|^{2 t} \leqslant 2\left(1+|\xi|^{2}\right)^{t}
$$

for every $\xi \in \mathbb{R}^{d}$. In particular, the following two-sided estimate holds:

$$
\|f\|_{H^{t}\left(\mathbb{R}^{d}\right)}^{2} \simeq_{t, d}\|f\|_{L^{2}\left(\mathbb{R}^{d}\right)}^{2}+\int_{\left(\mathbb{R}^{d}\right)^{2}} \frac{|f(x+y)-f(y)|^{2}}{|x|^{d+2 t}} \mathrm{~d} x \mathrm{~d} y .
$$

Given $s \in(t, 1)$, we use Hölder's inequality to estimate

$$
\begin{aligned}
\int_{\left(\mathbb{R}^{d}\right)^{2}} & \frac{|f(x+y)-f(y)|^{2}}{|x|^{d+2 t}} \mathrm{~d} x \mathrm{~d} y \\
& =\int_{|x| \leqslant 1} \int_{\mathbb{R}^{d}} \frac{|f(x+y)-f(y)|^{2}}{|x|^{d+2 t}} \mathrm{~d} y \mathrm{~d} x+\int_{|x|>1} \int_{\mathbb{R}^{d}} \frac{|f(x+y)-f(y)|^{2}}{|x|^{d+2 t}} \mathrm{~d} y \mathrm{~d} x \\
& \leqslant\left(\sup _{|x| \leqslant 1} \int_{\mathbb{R}^{d}} \frac{|f(x+y)-f(y)|^{2}}{|x|^{2 s}} \mathrm{~d} y\right) \int_{|x| \leqslant 1} \frac{\mathrm{d} x}{|x|^{d-2(s-t)}}
\end{aligned}
$$




$$
\begin{gathered}
+2 \int_{|x|>1} \int_{\mathbb{R}^{d}} \frac{|f(x+y)|^{2}+|f(y)|^{2}}{|x|^{d+2 t}} \mathrm{~d} y \mathrm{~d} x \\
\lesssim_{d}(s-t)^{-1}\|f\|_{\mathcal{H}^{s}\left(\mathbb{R}^{d}\right)}^{2}+t^{-1}\|f\|_{L^{2}\left(\mathbb{R}^{d}\right)}^{2} .
\end{gathered}
$$

In light of (2.15), this establishes (2.12) in the particular case when $0<t<s<1$.

We now consider the case when $s=k+\alpha$, with $k \in \mathbb{N}$ and $\alpha \in(0,1)$. No generality is lost in assuming that $t \in(k, s)$ and specialising to the case $k=1$, so that the desired conclusion would follow from the estimate $\left\|D^{\ell} f\right\|_{H^{t-1}\left(\mathbb{R}^{d}\right)} \lesssim\|f\|_{\mathcal{H}^{s}\left(\mathbb{R}^{d}\right)}$ for every $|\ell| \leqslant 1$. In this case, the previous argument applies to $D^{\ell} f$ provided that $D^{\ell} f \in L^{2}\left(\mathbb{R}^{d}\right)$ for any $|\ell|=1$, with appropriately bounded $L^{2}\left(\mathbb{R}^{d}\right)$-norm, which we now verify in the special case when the support of $f$ is contained in the unit ball of $\mathbb{R}^{d}$. As discussed above, this will suffice for our application to $\mathbb{S}^{d-1}$.

Fix a multi-index $\ell \in \mathbb{N}^{d},|\ell|=1$, and write $D:=D^{\ell}$. Let $g \in C_{0}^{\infty}\left(\mathbb{R}^{d}\right)$ be such that $\operatorname{supp}(g) \subset B_{1}$. Then $(D g)(y-t \ell)=-\frac{\mathrm{d}}{\mathrm{d} t}(g(y-t \ell))=D(g(\cdot-t \ell))(y)$. By Fubini's theorem and the definition of weak derivative of $f$, it follows that

$$
0=\int_{-2}^{2} \int_{\mathbb{R}^{d}} \overline{(D g)}(y-t \ell) f(y) \mathrm{d} y \mathrm{~d} t=-\int_{-2}^{2} \int_{\mathbb{R}^{d}} \bar{g}(y)(D f)(y+t \ell) \mathrm{d} y \mathrm{~d} t .
$$

As a consequence,

$$
\int_{\mathbb{R}^{d}} D f(y) \bar{g}(y) \mathrm{d} y=\frac{1}{4} \int_{-2}^{2} \int_{\mathbb{R}^{d}}(D f(y)-D f(y+t \ell)) \bar{g}(y) \mathrm{d} y \mathrm{~d} t .
$$

By adding and subtracting appropriate terms, the triangle and Cauchy-Schwarz inequalities and the invariance of the Lebesgue measure in $\mathbb{R}^{d}$ with respect to translations together imply

$$
\begin{aligned}
\left|\int_{\mathbb{R}^{d}} D f(y) \bar{g}(y) \mathrm{d} y\right| \leqslant & \frac{1}{4} \int_{-2}^{2}\left\|D f \circ \tau_{t \ell}-D f \circ \tau_{\frac{t}{2} \ell}\right\|_{L^{2}\left(\mathbb{R}^{d}\right)}\|g\|_{L^{2}\left(\mathbb{R}^{d}\right)} \mathrm{d} t \\
& +\frac{1}{4} \int_{-2}^{2}\left\|D f \circ \tau_{\frac{t}{2} \ell}-D f\right\|_{L^{2}\left(\mathbb{R}^{d}\right)}\|g\|_{L^{2}\left(\mathbb{R}^{d}\right)} \mathrm{d} t \\
& \lesssim \int_{0}^{1}\left\|D f \circ \tau_{t \ell}-D f\right\|_{L^{2}\left(\mathbb{R}^{d}\right)} \mathrm{d} t\|g\|_{L^{2}\left(\mathbb{R}^{d}\right)} \\
& \leqslant\left(\int_{0}^{1} t^{\alpha} \mathrm{d} t\right)\|f\|_{\mathcal{H}^{1+\alpha}\left(\mathbb{R}^{d}\right)}\|g\|_{L^{2}\left(\mathbb{R}^{d}\right)} .
\end{aligned}
$$

Consequently, $D f \in L^{2}\left(\mathbb{R}^{d}\right)$ and $\|D f\|_{L^{2}\left(\mathbb{R}^{d}\right)} \lesssim\|f\|_{\mathcal{H}^{s}\left(\mathbb{R}^{d}\right)}$. This concludes the proof of the lemma.

Remark 2.2. For our purposes later on, it will suffice to invoke the following simpler consequence of Lemma 2.1: For any $0<s \notin \mathbb{N}$, there is a continuous embedding $\mathcal{H}^{s} \subseteq H^{\lfloor s\rfloor}$. We now provide a short proof of this fact that is intrinsic to the sphere. The case $s \in(0,1)$ is clear because then $H^{\lfloor s\rfloor}=L^{2}\left(\mathbb{S}^{d-1}\right)$. For $s>1, s \notin \mathbb{N}$ and $f \in \mathcal{H}^{s}$ it suffices to show that $\|X f\|_{L^{2}\left(\mathbb{S}^{d-1}\right)} \lesssim\|f\|_{\mathcal{H}^{s}}$, where $X$ ranges over the finite set of all compositions $X_{i_{1}, j_{1}} \circ X_{i_{2}, j_{2}} \circ \cdots \circ X_{i_{\ell}, j_{\ell}}$ with $1 \leqslant \ell \leqslant\lfloor s\rfloor$ factors. ${ }^{1}$ As noted in the course of the proof of Lemma 2.1, we may specialise to the case $\ell=1$ because the general case follows in the same way. We then simply note that, for any $g \in C^{\infty}\left(\mathbb{S}^{d-1}\right), X \in\left\{X_{i, j}: 1 \leqslant i<j \leqslant d\right\}$ and $\omega \in \mathbb{S}^{d-1}$, it holds that $(X g)\left(e^{t X} \omega\right)=\left(X\left(g \circ e^{t X}\right)\right)(\omega)=\frac{d}{d t}\left(g\left(e^{t X} \omega\right)\right)$, so that $\int_{0}^{2 \pi}(X g)\left(e^{t X} \omega\right) \mathrm{d} t=0$, and the desired estimate,

$$
\left|\int_{\mathbb{S}^{d-1}} X f(\omega) \bar{g}(\omega) \mathrm{d} \sigma_{d-1}(\omega)\right| \lesssim\|f\|_{\mathcal{H}^{s}}\|g\|_{L^{2}\left(\mathbb{S}^{d-1}\right)},
$$

${ }^{1}$ As stated in (2.3) and explained in the references thereafter, it suffices to consider the case $\ell=\lfloor s\rfloor$. 
follows in the same way as (2.16). See also [5, Cor. 7] for a discussion of this embedding using an equivalent definition ${ }^{2}$ of $\mathcal{H}^{s}$.

\section{Preliminary inequalities}

We establish some linear and multilinear inequalities that will be used to analyse the solutions of equation (1.12). Our first result translates into a modest amount of control over the regularity of convolution measures in a number of situations of interest.

Proposition 3.1. Given integers $d, m \geqslant 2$, set $\alpha=\frac{1}{2}(d-1)(m-2)-1$. Let $\left\{f_{j}\right\}_{j=1}^{m} \subset C^{\infty}\left(\mathbb{S}^{d-1}\right)$. If $\alpha>0$, then $f_{1} \sigma_{d-1} * \cdots * f_{m} \sigma_{d-1} \in \Lambda_{\alpha}\left(\mathbb{R}^{d}\right)$.

The proof of Proposition 3.1 is based on the classical Littlewood-Paley characterisation of the Hölder spaces $\Lambda_{\alpha}\left(\mathbb{R}^{d}\right)$; see $[14, \S 6.3]$ and [28, Ch. VI, §5].

Proof of Proposition 3.1. Consider a smooth partition of unity in $\mathbb{R}^{d}$. More precisely, fix $\eta \geqslant 0$, a nonnegative, decreasing and radial $C^{\infty}$-function of compact support, defined on $\mathbb{R}^{d}$, with the properties that $\eta(x)=1$ for $|x| \leqslant 1$ and $\eta(x)=0$ for $|x| \geqslant 2$. Together with $\eta$, define another function $\delta$ by $\delta(x):=\eta(x)-\eta(2 x) \geqslant 0$. For each integer $j \geqslant 1$, consider the function $\varphi_{j}:=\delta\left(2^{-j}.\right)$, which is supported on the spherical shell $\left\{x \in \mathbb{R}^{d}: 2^{j-1} \leqslant|x| \leqslant 2^{j+1}\right\}$, and let $\varphi_{0}=\eta$, so that

$$
\sum_{j=0}^{\infty} \varphi_{j}(x)=1, \quad \text { for every } x \in \mathbb{R}^{d} .
$$

For $\alpha>0$, a function $G: \mathbb{R}^{d} \rightarrow \mathbb{C}$ belongs to $\Lambda_{\alpha}\left(\mathbb{R}^{d}\right)$ if and only if

$$
\sup _{j \in \mathbb{N}_{0}} 2^{j \alpha}\left\|\left(\widehat{G} \varphi_{j}\right)^{\vee}\right\|_{L^{\infty}\left(\mathbb{R}^{d}\right)}<\infty .
$$

Moreover, the expression on the left-hand side of (3.1) produces a norm that is equivalent to any other norm for $\Lambda_{\alpha}\left(\mathbb{R}^{d}\right)$; see [14, Theorem 6.3.7]. The Hausdorff-Young inequality implies that estimate (3.1) is fulfilled if

$$
\int_{\mathbb{R}^{d}}\left|\widehat{G}(x) \varphi_{j}(x)\right| \mathrm{d} x \lesssim 2^{-j \alpha}, \quad j=0,1,2, \ldots,
$$

for some implicit constant that does not depend on $j$. Now, the Fourier transform of $F:=f_{1} \sigma_{d-1} * \cdots *$ $f_{m} \sigma_{d-1}$ is given by $\widehat{F}=\prod_{k=1}^{m} \widehat{f}_{k} \sigma_{d-1}$, which leads to the analysis of the integrals

$$
\int_{B_{2}} \prod_{k=1}^{m}\left|{\widehat{f_{k} \sigma}}_{d-1}(x)\right| \mathrm{d} x, \quad \int_{B_{2^{j+1}} \backslash B_{2^{j-1}}} \prod_{k=1}^{m}\left|{\widehat{f_{k} \sigma-1}}_{d}(x)\right| \mathrm{d} x, \quad j=1,2, \ldots
$$

A well-known stationary phase argument applied to each $f_{k} \in C^{\infty}\left(\mathbb{S}^{d-1}\right)$ yields the following decay estimate:

$$
\left|\widehat{f}_{k} \sigma_{d-1}(x)\right| \lesssim(1+|x|)^{-\frac{d-1}{2}}, \text { for every } x \in \mathbb{R}^{d},
$$

where the implicit constant depends only on the dimension $d$ and the function $f_{k}$; see [28, Chapter VIII, $\S 3.1]$. Using polar coordinates, it is then direct to check that

$$
\int_{B_{2^{j+1}} \backslash B_{2^{j-1}}} \prod_{k=1}^{m}\left|\widehat{f}_{k} \sigma_{d-1}(x)\right| \mathrm{d} x \lesssim 2^{j d} 2^{-\frac{j m(d-1)}{2}}=2^{-j\left((d-1)\left(\frac{m}{2}-1\right)-1\right)},
$$

${ }^{2}$ We comment on various equivalent definitions of the space $\mathcal{H}^{s}$ in Subsection 6.2. 
for every $j \in \mathbb{N}$. The desired conclusion follows from this and from the observation that $\widehat{F}$ defines a continuous function on $\mathbb{R}^{d}$ and is thus bounded on the ball $B_{2} \subset \mathbb{R}^{d}$.

Remark 3.2. We find it convenient to consider the 'universe' of admissible parameters

$$
\mathfrak{U}=\left\{(d, m) \in \mathbb{N}^{2}: d=2 \text { and } m \geqslant 4 \text {, or } d \geqslant 3 \text { and } m \geqslant 2\right\},
$$

together with its 'boundary'

$$
\partial \mathfrak{U}=\{(2,4),(3,3)\} \cup\{(d, 2): d \geqslant 3\} .
$$

Note that the set $\mathfrak{U}$ encapsulates the hypotheses on $d, m$ imposed by Theorem 1.1. On the other hand, with the exception of $(d, m)=(3,3)$, the set $\partial \mathfrak{U}$ contains precisely those values $(d, m)$ for which $m$ is the smallest even integer such that $\mathbf{T}_{d, m+2}<\infty$, and therefore the corresponding inequality (1.1) holds. As the upcoming sections will reveal, the analysis simplifies considerably if $(d, m) \in \mathfrak{U} \backslash \partial \mathfrak{U}$, which is the reason for treating the boundary set $\partial \mathfrak{U}$ separately. As a first instance of this phenomenon, note that, given $(d, m) \in \mathfrak{U}$, we have that $(d, m) \notin \partial \mathfrak{U}$ if and only if $\frac{1}{2}(d-1)(m-2)-1>0$. These are precisely the cases covered by Proposition 3.1. See also the comments following Lemma 3.4 and Remark 6.4.

Recall the operator $\mathrm{M}: L^{2}\left(\mathbb{S}^{d-1}\right)^{m+1} \rightarrow L^{2}\left(\mathbb{S}^{d-1}\right)$, which was defined in (1.11) as

$$
\mathbf{M}\left(f_{1}, \ldots, f_{m+1}\right)=\left.\left(f_{1} \sigma_{d-1} * \cdots * f_{m+1} \sigma_{d-1}\right)\right|_{\mathbb{S} d-1}
$$

Lemma 3.3. The operator $\mathrm{M}$ defined in (1.11) satisfies the following properties:

(i) $\mathrm{M}$ is an $(m+1)$-linear operator.

(ii) $\mathrm{M}$ is symmetric in the sense that, given any permutation $\tau$ of $\{1,2, \ldots, m+1\}$,

$$
\mathbf{M}\left(f_{1}, \ldots, f_{m+1}\right)=\mathbf{M}\left(f_{\tau(1)}, \ldots, f_{\tau(m+1)}\right) .
$$

(iii) For any $\Theta \in S O(d)$, the following identities hold:

$$
\begin{aligned}
\mathrm{M}\left(f_{1}, \ldots, f_{m+1}\right) \circ \Theta= & \mathrm{M}\left(f_{1} \circ \Theta, \ldots, f_{m+1} \circ \Theta\right) \\
(\Theta-I) \mathrm{M}\left(f_{1}, \ldots, f_{m+1}\right)= & \sum_{j=1}^{m+1} \mathrm{M}\left(f_{1}, \ldots, f_{j-1},(\Theta-I) f_{j}, \Theta f_{j+1}, \ldots, \Theta f_{m+1}\right) \\
= & \mathrm{M}\left((\Theta-I) f_{1}, \Theta f_{2}, \ldots, \Theta f_{m+1}\right) \\
& +\mathrm{M}\left(f_{1},(\Theta-I) f_{2}, \ldots, \Theta f_{m+1}\right) \\
& \vdots \\
& +\mathrm{M}\left(f_{1}, f_{2}, \ldots,(\Theta-I) f_{m+1}\right) .
\end{aligned}
$$

(iv) For any $s \geqslant 0$, there exists $A_{s}<\infty$ such that if $\left\{f_{j}\right\}_{j=1}^{m+1} \subset H^{s}$, then

$$
\left\|\mathrm{M}\left(f_{1}, \ldots, f_{m+1}\right)\right\|_{H^{s}} \leqslant A_{s} \prod_{j=1}^{m+1}\left\|f_{j}\right\|_{H^{s}} .
$$


(v) If $X=X_{i, j}^{3}$ for some $1 \leqslant i<j \leqslant d$ and $\left\{f_{k}\right\}_{k=1}^{m+1} \subset H^{1}$, then

$$
X \mathrm{M}\left(f_{1}, \ldots, f_{m+1}\right)=\sum_{k=1}^{m+1} \mathrm{M}\left(f_{1}, \ldots, f_{k-1}, X f_{k}, f_{k+1}, \ldots, f_{m+1}\right) \text {. }
$$

(vi) For any $0<s \notin \mathbb{Z}$, there exists $C_{s}<\infty$ such that, if $\left\{f_{j}\right\}_{j=1}^{m+1} \subset \mathcal{H}^{s}$, then

$$
\left\|\mathbf{M}\left(f_{1}, \ldots, f_{m+1}\right)\right\|_{\mathcal{H}^{s}} \leqslant C_{s} \prod_{j=1}^{m+1}\left\|f_{j}\right\|_{\mathcal{H}^{s}}
$$

We record the basic $L^{2}$-estimate, which coincides with the case $s=0$ of (3.6):

$$
\left\|\mathbf{M}\left(f_{1}, \ldots, f_{m+1}\right)\right\|_{L^{2}(\mathbb{S} d-1)} \lesssim \prod_{j=1}^{m+1}\left\|f_{j}\right\|_{L^{2}(\mathbb{S} d-1)} .
$$

Proof of Lemma 3.3. We prove estimate (3.8) only, the rest being direct from the definitions or simple to verify; in particular, the proof of (iv) is analogous to that of [4, Lemma 2.2]. Let us first assume that $s \in(0,1)$. Given $\left\{f_{k}\right\}_{k=1}^{m+1} \subset \mathcal{H}^{s}$, set $g:=\mathrm{M}\left(f_{1}, \ldots f_{m+1}\right)$. Let $\Theta=e^{t X} \in \mathrm{SO}(d)$, where $X=X_{i, j}$ for some $1 \leqslant i<j \leqslant d$. In light of (3.5), we then have that

$$
\Theta g-g=\sum_{k=1}^{m+1} \mathrm{M}\left(f_{1}, \ldots, f_{k-1},(\Theta-I) f_{k}, \Theta f_{k+1}, \ldots, \Theta f_{m+1}\right) .
$$

By (3.9), the first summand on the right-hand side of (3.10) satisfies

$$
\left\|\mathrm{M}\left((\Theta-I) f_{1}, f_{2}, \ldots, f_{m+1}\right)\right\|_{L^{2}\left(\mathbb{S}^{d-1}\right)} \lesssim\left\|\Theta f_{1}-f_{1}\right\|_{L^{2}\left(\mathbb{S}^{d-1}\right)} \prod_{\ell=2}^{m+1}\left\|f_{\ell}\right\|_{L^{2}\left(\mathbb{S}^{d-1}\right)},
$$

and similarly for the other $m$ summands. It follows that

$$
\begin{aligned}
\sup _{|t| \leqslant 1}|t|^{-s}\left\|g \circ e^{t X}-g\right\|_{L^{2}(\mathbb{S} d-1)} & \lesssim \sum_{k=1}^{m+1} \sup _{|t| \leqslant 1}|t|^{-s}\left\|e^{t X} f_{k}-f_{k}\right\|_{L^{2}(\mathbb{S} d-1)} \prod_{\ell: \ell \neq k}\left\|f_{\ell}\right\|_{L^{2}\left(\mathbb{S}^{d-1}\right)} \\
& \leqslant \sum_{k=1}^{m+1}\left\|f_{k}\right\|_{\mathcal{H}^{s}} \prod_{\ell: \ell \neq k}\left\|f_{\ell}\right\|_{L^{2}\left(\mathbb{S}^{d-1}\right)} \leqslant \prod_{k=1}^{m+1}\left\|f_{k}\right\|_{\mathcal{H}^{s}} .
\end{aligned}
$$

Because this holds whenever $X$ is any of the vector fields $\left\{X_{i, j}\right\}_{1 \leqslant i<j \leqslant d}$, estimate (3.8) as follows, settling (vi) in the special case when $s \in(0,1)$. Now suppose that $s=k+\alpha$, with $k \in \mathbb{N}$ and $\alpha \in(0,1)$. Let $1 \leqslant \ell \leqslant k$ and consider a composition $Y$ with $\ell$ factors as in (2.7). Remark 2.2 and estimate (3.6) imply that $g \in H^{k}$. In light of (3.7), we then see that $Y g$ can be written as a sum of terms of the form $\mathrm{M}\left(Y_{1} f_{1}, \ldots, Y_{m+1} f_{m+1}\right)$, where $Y_{1}, \ldots, Y_{m+1}$ are compositions of $i_{1}, \ldots, i_{m+1}$ vector fields $X_{i, j}$, and $\sum_{j=1}^{m+1} i_{j}=\ell$. Note that $Y_{j} f_{j} \in \mathcal{H}^{\alpha}$ for all such vector fields, and $\left\|Y_{j} f_{j}\right\|_{\mathcal{H}^{\alpha}} \leqslant\left\|f_{j}\right\|_{\mathcal{H}^{s}}$. Expanding $(\Theta-I) Y g$ as in (3.10), we find in the same way as before that

$$
\sup _{|t| \leqslant 1}|t|^{-\alpha}\left\|Y g \circ e^{t X}-Y g\right\|_{L^{2}\left(\mathbb{S}^{d-1}\right)} \lesssim \prod_{j=1}^{m+1}\left\|f_{j}\right\|_{\mathcal{H}^{s}}
$$

This implies the desired $\mathcal{H}^{s}$-bound for the function $g$ and concludes the proof of the lemma.

${ }^{3}$ Recall the definition (2.4) of $X_{i, j}=\frac{\partial}{\partial \theta_{i, j}}$. 
The following result details a sense in which $\mathrm{M}$ can be viewed as a smoothing operator but requires $(d, m) \notin \partial \mathfrak{U}$.

Lemma 3.4. Given $(d, m) \in \mathfrak{U} \backslash \partial \mathfrak{U}$, set $\alpha_{d, m}=\frac{1}{2}(d-1)(m-2)-1$. If $\alpha \in(0,1)$ is such that $\alpha \leqslant \alpha_{d, m},\left\{\varphi_{j}\right\}_{j=1}^{m} \subset C^{\infty}\left(\mathbb{S}^{d-1}\right)$ and $g \in L^{2}\left(\mathbb{S}^{d-1}\right)$, then $\mathrm{M}\left(\varphi_{1}, \ldots, \varphi_{m}, g\right) \in \mathcal{H}^{\alpha}$. Moreover, the following estimate holds:

$$
\begin{aligned}
\left\|\mathrm{M}\left(\varphi_{1}, \ldots, \varphi_{m}, g\right)\right\|_{\mathcal{H}^{\alpha}} & \\
& \lesssim\left(\prod_{j=1}^{m}\left\|\varphi_{j}\right\|_{L^{2}\left(\mathbb{S}^{d-1}\right)}+\left\|\varphi_{1} \sigma_{d-1} * \cdots * \varphi_{m} \sigma_{d-1}\right\|_{\Lambda_{\alpha_{d, m}}\left(\mathbb{R}^{d}\right)}\right)\|g\|_{L^{2}(\mathbb{S} d-1)}
\end{aligned}
$$

It is natural to wonder whether a similar gain in regularity holds in the case when $(d, m) \in \partial \mathfrak{U}$. The (affirmative) answer is more subtle, and we postpone the discussion until Section 5; see Lemma 5.2.

Proof of Lemma 3.4. Recall that $\alpha_{d, m}>0$ because $(d, m) \in \mathfrak{U} \backslash \partial \mathfrak{U}$. It then follows from Proposition 3.1 that $\varphi_{1} \sigma_{d-1} * \cdots * \varphi_{m} \sigma_{d-1} \in \Lambda_{\alpha_{d, m}}\left(\mathbb{R}^{d}\right)$. For notational convenience, we shall only consider the special case when $\varphi_{j}=\varphi$, for all $j$. Given $\Theta \in \operatorname{SO}(d)$ and $\omega \in \mathbb{S}^{d-1}$, estimate

$$
\begin{aligned}
&|\mathrm{M}(\varphi, \ldots, \varphi, g) \circ \Theta(\omega)-\mathrm{M}(\varphi, \ldots, \varphi, g)(\omega)| \\
& \leqslant \int_{\mathbb{S}^{d-1}}\left|\left(\varphi \sigma_{d-1}\right)^{* m}(\Theta \omega-\eta)-\left(\varphi \sigma_{d-1}\right)^{* m}(\omega-\eta)\right||g(\eta)| \mathrm{d} \sigma_{d-1}(\eta) .
\end{aligned}
$$

If $\alpha \in(0,1)$ is such that $\alpha \leqslant \alpha_{d, m}$, then $\left(\varphi \sigma_{d-1}\right)^{* m} \in \Lambda_{\alpha}\left(\mathbb{R}^{d}\right)$ and, consequently,

$$
\begin{aligned}
\mid \mathrm{M}(\varphi, \ldots, \varphi, g) \circ \Theta(\omega)-\mathrm{M}(\varphi, \ldots, & , g)(\omega) \mid \\
& \left.\leqslant|(\Theta-I) \omega|^{\alpha}\left\|\left(\varphi \sigma_{d-1}\right)^{* m}\right\|_{\Lambda_{\alpha}\left(\mathbb{R}^{d}\right)}\|g\|_{L^{1}(\mathbb{S} d-1}\right) \\
& \lesssim|\Theta-I|^{\alpha}\left\|\left(\varphi \sigma_{d-1}\right)^{* m}\right\|_{\Lambda_{\alpha}\left(\mathbb{R}^{d}\right)}\|g\|_{L^{2}\left(\mathbb{S}^{d-1}\right)} .
\end{aligned}
$$

Letting $\Theta=e^{t X}$ for some $X \in\left\{X_{i, j}\right\}_{1 \leqslant i<j \leqslant d}$ and integrating the square of both sides of the latter estimate, we obtain

$$
\begin{aligned}
\sup _{|t| \leqslant 1}|t|^{-\alpha}\|\mathrm{M}(\varphi, \ldots, \varphi, g) \circ \Theta-\mathrm{M}(\varphi, \ldots, \varphi, g)\|_{L^{2}\left(\mathbb{S}^{d-1}\right)} & \\
& \lesssim \sup _{|t| \leqslant 1}|t|^{-\alpha}\left|e^{t X}-I\right|^{\alpha}\left\|\left(\varphi \sigma_{d-1}\right)^{* m}\right\|_{\Lambda_{\alpha}\left(\mathbb{R}^{d}\right)}\|g\|_{L^{2}\left(\mathbb{S}^{d-1}\right)}
\end{aligned}
$$

In turn, this and the basic $L^{2}$-estimate (3.9) together imply

$$
\|\mathrm{M}(\varphi, \ldots, \varphi, g)\|_{\mathcal{H}^{\alpha}} \lesssim\left(\|\varphi\|_{L^{2}\left(\mathbb{S}^{d-1}\right)}^{m}+\left\|\left(\varphi \sigma_{d-1}\right)^{* m}\right\|_{\Lambda_{\alpha}\left(\mathbb{R}^{d}\right)}\right)\|g\|_{L^{2}\left(\mathbb{S}^{d-1}\right)} .
$$

To obtain (3.11), simply rerun the argument with the $\varphi_{j}$ s in place of $\varphi$. This completes the proof of the lemma.

\section{Hölder regularity}

In this section, we prove Hölder-type estimates for certain convolution measures, which will pave the way towards finding a suitable replacement for Lemma 3.4 in the case when $(d, m) \in \partial \mathfrak{H}$. 


\subsection{Twofold convolutions}

The purpose of this subsection is to generalise [4, Lemma 2.3] to arbitrary dimensions $d \geqslant 2$. Though for the most part the analysis follows similar lines to those of [4], we include it for the sake of completeness. Start by recalling that the twofold convolution $\sigma_{d-1} * \sigma_{d-1}$ defines a measure supported on the ball $B_{2} \subset \mathbb{R}^{d}$, which is absolutely continuous with respect to the Lebesgue measure on $B_{2}$, and whose Radon-Nikodym derivative equals

$$
\left(\sigma_{d-1} * \sigma_{d-1}\right)(x)=\frac{\omega_{d-2}}{2^{d-3}} \frac{1}{|x|}\left(4-|x|^{2}\right)_{+}^{\frac{d-3}{2}} .
$$

Here, $\omega_{d-2}:=\sigma_{d-2}\left(\mathbb{S}^{d-2}\right)=2 \pi^{\frac{d-1}{2}} \Gamma\left(\frac{d-1}{2}\right)^{-1}$ denotes the surface area of $\mathbb{S}^{d-2}, y_{+}:=\max \{0, y\}$ for $y \in \mathbb{R}$, and

$$
\left(4-|x|^{2}\right)_{+}^{\frac{d-3}{2}}:=\left(\left(4-|x|^{2}\right)_{+}\right)^{\frac{d-3}{2}}
$$

see, for instance, [1, Lemma 5].

Let $h_{1}, h_{2} \in \operatorname{Lip}\left(\mathbb{S}^{d-1}\right)$. From [12, Appendix A.2], we know that the function $u_{12}$ defined by the relation $\left(h_{1} \sigma_{d-1} * h_{2} \sigma_{d-1}\right)(x)=u_{12}(x)\left(\sigma_{d-1} * \sigma_{d-1}\right)(x)$ for $0<|x| \leqslant 2$ and $u_{12}(x)=0$ for $|x|>2$ can be expressed as

$$
u_{12}(x)=f_{\Gamma_{x}} h_{1}(v) h_{2}(x-v) \mathrm{d} \sigma_{x}(v),
$$

where $\Gamma_{x}=\mathbb{S}^{d-1} \cap\left(x+\mathbb{S}^{d-1}\right)$, and $f$ denotes the averaged integral on the $(d-2)$-dimensional sphere $\Gamma_{x}$; see also [3] for a careful discussion of the case $d=3$.

The case $d=2$ merits some further remarks. In this case, if $0<|x|<2$, then $\Gamma_{x}$ consists of two points, which we identify with $\mathbb{S}^{0}$. Let $x^{\perp}$ be the $90^{\circ}$-counterclockwise rotation of $x$, so that $x^{\perp} \cdot x=0$ and $\left|x^{\perp}\right|=|x|$. Given $x \in B_{2} \backslash\{0\} \subset \mathbb{R}^{2}$, there exist unique-up-to-permutation $x_{1}, x_{2} \in \mathbb{S}^{1}$, such that $x=x_{1}+x_{2}$. The vectors $x_{1}, x_{2}$ are explicitly given by

$$
x_{1}=\frac{x}{2}+\left(1-\frac{|x|^{2}}{4}\right)^{\frac{1}{2}} \frac{x^{\perp}}{|x|}, \quad x_{2}=\frac{x}{2}-\left(1-\frac{|x|^{2}}{4}\right)^{\frac{1}{2}} \frac{x^{\perp}}{|x|} .
$$

Given $h_{1}, h_{2} \in \operatorname{Lip}\left(\mathbb{S}^{1}\right)$, the convolution $h_{1} \sigma_{1} * h_{2} \sigma_{1}$ can be written in the following way: If $0<|x| \leqslant 2$, then

$$
\left(h_{1} \sigma_{1} * h_{2} \sigma_{1}\right)(x)=2 \frac{h_{1}\left(x_{1}\right) h_{2}\left(x_{2}\right)+h_{1}\left(x_{2}\right) h_{2}\left(x_{1}\right)}{|x| \sqrt{4-|x|^{2}}},
$$

and for $|x|>2$ one obviously has that $\left(h_{1} \sigma_{1} * h_{2} \sigma_{1}\right)(x)=0$. In this case, identity (4.2) is then seen to reduce to

$$
u_{12}(x)=\frac{1}{2}\left(h_{1}\left(x_{1}\right) h_{2}\left(x_{2}\right)+h_{1}\left(x_{2}\right) h_{2}\left(x_{1}\right)\right), \text { if } 0<|x| \leqslant 2 .
$$

Lemma 4.1. Let $d \geqslant 2$ and $x, x^{\prime} \in B_{2} \backslash\{0\} \subset \mathbb{R}^{d}$. Then

$$
\left|u_{12}(x)-u_{12}\left(x^{\prime}\right)\right| \leqslant C\left\|h_{1}\right\|_{\operatorname{Lip}\left(\mathbb{S}^{d-1}\right)}\left\|h_{2}\right\|_{\operatorname{Lip}\left(\mathbb{S}^{d-1}\right)}\left(\left|x-x^{\prime}\right|^{1 / 2}+\left|\frac{x}{|x|}-\frac{x^{\prime}}{\left|x^{\prime}\right|}\right|\right),
$$

for some universal constant $C<\infty$.

Proof. The integral (4.2) defining $u_{12}$ can be equivalently written as

$$
u_{12}(x)=\omega_{d-2}^{-1} \int_{\mathbb{S}_{x}^{d-2}} h_{1}\left(\frac{x}{2}+\rho(x) \omega\right) h_{2}\left(\frac{x}{2}-\rho(x) \omega\right) \mathrm{d} \sigma_{d-2}(\omega),
$$


where the function $\rho \geqslant 0$ satisfies $\rho(x)^{2}+(|x| / 2)^{2}=1$, and the unit sphere $\mathbb{S}_{x}^{d-2}$ is contained in the $(d-1)$-dimensional subspace of $\mathbb{R}^{d}$ orthogonal to $x$ and is therefore parallel to the hyperplane containing $\Gamma_{x}$. It is elementary to check that $\left|\rho(x)-\rho\left(x^{\prime}\right)\right| \leqslant|| x|-| x^{\prime} \|^{1 / 2}$ for every $x, x^{\prime} \in B_{2} \backslash\{0\}$.

Let us start by considering the case $x^{\prime}=\lambda x$ for some $\lambda>0$. We then have that $\mathbb{S}_{x}^{d-1}=\mathbb{S}_{x^{\prime}}^{d-1}$, and so

$$
\left|\left(\frac{x}{2}+\rho(x) \omega\right)-\left(\frac{x^{\prime}}{2}+\rho\left(x^{\prime}\right) \omega\right)\right| \leqslant \frac{1}{2}\left|x-x^{\prime}\right|+\left|\rho(x)-\rho\left(x^{\prime}\right)\right| \lesssim\left|x-x^{\prime}\right|^{1 / 2} .
$$

In a similar way,

$$
\left|\left(\frac{x}{2}-\rho(x) \omega\right)-\left(\frac{x^{\prime}}{2}-\rho\left(x^{\prime}\right) \omega\right)\right| \lesssim\left|x-x^{\prime}\right|^{1 / 2}
$$

Denote $x_{1}=\frac{x}{2}+\rho(x) \omega, x_{2}=\frac{x}{2}-\rho(x) \omega$ and $\tilde{u}(x)=h_{1}\left(x_{1}\right) h_{2}\left(x_{2}\right)$. Because $h_{1}, h_{2}$ are Lipschitz functions, we have that

$$
\begin{aligned}
\left|\tilde{u}(x)-\tilde{u}\left(x^{\prime}\right)\right| & =\left|h_{1}\left(x_{1}\right) h_{2}\left(x_{2}\right)-h_{1}\left(x_{1}^{\prime}\right) h_{2}\left(x_{2}^{\prime}\right)\right| \\
& \leqslant\left|h_{2}\left(x_{2}\right) \| h_{1}\left(x_{1}\right)-h_{1}\left(x_{1}^{\prime}\right)\right|+\left|h_{1}\left(x_{1}^{\prime}\right)\right|\left|h_{2}\left(x_{2}\right)-h_{2}\left(x_{2}^{\prime}\right)\right| \\
& \leqslant\left\|h_{2}\right\|_{L^{\infty}}\left\|h_{1}\right\|_{\text {Lip }}\left|x_{1}-x_{1}^{\prime}\right|+\left\|h_{1}\right\|_{L^{\infty}}\left\|h_{2}\right\|_{\text {Lip }}\left|x_{2}-x_{2}^{\prime}\right| \\
& \lesssim\left\|h_{1}\right\|_{\text {Lip }}\left\|h_{2}\right\|_{\text {Lip }}\left|x-x^{\prime}\right|^{1 / 2} .
\end{aligned}
$$

It then follows by integration over $\mathbb{S}_{x}^{d-2}$ that

$$
\left|u_{12}(x)-u_{12}\left(x^{\prime}\right)\right| \lesssim\left\|h_{1}\right\|_{\text {Lip }}\left\|h_{2}\right\|_{\text {Lip }}\left|x-x^{\prime}\right|^{1 / 2} .
$$

We now consider the case $|x|=\left|x^{\prime}\right| \in(0,2]$. We then have that $\rho(x)=\rho\left(x^{\prime}\right)$. Let $\Theta \in \operatorname{SO}(d)$ denote a rotation that fixes the space $\left(\operatorname{span}\left\{x, x^{\prime}\right\}\right)^{\perp}$ and sends $x /|x|$ to $x^{\prime} /\left|x^{\prime}\right|$. It is not difficult to see that $|\Theta-I| \leqslant|x /| x\left|-x^{\prime} /\right| x^{\prime}||$. We can then write

$$
u_{12}\left(x^{\prime}\right)=\omega_{d-2}^{-1} \int_{\mathbb{S}_{x}^{d-2}} h_{1}\left(\frac{x^{\prime}}{2}+\rho(x) \Theta \omega\right) h_{2}\left(\frac{x^{\prime}}{2}-\rho\left(x^{\prime}\right) \Theta \omega\right) \mathrm{d} \sigma_{d-2}(\omega),
$$

so that, for $\epsilon \in\{-1,1\}$,

$$
\begin{aligned}
\left|\left(\frac{x}{2}+\epsilon \rho(x) \omega\right)-\left(\frac{x^{\prime}}{2}+\epsilon \rho\left(x^{\prime}\right) \Theta \omega\right)\right| & \leqslant \frac{1}{2}\left|x-x^{\prime}\right|+\rho(x)|(\Theta-I) \omega| \\
& \leqslant \frac{1}{2}\left|x-x^{\prime}\right|+\rho(x)\left|\frac{x}{|x|}-\frac{x^{\prime}}{\left|x^{\prime}\right|}\right| \\
& =\left(\rho(x)+\frac{|x|}{2}\right)\left|\frac{x}{|x|}-\frac{x^{\prime}}{\left|x^{\prime}\right|}\right| \lesssim\left|\frac{x}{|x|}-\frac{x^{\prime}}{\left|x^{\prime}\right|}\right| .
\end{aligned}
$$

Reasoning as before, we conclude that

$$
\left|u_{12}(x)-u_{12}\left(x^{\prime}\right)\right| \lesssim\left\|h_{1}\right\|_{\text {Lip }}\left\|h_{2}\right\|_{\text {Lip }}\left|\frac{x}{|x|}-\frac{x^{\prime}}{\left|x^{\prime}\right|}\right| .
$$

For general $x, x^{\prime} \in B_{2} \backslash\{0\}$ we proceed as follows. Let $y=|x| x^{\prime} /\left|x^{\prime}\right|$, so that $|y|=|x|$ and $x^{\prime}=\lambda y$ for $\lambda=\left|x^{\prime}\right| /|x|>0$. Then

$$
\begin{aligned}
\left|u_{12}(x)-u_{12}\left(x^{\prime}\right)\right| & \leqslant\left|u_{12}(x)-u_{12}(y)\right|+\left|u_{12}(y)-u_{12}\left(x^{\prime}\right)\right| \\
& \lesssim\left\|h_{1}\right\|_{\text {Lip }}\left\|h_{2}\right\|_{\text {Lip }}\left(\left|x^{\prime}-y\right|^{1 / 2}+\left|\frac{y}{|y|}-\frac{x}{|x|}\right|\right)
\end{aligned}
$$




$$
\begin{aligned}
& =\left\|h_{1}\right\|_{\text {Lip }}\left\|h_{2}\right\|_{\text {Lip }}\left(\left\|x|-| x^{\prime}\right\|^{1 / 2}+\left|\frac{x^{\prime}}{\left|x^{\prime}\right|}-\frac{x}{|x|}\right|\right) \\
& \leqslant\left\|h_{1}\right\|_{\text {Lip }}\left\|h_{2}\right\|_{\text {Lip }}\left(\left|x-x^{\prime}\right|^{1 / 2}+\left|\frac{x}{|x|}-\frac{x^{\prime}}{\left|x^{\prime}\right|}\right|\right) .
\end{aligned}
$$

This completes the proof of the lemma.

The following consequence of Lemma 4.1 will be useful in the forthcoming analysis.

Corollary 4.2. Let $d \geqslant 3$ and $x, x^{\prime} \in B_{2} \backslash\{0\} \subset \mathbb{R}^{d}$. Then

$$
\begin{aligned}
|| x\left|\left(h_{1} \sigma_{d-1} * h_{2} \sigma_{d-1}\right)(x)-\right| x^{\prime}\left|\left(h_{1} \sigma_{d-1} * h_{2} \sigma_{d-1}\right)\left(x^{\prime}\right)\right| & \\
& \leqslant C\left\|h_{1}\right\|_{\operatorname{Lip}\left(\mathbb{S}^{d-1}\right)}\left\|h_{2}\right\|_{\operatorname{Lip}\left(\mathbb{S}^{d-1}\right)}\left(\left|x-x^{\prime}\right|^{1 / 2}+\left|\frac{x}{|x|}-\frac{x^{\prime}}{\left|x^{\prime}\right|}\right|\right),
\end{aligned}
$$

for some universal constant $C<\infty$.

Proof. From (4.1) and (4.2), for $|x| \leqslant 2$ we have that

$$
|x|\left(h_{1} \sigma_{d-1} * h_{2} \sigma_{d-1}\right)(x)=2^{-d+3} \omega_{d-2}\left(4-|x|^{2}\right)^{\frac{d-3}{2}} u_{12}(x) .
$$

The function $\left(4-|x|^{2}\right)^{\frac{d-3}{2}} \mathbb{1}_{B_{2}}(x)$ belongs to $\Lambda_{1 / 2}\left(\mathbb{R}^{d}\right)$ if $d \geqslant 4$ and to $\Lambda_{1 / 2}\left(B_{2}\right)$ if $d=3$. The desired conclusion follows easily from this and Lemma 4.1.

\subsection{The case $(d, n)=(3,3)$}

In the course of this subsection only, we shall simplify the notation by writing $\mathrm{d} \sigma=\mathrm{d} \sigma_{2}$. Our goal is to establish a Hölder estimate for the threefold convolution $h_{1} \sigma * h_{2} \sigma * h_{3} \sigma$, where $\left\{h_{j}\right\}_{j=1}^{3}$ are Lipschitz functions on the unit sphere $\mathbb{S}^{2}$.

Proposition 4.3. Given $h_{1}, h_{2}, h_{3} \in \operatorname{Lip}\left(\mathbb{S}^{2}\right)$, let $H=h_{1} \sigma * h_{2} \sigma * h_{3} \sigma$. Then there exists a universal constant $C<\infty$ such that, for every $x, x^{\prime} \in \mathbb{R}^{3}$,

$$
\left|H(x)-H\left(x^{\prime}\right)\right| \leqslant C \prod_{j=1}^{3}\left\|h_{j}\right\|_{\operatorname{Lip}\left(\mathbb{S}^{2}\right)}\left|x-x^{\prime}\right|^{1 / 3} .
$$

Proof. By homogeneity, we may assume $\left\|h_{j}\right\|_{\text {Lip }}=1,1 \leqslant j \leqslant 3$. Because the function $H$ is compactly supported, it is enough to consider $x, x^{\prime} \in \mathbb{R}^{3}$ for which ${ }^{4}\left|x-x^{\prime}\right| \ll 1$. From (4.1) and (4.2), the function $u_{12}(x):=(2 \pi)^{-1}|x|\left(h_{1} \sigma * h_{2} \sigma\right)(x)$ is given by

$$
u_{12}(x)=f_{\Gamma_{x}} h_{1}(v) h_{2}(x-v) \mathrm{d} \sigma_{x}(v)
$$

where $\Gamma_{x}=\mathbb{S}^{2} \cap\left(x+\mathbb{S}^{2}\right)$. We further have that

$$
H(x)=\int_{\mathbb{S}^{2}}\left(h_{1} \sigma * h_{2} \sigma\right)(x-\omega) h_{3}(\omega) \mathrm{d} \sigma(\omega)=2 \pi \int_{\mathbb{S}^{2}} \frac{\mathbb{1}_{|x-\omega|<2}(\omega)}{|x-\omega|} u_{12}(x-\omega) h_{3}(\omega) \mathrm{d} \sigma(\omega),
$$

\footnotetext{
${ }^{4}$ We will write $\left|x-x^{\prime}\right| \ll 1$ to mean that the quantity $\left|x-x^{\prime}\right|$ is sufficiently small for the purposes of the corresponding proof. For instance, in the course of the proof of Proposition 4.3, we can and will assume that $\left|x-x^{\prime}\right| \leqslant 100^{-1}$.
} 
and so

$$
\begin{aligned}
(2 \pi)^{-1}\left(H(x)-H\left(x^{\prime}\right)\right)= & \int_{\mathbb{S}^{2}} \frac{\mathbb{1}_{\left|x^{\prime}-\omega\right|<2}(\omega)}{\left|x^{\prime}-\omega\right|}\left(u_{12}(x-\omega)-u_{12}\left(x^{\prime}-\omega\right)\right) h_{3}(\omega) \mathrm{d} \sigma(\omega) \\
& \quad+\int_{\mathbb{S}^{2}}\left(\frac{\mathbb{1}_{|x-\omega|<2}(\omega)}{|x-\omega|}-\frac{\mathbb{1}_{\left|x^{\prime}-\omega\right|<2}(\omega)}{\left|x^{\prime}-\omega\right|}\right) u_{12}(x-\omega) h_{3}(\omega) \mathrm{d} \sigma(\omega) .
\end{aligned}
$$

We denote the integrals on the right-hand side of the latter identity by $I$ and $I I$, respectively. We start by estimating the first integral.

Estimating $I$. The first step is to restrict the domain of integration to the region where $x-\omega, x^{\prime}-\omega \in$ $B_{2}$, plus a remainder, which is $O\left(\left|x-x^{\prime}\right|\right)$. With this purpose in mind, decompose $\mathbb{S}^{2}=U \cup U^{\prime} \cup V \cup W$, where

$$
\begin{gathered}
U:=\left\{\omega \in \mathbb{S}^{2}:\left|x^{\prime}-\omega\right|<2 \leqslant|x-\omega|\right\}, U^{\prime}:=\left\{\omega \in \mathbb{S}^{2}:|x-\omega|<2 \leqslant\left|x^{\prime}-\omega\right|\right\} \\
V:=\left\{\omega \in \mathbb{S}^{2}:|x-\omega|,\left|x^{\prime}-\omega\right|<2\right\}, W:=\left\{\omega \in \mathbb{S}^{2}: 2 \leqslant\left|x^{\prime}-\omega\right|,|x-\omega|\right\} .
\end{gathered}
$$

The integrand of $I$ vanishes on the region $U^{\prime} \cup W$, and so we are left to analyse the integrals over $U$ and $V$. We claim that $\sigma(U)=O\left(\left|x-x^{\prime}\right|\right)$. Indeed, if $\omega \in U$, then $\left|x^{\prime}-\omega\right|<2 \leqslant|x-\omega|$, so that as $\left|x^{\prime}-\omega\right| \geqslant|x-\omega|-\left|x-x^{\prime}\right| \geqslant 2-\left|x-x^{\prime}\right|$ we obtain

$$
U \subseteq\left\{\omega \in \mathbb{S}^{2}: 2-\left|x-x^{\prime}\right| \leqslant\left|x^{\prime}-\omega\right| \leqslant 2\right\}
$$

This shows that the region $U$ is contained in the intersection of $\mathbb{S}^{2}$ with a spherical shell of thickness $\left|x-x^{\prime}\right|$ centred at $x^{\prime}$. The claim follows. The contribution of $U$ to the integral $I$ can then be bounded in the following way:

$$
\int_{U} \frac{\mathbb{1}_{\left|x^{\prime}-\omega\right|<2}(\omega)}{\left|x^{\prime}-\omega\right|}\left|u_{12}\left(x^{\prime}-\omega\right) h_{3}(\omega)\right| \mathrm{d} \sigma(\omega) \leqslant \int_{U} \frac{\mathbb{1}_{\left|x^{\prime}-\omega\right|<2}(\omega)}{\left|x^{\prime}-\omega\right|} \mathrm{d} \sigma(\omega) .
$$

If $\omega \in U$, then $\left|x^{\prime}-\omega\right| \geqslant 2-\left|x-x^{\prime}\right|>1$ because $\left|x-x^{\prime}\right| \ll 1$. As a consequence, the latter integral can be crudely bounded as follows:

$$
\int_{U} \frac{\mathrm{d} \sigma(\omega)}{\left|x^{\prime}-\omega\right|} \leqslant \sigma(U) \lesssim\left|x-x^{\prime}\right|
$$

To handle the contribution of the region $V$, note that Lemma 4.1 implies the pointwise estimate

$$
\left|u_{12}(x-\omega)-u_{12}\left(x^{\prime}-\omega\right)\right| \lesssim\left|x-x^{\prime}\right|^{1 / 2}+\left|\frac{x-\omega}{|x-\omega|}-\frac{x^{\prime}-\omega}{\left|x^{\prime}-\omega\right|}\right| .
$$

The contribution of the region

$$
R:=\left\{\omega \in V:\left|\frac{x-\omega}{|x-\omega|}-\frac{x^{\prime}-\omega}{\left|x^{\prime}-\omega\right|}\right| \leqslant\left|x-x^{\prime}\right|^{1 / 2}\right\}
$$

to the integral $I$ is easy to estimate. In view of (4.7) and (4.8),

$$
\begin{aligned}
\mid \int_{R} \frac{\mathbb{1}_{\left|x^{\prime}-\omega\right|<2}(\omega)}{\left|x^{\prime}-\omega\right|} & \left(u_{12}(x-\omega)-u_{12}\left(x^{\prime}-\omega\right)\right) h_{3}(\omega) \mathrm{d} \sigma(\omega) \mid \\
& \lesssim\left(\int_{R} \frac{\mathbb{1}_{\left|x^{\prime}-\omega\right|<2}(\omega)}{\left|x^{\prime}-\omega\right|} \mathrm{d} \sigma(\omega)\right)\left|x-x^{\prime}\right|^{1 / 2} \lesssim\left|x-x^{\prime}\right|^{1 / 2}
\end{aligned}
$$


In the second estimate, we used the elementary fact that there exists a universal constant $C<\infty$ such that

$$
\int_{R} \frac{\mathbb{1}_{\left|x^{\prime}-\omega\right|<2}(\omega)}{\left|x^{\prime}-\omega\right|} \mathrm{d} \sigma(\omega) \leqslant \int_{\mathbb{S}^{2}} \frac{\mathrm{d} \sigma(\omega)}{\left|x^{\prime}-\omega\right|} \leqslant C<\infty,
$$

for all $x^{\prime} \in \mathbb{R}^{3}$. If $\omega \in V \backslash R$, then

$$
\left|x-x^{\prime}\right|^{1 / 2}<\left|\frac{x-\omega}{|x-\omega|}-\frac{x^{\prime}-\omega}{\left|x^{\prime}-\omega\right|}\right| \leqslant \frac{2|x-\omega|\left|x-x^{\prime}\right|}{|x-\omega|\left|x^{\prime}-\omega\right|},
$$

from which we obtain $\left|x^{\prime}-\omega\right| \leqslant 2\left|x-x^{\prime}\right|^{1 / 2}$. The contribution of this region can then be estimated as follows:

$$
\begin{aligned}
\mid \int_{V \backslash R} \frac{\mathbb{1}_{\left|x^{\prime}-\omega\right|<2}(\omega)}{\left|x^{\prime}-\omega\right|} & \left(u_{12}(x-\omega)-u_{12}\left(x^{\prime}-\omega\right)\right) h_{3}(\omega) \mathrm{d} \sigma(\omega) \mid \\
& \lesssim \int_{V \backslash R} \frac{\mathbb{1}_{\left|x^{\prime}-\omega\right|<2}(\omega)}{\left|x^{\prime}-\omega\right|}\left|\frac{x-\omega}{|x-\omega|}-\frac{x^{\prime}-\omega}{\left|x^{\prime}-\omega\right|}\right| h_{3}(\omega) \mathrm{d} \sigma(\omega) \\
& \lesssim\left(\int_{\mathbb{S}^{2} \cap B\left(x^{\prime}, 2\left|x-x^{\prime}\right|^{1 / 2}\right)} \frac{\mathbb{1}_{\left|x^{\prime}-\omega\right|<2}(\omega)}{\left|x^{\prime}-\omega\right|} \mathrm{d} \sigma(\omega)\right)\left\|h_{3}\right\|_{L^{\infty}} \\
& \lesssim\left|x-x^{\prime}\right|^{1 / 2} .
\end{aligned}
$$

From the third line to the fourth line, we used the fact that

$$
\phi\left(x^{\prime}\right):=\int_{\mathbb{S}^{2} \cap B\left(x^{\prime}, \varepsilon\right)} \frac{\mathrm{d} \sigma(\omega)}{\left|x^{\prime}-\omega\right|}
$$

defines a radial function of $x^{\prime}$ that satisfies

$$
\phi\left(x^{\prime}\right) \lesssim \sigma\left(\mathbb{S}^{2} \cap B\left(x^{\prime}, \varepsilon\right)\right)^{1 / 2} \lesssim \varepsilon .
$$

This concludes the verification of the bound $|I| \lesssim\left|x-x^{\prime}\right|^{1 / 2}$.

Estimating $I I$. The integral $I I$ is bounded by

$$
\int_{\mathbb{S}^{2}}\left|\frac{\mathbb{1}_{|x-\omega|<2}(\omega)}{|x-\omega|}-\frac{\mathbb{1}_{\left|x^{\prime}-\omega\right|<2}(\omega)}{\left|x^{\prime}-\omega\right|}\right| \mathrm{d} \sigma(\omega) .
$$

By symmetry, it is enough to consider

$$
\int_{T}\left(\frac{\mathbb{1}_{|x-\omega|<2}(\omega)}{|x-\omega|}-\frac{\mathbb{1}_{\left|x^{\prime}-\omega\right|<2}(\omega)}{\left|x^{\prime}-\omega\right|}\right) \mathrm{d} \sigma(\omega),
$$

where the integral is taken over the region

$$
T:=\left\{\omega \in \mathbb{S}^{2}: \frac{\mathbb{1}_{|x-\omega|<2}(\omega)}{|x-\omega|}>\frac{\mathbb{1}_{\left|x^{\prime}-\omega\right|<2}(\omega)}{\left|x^{\prime}-\omega\right|}\right\} .
$$

Decompose $T=U^{\prime \prime} \cup V^{\prime \prime}$, where

$$
\begin{aligned}
& U^{\prime \prime}:=\left\{\omega \in T:|x-\omega|<2 \leqslant\left|x^{\prime}-\omega\right|\right\} \\
& V^{\prime \prime}:=\left\{\omega \in T:|x-\omega|<\left|x^{\prime}-\omega\right|<2\right\} .
\end{aligned}
$$


We have that $U^{\prime \prime}=U^{\prime} \cap T$, and therefore $\sigma\left(U^{\prime \prime}\right)=O\left(\left|x-x^{\prime}\right|\right)$. Moreover,

$$
\int_{U^{\prime \prime}}\left(\frac{\mathbb{1}_{|x-\omega|<2}(\omega)}{|x-\omega|}-\frac{\mathbb{1}_{\left|x^{\prime}-\omega\right|<2}(\omega)}{\left|x^{\prime}-\omega\right|}\right) \mathrm{d} \sigma(\omega)=\int_{U^{\prime \prime}} \frac{\mathrm{d} \sigma(\omega)}{|x-\omega|} \lesssim\left|x-x^{\prime}\right|,
$$

where the last inequality follows as in (4.6). The contribution of the region $V^{\prime \prime}$ to the integral in (4.11) is slightly more delicate to estimate. We consider two cases as before. Outside the ball $\left|x^{\prime}-\omega\right| \geqslant\left|x-x^{\prime}\right|^{1 / 3}$, we use the estimate $|x-\omega| \geqslant\left|x^{\prime}-\omega\right|-\left|x-x^{\prime}\right| \gtrsim\left|x-x^{\prime}\right|^{1 / 3}$, which implies

$$
\begin{aligned}
\left|\frac{1}{|x-\omega|}-\frac{1}{\left|x^{\prime}-\omega\right|}\right| & =\left|\frac{\left|x^{\prime}-\omega\right|-|x-\omega|}{|x-\omega|\left|x^{\prime}-\omega\right|}\right| \leqslant \frac{\left|x^{\prime}-x\right|}{|x-\omega|\left|x^{\prime}-\omega\right|} \\
& \lesssim\left|x-x^{\prime}\right|^{-2 / 3}\left|x-x^{\prime}\right|=\left|x-x^{\prime}\right|^{1 / 3} .
\end{aligned}
$$

Inside the ball $\left|x^{\prime}-\omega\right| \leqslant\left|x-x^{\prime}\right|^{1 / 3}$, we also have $|x-\omega| \leqslant\left|x-x^{\prime}\right|^{1 / 3}$, as $\omega \in V^{\prime \prime}$. The contribution of this region to the integral in (4.11) is at most two times the integral

$$
\phi\left(x^{\prime}\right)=\int_{\mathbb{S}^{2} \cap B\left(x^{\prime}, \delta\right)} \frac{\mathrm{d} \sigma(\omega)}{\left|x^{\prime}-\omega\right|},
$$

where $\delta=\left|x-x^{\prime}\right|^{1 / 3}$. Proceeding as in (4.10), one is led to the bound $\phi\left(x^{\prime}\right) \lesssim \delta$, whence the term in question is $O\left(\left|x-x^{\prime}\right|^{1 / 3}\right)$. This establishes the bound $|I I| \lesssim\left|x-x^{\prime}\right|^{1 / 3}$. The proof of the proposition is now complete.

Remark 4.4. Proposition 4.3 implies that if $n \geqslant 4$, then $G_{n}:=h_{1} \sigma * \cdots * h_{n} \sigma \in \Lambda_{1 / 3}\left(\mathbb{R}^{3}\right)$ whenever $\left\{h_{j}\right\}_{j=1}^{3} \subset \operatorname{Lip}\left(\mathbb{S}^{2}\right)$ and $\left\{h_{j}\right\}_{j=4}^{n} \subset L^{1}\left(\mathbb{S}^{2}\right)$. This can be improved under the additional assumption $\left\{h_{j}\right\}_{j=1}^{n} \subset \operatorname{Lip}\left(\mathbb{S}^{2}\right)$, in which case we have, for instance, that $G_{6} \in \Lambda_{2 / 3}\left(\mathbb{R}^{3}\right)$. In dimensions $d \geqslant 4$, a similar argument to that in the proof of Proposition 4.3 shows that, if $\left\{h_{j}\right\}_{j=1}^{3} \subset \operatorname{Lip}\left(\mathbb{S}^{d-1}\right)$, then $h_{1} \sigma_{d-1} * h_{2} \sigma_{d-1} * h_{3} \sigma_{d-1} \in \Lambda_{\alpha}\left(\mathbb{R}^{d}\right)$ for some $\alpha>0$. Consequently, if $n \geqslant 3$ and $\left\{h_{j}\right\}_{j=1}^{n} \subset \operatorname{Lip}\left(\mathbb{S}^{d-1}\right)$, then $h_{1} \sigma_{d-1} * \cdots * h_{n} \sigma_{d-1} \in \Lambda_{\alpha}\left(\mathbb{R}^{d}\right)$ for some $\alpha>0$.

\subsection{The case $(d, n)=(2,4)$}

In the course of this subsection only, we shall simplify the notation by writing $\mathrm{d} \sigma=\mathrm{d} \sigma_{1}$. Our goal is to establish a Hölder-type estimate for the fourfold convolution $h_{1} \sigma * h_{2} \sigma * h_{3} \sigma * h_{4} \sigma$, where $\left\{h_{j}\right\}_{j=1}^{4}$ are Lipschitz functions on the unit circle $\mathbb{S}^{1}$. We start with some preparatory work. As in Subsection 4.1, let

$$
\begin{aligned}
& u_{12}(x)=\frac{1}{2}\left(h_{1}\left(x_{1}\right) h_{2}\left(x_{2}\right)+h_{1}\left(x_{2}\right) h_{2}\left(x_{1}\right)\right) \mathbb{1}_{B_{2}}(x), \\
& u_{34}(x)=\frac{1}{2}\left(h_{3}\left(x_{1}\right) h_{4}\left(x_{2}\right)+h_{3}\left(x_{2}\right) h_{4}\left(x_{1}\right)\right) \mathbb{1}_{B_{2}}(x),
\end{aligned}
$$

both of which satisfy the conclusion of Lemma 4.1. For brevity, we write

$$
F(x):=(\sigma * \sigma)(x)=4|x|^{-1}\left(4-|x|^{2}\right)^{-1 / 2} \mathbb{1}_{B_{2}}(x),
$$

as in (4.1), with $d=2$. We will make repeated use of the upper bound

$$
\frac{1}{|x| \sqrt{4-|x|^{2}}}=\frac{\sqrt{4-|x|^{2}}}{4|x|}+\frac{|x|}{4 \sqrt{4-|x|^{2}}} \leqslant \frac{1}{|x|}+\frac{1}{\sqrt{2-|x|}}, \text { for all }|x| \leqslant 2
$$


together with the estimate

$$
\sigma^{* 4}(x) \lesssim(1+|\log | x||) \mathbb{1}_{B_{4}}(x), \text { for all } x \in \mathbb{R}^{2} .
$$

Inequality (4.16) follows from [24, Eq. (3.21)] and, in particular, implies that

$$
|\cdot|{ }^{\beta} \sigma^{* 4} \in L^{\infty}\left(\mathbb{R}^{2}\right) \text {, for every } \beta>0 .
$$

Setting $H_{\gamma}(x)=|x|^{\gamma}\left(\left(u_{12} F\right) *\left(u_{34} F\right)\right)(x)$, we then have that $H_{\gamma} \in L^{\infty}\left(\mathbb{R}^{2}\right)$, for any $\gamma>0$ and $\left\{h_{j}\right\}_{j=1}^{4} \subset L^{\infty}\left(\mathbb{S}^{1}\right)$. This will be used in Proposition 4.6. The following preparatory result quantifies the smallness of the function $\left(\mathbb{1}_{E}(\sigma * \sigma)\right) *(\sigma * \sigma)$ for certain sets $E \subset \mathbb{R}^{2}$ of small Lebesgue measure.

Lemma 4.5. Set $F=\sigma * \sigma$. Let $x \in B_{4} \subset \mathbb{R}^{2}$. Then, for every $\gamma \in(0,1]$ and $s \in\left(0, \frac{\gamma}{2(\gamma+1)}\right)$, there exists a constant $C_{\gamma, s}<\infty$ such that, for all $\varepsilon \in(0,1)$,

$$
\begin{aligned}
& |x|^{\gamma} \int_{A(x, \varepsilon)} F(y) F(x-y) \mathrm{d} y \leqslant C_{\gamma, s} \varepsilon^{\min \left\{\frac{1}{6}, \frac{\gamma}{2(\gamma+1)}-s\right\}}, \\
& |x|^{\gamma} \int_{B_{2} \cap B(x, \varepsilon)} F(y) F(x-y) \mathrm{d} y \leqslant C_{\gamma, s} \varepsilon^{\min \left\{\frac{1}{2}, \gamma-s\right\}},
\end{aligned}
$$

where $A(x, \varepsilon):=\left\{y \in B_{2}: 2-\varepsilon \leqslant|x-y| \leqslant 2\right\}$.

Before embarking on the proof of Lemma 4.5, we discuss a coordinate system that will prove convenient for the argument. Let $x \in \mathbb{R}^{2}, x \neq 0$ be given. A point $y \in \mathbb{R}^{2}$ is uniquely determined by the pair $(|y|,|x-y|)$, up to reflection with respect to the line spanned by $x$. This gives rise to the so-called (two-center) bipolar coordinates, defined by $(r, s)=(|y|,|x-y|)$; see $[10, \S 2]$ and $[12, \S 2.2]$ for the use of this coordinate system in a related setting. The map $y \mapsto(r, s)=(|y|,|x-y|)$ is a two-to-one map from $\mathbb{R}^{2} \backslash \operatorname{span}\{x\}$ to the region determined by the relations $|r-s|<|x|<r+s$, whose Jacobian is given by

$$
\mathrm{d} y=\frac{2 r s}{\left(|x|^{2}-(r-s)^{2}\right)^{\frac{1}{2}}\left((r+s)^{2}-|x|^{2}\right)^{\frac{1}{2}}} \mathrm{~d} r \mathrm{~d} s .
$$

After the change of variables $a=r-s b=r+s$, the Jacobian becomes

$$
\mathrm{d} y=\frac{(a+b)(b-a)}{4\left(|x|^{2}-a^{2}\right)^{\frac{1}{2}}\left(b^{2}-|x|^{2}\right)^{\frac{1}{2}}} \mathrm{~d} a \mathrm{~d} b
$$

Proof of Lemma 4.5. From (4.17), it follows that the left-hand sides of (4.18), (4.19) define bounded functions of $x$, and therefore $\varepsilon>0$ can be taken as small as needed in the argument below. We may also assume that $x \neq 0$; otherwise, (4.18) and (4.19) are trivial.

Let us start with (4.18). Note that $|A(x, \varepsilon)| \lesssim \varepsilon$ and that if $y \in A(x, \varepsilon)$, then $|x-y| \geqslant 2-\varepsilon>1$. As a consequence, the left-hand side of (4.18) can be bounded as follows:

$$
|x|^{\gamma} \int_{A(x, \varepsilon)} \frac{\mathrm{d} y}{|y| \sqrt{4-|y|^{2}}|x-y| \sqrt{4-|x-y|^{2}}} \lesssim|x|^{\gamma} \int_{A(x, \varepsilon)} \frac{\mathrm{d} y}{|y| \sqrt{4-|y|^{2}} \sqrt{2-|x-y|}} .
$$

We then use the upper bound (4.15),

$$
\frac{1}{|y| \sqrt{4-|y|^{2}}} \leqslant \frac{1}{|y|}+\frac{1}{\sqrt{2-|y|}}, \text { for }|y| \leqslant 2,
$$


and are left to analyse the following integrals:

$$
\phi_{1}(x, \varepsilon):=|x|^{\gamma} \int_{A(x, \varepsilon)} \frac{\mathrm{d} y}{|y| \sqrt{2-|x-y|}}, \quad \phi_{2}(x, \varepsilon):=|x|^{\gamma} \int_{A(x, \varepsilon)} \frac{\mathrm{d} y}{\sqrt{2-|y|} \sqrt{2-|x-y|}} .
$$

Analysis of $\phi_{1}(x, \varepsilon)$. We perform a dyadic decomposition of $A(x, \varepsilon)$ via

$$
A_{j}=\left\{y \in B_{2}: 2-2^{-j} \varepsilon \leqslant|x-y| \leqslant 2-2^{-(j+1)} \varepsilon\right\}, \quad j \in \mathbb{N}_{0},
$$

so that

$$
\phi_{1}(x, \varepsilon)=|x|^{\gamma} \int_{A(x, \varepsilon)} \frac{\mathrm{d} y}{|y| \sqrt{2-|x-y|}} \simeq|x|^{\gamma} \sum_{j=0}^{\infty}\left(2^{-j} \varepsilon\right)^{-1 / 2} \int_{A_{j}} \frac{\mathrm{d} y}{|y|} .
$$

Further, consider $\delta \in\left(0, \frac{1}{2}\right)$ and decompose $A_{j}=A_{j, 1} \cup A_{j, 2}$, where $A_{j, 1}=\left\{y \in A_{j}:|y|>\right.$ $\left.\left(2^{-j} \varepsilon\right)^{1 / 2-\delta}\right\}$ and $A_{j, 2}=\left\{y \in A_{j}:|y| \leqslant\left(2^{-j} \varepsilon\right)^{1 / 2-\delta}\right\}=A_{j} \cap B_{\left(2^{-j} \varepsilon\right)^{1 / 2-\delta}}$. Then the contribution of $\left\{A_{j, 1}\right\}_{j \geqslant 0}$ to $\phi_{1}(x, \varepsilon)$ can be bounded as follows:

$$
|x|^{\gamma} \sum_{j=0}^{\infty}\left(2^{-j} \varepsilon\right)^{-1 / 2} \int_{A_{j, 1}} \frac{\mathrm{d} y}{|y|} \lesssim \sum_{j=0}^{\infty}\left(2^{-j} \varepsilon\right)^{-1+\delta}\left|A_{j, 1}\right| \lesssim \sum_{j=0}^{\infty}\left(2^{-j} \varepsilon\right)^{-1+\delta} 2^{-j} \varepsilon \lesssim_{\delta} \varepsilon^{\delta},
$$

where we used that $\left|A_{j, 1}\right| \leqslant\left|A_{j}\right| \lesssim 2^{-j} \varepsilon$. We now proceed to bound the contribution of the sets $A_{j, 2}$ with the help of bipolar coordinates. We have

$$
\begin{aligned}
& \int_{A_{j, 2}} \frac{\mathrm{d} y}{|y|} \simeq \int_{\substack{|r-s|<|x|<r+s \\
0 \leqslant r \leqslant\left(2^{-j} \varepsilon\right)^{1 / 2-\delta} \\
2-2^{-j} \varepsilon \leqslant s \leqslant 2-2^{-(j+1)}}} \frac{s \mathrm{~d} r \mathrm{~d} s}{\sqrt{|x|^{2}-(r-s)^{2}} \sqrt{(r+s)^{2}-|x|^{2}}} \\
& \simeq \int_{\substack{|a|<|x|<b \\
0 \leqslant \frac{a+b}{2} \leqslant\left(2^{-j} \varepsilon\right)^{1 / 2-\delta}}} \frac{\mathrm{d} a \mathrm{~d} b}{\sqrt{|x|^{2}-a^{2}} \sqrt{b^{2}-|x|^{2}}} \\
& 2-2^{-j} \varepsilon \leqslant \frac{b-a}{2} \leqslant 2-2^{-(j+1)} \varepsilon \\
& \simeq \int_{\substack{|a|<|x|<b \\
0 \leqslant \frac{a+b}{2} \leqslant\left(2^{-j} \varepsilon\right)^{1 / 2-\delta}}} \frac{\mathrm{d} a \mathrm{~d} b}{\sqrt{|x|-|a|} \sqrt{b-|x|}} \\
& 2-2^{-j} \varepsilon \leqslant \frac{b-a}{2} \leqslant 2-2^{-(j+1)} \varepsilon \\
& \simeq \int_{-2+2^{-(j+1)}}^{|a|<\leqslant a \leqslant-2+2^{-j} \varepsilon+\left(2^{-j} \varepsilon\right)^{1 / 2-\delta}} \int_{\begin{array}{c}
b>b \leqslant-a+2\left(2^{-j} \varepsilon\right)^{1 / 2-\delta} \\
a+4-2^{-j+1} \varepsilon \leqslant b \leqslant a+4-2^{-j} \varepsilon
\end{array}} \frac{\mathrm{d} a \mathrm{~d} b}{\sqrt{|x|-|a|} \sqrt{b-|x|}} \\
& \lesssim \max \left\{\left(2^{-j} \varepsilon\right)^{1 / 2},\left(2^{-j} \varepsilon\right)^{1 / 4-\delta / 2}\right\} \min \left\{\left(2^{-j} \varepsilon\right)^{1 / 2},\left(2^{-j} \varepsilon\right)^{1 / 4-\delta / 2}\right\} \\
& =\left(2^{-j} \varepsilon\right)^{1 / 2}\left(2^{-j} \varepsilon\right)^{1 / 4-\delta / 2} \text {, }
\end{aligned}
$$

where we used that in the domain of integration $|a| \simeq b \simeq 1$, so that $\sqrt{b+|x|} \simeq 1$ and $\sqrt{|x|+|a|} \simeq 1$. Therefore, the contribution of $\left\{A_{j, 2}\right\}_{j \geqslant 0}$ to $\phi_{1}(x, \varepsilon)$ can be bounded as follows:

$$
\begin{aligned}
|x|^{\gamma} \sum_{j=0}^{\infty}\left(2^{-j} \varepsilon\right)^{-1 / 2} \int_{A_{j, 2}} \frac{\mathrm{d} y}{|y|} & \lesssim \sum_{j \geqslant 0}\left(2^{-j} \varepsilon\right)^{-1 / 2}\left(2^{-j} \varepsilon\right)^{1 / 2}\left(2^{-j} \varepsilon\right)^{1 / 4-\delta / 2} \\
& =\sum_{j \geqslant 0}\left(2^{-j} \varepsilon\right)^{1 / 4-\delta / 2} \simeq_{\delta} \varepsilon^{1 / 4-\delta / 2}
\end{aligned}
$$

Taking $\delta=\frac{1}{6}$, we conclude from (4.24) and (4.25) that $\phi_{1}(x, \varepsilon) \lesssim \varepsilon^{1 / 6}$, which is an acceptable contribution, in the sense that it is smaller than a multiple of the right-hand side of (4.18). 
Analysis of $\phi_{2}(x, \varepsilon)$. The contribution of the region $A^{\prime}:=\left\{y \in A(x, \varepsilon): \sqrt{2-|y|} \geqslant \varepsilon^{\delta}\right\}$ to $\phi_{2}(x, \varepsilon)$ can be estimated as follows:

$$
\begin{aligned}
|x|^{\gamma} \int_{A^{\prime}} \frac{\mathrm{d} y}{\sqrt{2-|y|} \sqrt{2-|x-y|}} & \leqslant|x|^{\gamma} \varepsilon^{-\delta} \int_{A^{\prime}} \frac{\mathrm{d} y}{\sqrt{2-|x-y|}} \lesssim \varepsilon^{-\delta} \int_{A(x, \varepsilon)} \frac{\mathrm{d} y}{\sqrt{4-|x-y|^{2}}} \\
& =\varepsilon^{-\delta} \int_{0}^{2 \pi} \int_{2-\varepsilon}^{2} \frac{r}{\sqrt{4-r^{2}}} \mathrm{~d} r \mathrm{~d} \theta \lesssim \varepsilon^{\frac{1}{2}-\delta} .
\end{aligned}
$$

If $y \in A^{\prime \prime}:=A(x, \varepsilon) \backslash A^{\prime}$, then $2-\varepsilon^{2 \delta} \leqslant|y| \leqslant 2$ and $2-\varepsilon \leqslant|x-y| \leqslant 2$. Therefore, $A^{\prime \prime}$ is contained in the intersection of two annuli of small thickness and located at distance comparable to 2 from the origin. We may further assume that $|x| \geqslant \varepsilon^{\delta}$, because otherwise, given any $s \in(0, \gamma)$,

$$
\phi_{2}(x, \varepsilon) \leqslant\left(\varepsilon^{\delta}\right)^{(\gamma-s)}|x|^{s} \sigma^{* 4}(x) \lesssim_{s} \varepsilon^{(\gamma-s) \delta}
$$

so that $\phi_{2}(x, \varepsilon)=O_{\alpha}\left(\varepsilon^{\alpha}\right)$ for every $\alpha \in(0, \gamma \delta)$. We now apply the same dyadic decomposition of $A(x, \varepsilon)$ as in (4.23) together with a similar one on the second annulus,

$$
D_{k}=\left\{y \in B_{2}: 2-2^{-k} \varepsilon^{2 \delta} \leqslant|y|<2-2^{-(k+1)} \varepsilon^{2 \delta}\right\}, k \in \mathbb{N}_{0},
$$

so that $A^{\prime \prime}=\cup_{j, k \geqslant 0} A_{j} \cap D_{k}$. This yields

$$
|x|^{\gamma} \int_{A^{\prime \prime}} \frac{1}{\sqrt{2-|y|}} \frac{1}{\sqrt{2-|x-y|}} \mathrm{d} y \lesssim|x|^{\gamma} \varepsilon^{-1 / 2-\delta} \sum_{j, k \geqslant 0} 2^{(j+k) / 2}\left|A_{j} \cap D_{k}\right| .
$$

We now use bipolar coordinates to bound $\left|A_{j} \cap D_{k}\right|$. First consider the case where, in addition to $|x| \geqslant \varepsilon^{\delta}$, we have $|x| \leqslant 4-\varepsilon^{\delta}$, so that $A_{j}$ and $D_{k}$ intersect transversely; the intersection consists of two connected components that are symmetric with respect to the line spanned by $x$. Using bipolar coordinates, we have that

$$
\begin{aligned}
& \left|A_{j} \cap D_{k}\right| \simeq \int_{\substack{|r-s|<|x|<r+s \\
2-2^{-k} \varepsilon^{2 \delta} \leqslant r \leqslant 2-2^{-(k+1)} \\
2-2^{-j} \varepsilon \leqslant s \leqslant 2-2^{-(j+1)}}} \frac{r s \mathrm{~d} r}{\varepsilon^{2 \delta}} \\
& \simeq \int_{2-2^{-k} \varepsilon^{2 \delta \leqslant \frac{a+b}{2} \leqslant 2-2^{-(k+1)}} \varepsilon^{2 \delta}}^{|a|<|x|<b} \frac{\mathrm{d} a \mathrm{~d} b}{\sqrt{|x|^{2}-a^{2}} \sqrt{b^{2}-|x|^{2}}} . \\
& 2-2^{-j} \varepsilon \leqslant \frac{b-a}{2} \leqslant 2-2^{-(j+1)} \varepsilon
\end{aligned}
$$

Given $(a, b)$ in the domain of integration from (4.29), it holds that $0 \leqslant 4-b \leqslant \varepsilon^{2 \delta}$ and $|a| \leqslant \varepsilon^{2 \delta}$, so that under the working assumption $|x| \geqslant \varepsilon^{\delta}$, we have $|x|-|a| \gtrsim_{\delta}|x|$ and $|x|+|a| \simeq|x|$. If, in addition, $|x| \leqslant 4-\varepsilon^{\delta}$, then $b-|x| \gtrsim_{\delta} \varepsilon^{\delta}$, and therefore (4.29) yields

$$
\left|A_{j} \cap D_{k}\right| \lesssim \delta|x|^{-1} \varepsilon^{-\delta}\left(2^{-j} \varepsilon\right)\left(2^{-k} \varepsilon^{2 \delta}\right)=|x|^{-1} 2^{-(j+k)} \varepsilon^{1+\delta},
$$

and (4.28) can be bounded as follows:

$$
|x|^{\gamma} \int_{A^{\prime \prime}} \frac{1}{\sqrt{2-|y|}} \frac{1}{\sqrt{2-|x-y|}} \mathrm{d} y \lesssim \delta|x|^{-(1-\gamma)} \sum_{j, k \geqslant 0} 2^{-(j+k) / 2} \varepsilon^{1 / 2} \lesssim \varepsilon^{\frac{1}{2}-\delta(1-\gamma)} .
$$

In the complementary case when $4-\varepsilon^{\delta} \leqslant|x| \leqslant 4$, we have $|x|-|a| \simeq 1$ and $b+|x| \simeq 1$ in the domain 
of integration from (4.29), so that

$$
\begin{aligned}
& \left|A_{j} \cap D_{k}\right| \simeq \int_{2-2^{-k} \varepsilon^{2 \delta \leqslant \frac{a+b}{2} \leqslant 2-2^{-(k+1)}}|a|<|x|<b} \frac{\mathrm{d} a \mathrm{~d} b}{\varepsilon^{2 \delta}} \\
& 2-2^{-j} \varepsilon \leqslant \frac{b-a}{2} \leqslant 2-2^{-(j+1)} \varepsilon
\end{aligned}
$$

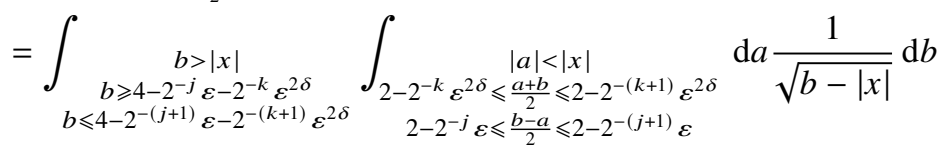

$$
\begin{aligned}
& \lesssim \min \left\{2^{-j} \varepsilon, 2^{-k} \varepsilon^{2 \delta}\right\} \int_{\max \left\{4-2^{-j} \varepsilon-2^{-k} \varepsilon^{2 \delta},|x|\right\}}^{4-2^{-(j+1)} \varepsilon-2^{-(k+1)} \varepsilon^{2 \delta}} \frac{\mathrm{d} b}{\sqrt{b-|x|}} \\
& \lesssim \min \left\{2^{-j} \varepsilon, 2^{-k} \varepsilon^{2 \delta}\right\} \max \left\{\left(2^{-j} \varepsilon\right)^{1 / 2}, 2^{-k / 2} \varepsilon^{\delta}\right\} \\
& =2^{-(j+k) / 2} \varepsilon^{1 / 2+\delta} \min \left\{2^{-j / 2} \varepsilon^{1 / 2}, 2^{-k / 2} \varepsilon^{\delta}\right\} \text {. }
\end{aligned}
$$

In this way, (4.28) is bounded as follows:

$$
\begin{aligned}
|x|^{\gamma} \int_{A^{\prime \prime}} \frac{1}{\sqrt{2-|y|}} \frac{1}{\sqrt{2-|x-y|}} \mathrm{d} y & \lesssim|x|^{\gamma} \sum_{j, k \geqslant 0} \min \left\{2^{-j / 2} \varepsilon^{1 / 2}, 2^{-k / 2} \varepsilon^{\delta}\right\} \\
& \lesssim \sum_{j \geqslant 0}\left(2^{-j} \varepsilon\right)^{1 / 2}+\sum_{j \geqslant 0}\left(2^{-j} \varepsilon\right)^{1 / 2}\left|\log \left(2^{-j} \varepsilon^{1-2 \delta}\right)\right| \\
& \lesssim_{s} \sum_{j \geqslant 0}\left(2^{-j} \varepsilon\right)^{1 / 2}+\sum_{j \geqslant 0} \varepsilon^{\delta}\left(2^{-j} \varepsilon^{1-2 \delta}\right)^{1 / 2-s} \\
& \lesssim_{s} \varepsilon^{\frac{1}{2}-s}
\end{aligned}
$$

for any $s \in\left(0, \frac{1}{2}\right)$. In the passage from the first line to the second line above, we split the sum in $k$ according to the partition $\mathbb{N}_{0}=\left\{k \in \mathbb{N}_{0}: k \geqslant\left|\log _{2}\left(2^{-j} \varepsilon^{1-2 \delta}\right)\right|\right\} \cup\left\{k \in \mathbb{N}_{0}: k<\left|\log _{2}\left(2^{-j} \varepsilon^{1-2 \delta}\right)\right|\right\}=$ : $E_{1} \cup E_{2}$, respectively, where $\log _{2}(\cdot)$ denotes the base 2 logarithm. On $E_{1}$, the sum is a convergent geometric series whose value is proportional to the first term; hence it is $\lesssim\left(2^{-j} \varepsilon\right)^{1 / 2}$. On $E_{2}$, the summands are constant, and the contribution to the sum equals $\left(2^{-j} \varepsilon\right)^{1 / 2}\left|E_{2}\right| \simeq\left(2^{-j} \varepsilon\right)^{1 / 2}\left|\log \left(2^{-j} \varepsilon^{1-2 \delta}\right)\right|$. As a result of (4.26), (4.27), (4.30) and (4.31), we conclude the upper bound $\phi_{2}(x, \varepsilon) \lesssim \delta, s \max \left\{\varepsilon^{\frac{1}{2}-\delta}, \varepsilon^{(\gamma-s) \delta}\right\}$ for every $\delta \in\left(0, \frac{1}{2}\right)$ and $s \in(0, \gamma)$. Optimising in $\delta$, we are thus led to the estimate $\phi_{2}(x, \varepsilon) \lesssim_{s} \varepsilon^{\frac{\gamma}{2(\gamma+1)}-s}$ for every $s>0$. This concludes the verification of (4.18).

To handle (4.19), start by noting that

$$
|x|^{\gamma} \int_{B_{2} \cap B(x, \varepsilon)} F(y) F(x-y) \mathrm{d} y=16|x|^{\gamma} \int_{B_{2} \cap B(x, \varepsilon)} \frac{1}{|y| \sqrt{4-|y|^{2}}} \frac{1}{|x-y| \sqrt{4-|x-y|^{2}}} \mathrm{~d} y .
$$

Because $\varepsilon<1$, we may remove the term $\sqrt{4-|x-y|^{2}}$ from the latter integrand at the expense of a universal constant. After an application of (4.22), we are then left to study the following integrals:

$$
\phi_{3}(x, \varepsilon):=|x|^{\gamma} \int_{B_{2} \cap B(x, \varepsilon)} \frac{\mathrm{d} y}{|y||x-y|}, \quad \phi_{4}(x, \varepsilon):=|x|^{\gamma} \int_{B_{2} \cap B(x, \varepsilon)} \frac{\mathrm{d} y}{\sqrt{2-|y|}|x-y|} .
$$

Analysis of $\phi_{3}(x, \varepsilon)$. Decompose the region of integration $B_{2} \cap B(x, \varepsilon)=A_{1} \cup A_{2}$, where

$$
\begin{aligned}
& A_{1}:=B(x, \varepsilon) \cap\left\{y \in B_{2}:|y| \geqslant \varepsilon^{1 / 2}\right\}, \\
& A_{2}:=B(x, \varepsilon) \cap\left\{y \in B_{2}:|y|<\varepsilon^{1 / 2}\right\} .
\end{aligned}
$$


On the region $A_{1}$, we may simply estimate

$$
|x|^{\gamma} \int_{A_{1}} \frac{\mathrm{d} y}{|y||x-y|} \leqslant|x|^{\gamma} \varepsilon^{-1 / 2} \int_{B(x, \varepsilon)} \frac{\mathrm{d} y}{|x-y|}=2 \pi|x|^{\gamma} \varepsilon^{-1 / 2} \varepsilon \lesssim \varepsilon^{1 / 2} .
$$

We further split $A_{2}=A_{2}^{\prime} \cup A_{2}^{\prime \prime}$, with

$$
A_{2}^{\prime}:=A_{2} \cap\{y:|y| \geqslant|x-y|\}, \text { and } A_{2}^{\prime \prime}:=A_{2} \cap\{y:|y|<|x-y|\} .
$$

If $y \in A_{2}^{\prime}$, then $|y| \geqslant \frac{1}{2}|x|$, and therefore

$$
|x|^{\gamma} \int_{A_{2}^{\prime}} \frac{\mathrm{d} y}{|y||x-y|} \lesssim \int_{A_{2}^{\prime}} \frac{\mathrm{d} y}{|y|^{1-\gamma}|x-y|} .
$$

Now, $|y|^{-(1-\gamma)} \mathbb{1}_{B_{2}} \in L^{p}\left(\mathbb{R}^{2}\right)$ for every $1 \leqslant p<\frac{2}{1-\gamma}$, and $|y-x|^{-1} \mathbb{1}_{B_{2}} \in L^{q}\left(\mathbb{R}^{2}\right)$ for every $1 \leqslant q<2$. Taking $2<p<\frac{2}{1-\gamma}$, its conjugate satisfies $\frac{2}{1+\gamma}<p^{\prime}<2$, and so by Hölder's inequality we have that

$$
\begin{aligned}
\int_{A_{2}^{\prime}} \frac{\mathrm{d} y}{|y|^{1-\gamma}|x-y|} & \leqslant\left(\int_{B_{\varepsilon^{1 / 2}}} \frac{\mathrm{d} y}{|y|^{p(1-\gamma)}}\right)^{\frac{1}{p}}\left(\int_{B(x, \varepsilon)} \frac{\mathrm{d} y}{|x-y|^{p^{\prime}}}\right)^{\frac{1}{p^{\prime}}} \\
& =\frac{2 \pi}{(2-p(1-\gamma))^{\frac{1}{p}}\left(2-p^{\prime}\right)^{\frac{1}{p^{\prime}}}} \varepsilon^{\frac{p(1+\gamma)-2}{2 p}} .
\end{aligned}
$$

Note that $(p(1+\gamma)-2) /(2 p)$ strictly increases to $\gamma$ as $p$ increases to $2 /(1-\gamma)$. In this way, we obtain

$$
|x|^{\gamma} \int_{A_{2}^{\prime}} \frac{\mathrm{d} y}{|y||x-y|} \lesssim_{s} \varepsilon^{\gamma-s}
$$

for every $s \in(0, \gamma)$. If $y \in A_{2}^{\prime \prime}$, then $|x-y| \geqslant \frac{1}{2}|x|$ and $|y|<|x-y| \leqslant \varepsilon$; in particular, $A_{2}^{\prime \prime} \subset B_{\varepsilon}$. Therefore, if $2<p<\frac{2}{1-\gamma}$, then

$$
\begin{aligned}
|x|^{\gamma} \int_{A_{2}^{\prime \prime}} \frac{\mathrm{d} y}{|y||x-y|} & \lesssim \int_{A_{2}^{\prime \prime}} \frac{\mathrm{d} y}{|y||x-y|^{1-\gamma}} \leqslant\left(\int_{B_{\varepsilon}} \frac{\mathrm{d} y}{|y|^{p^{\prime}}}\right)^{\frac{1}{p^{\prime}}}\left(\int_{B(x, \varepsilon)} \frac{\mathrm{d} y}{|x-y|^{p(1-\gamma)}}\right)^{\frac{1}{p}} \\
& \lesssim \varepsilon^{\frac{2-p^{\prime}}{p^{\prime}}} \varepsilon^{\frac{2-p(1-\gamma)}{p}}=\varepsilon^{\gamma} .
\end{aligned}
$$

We conclude that $\phi_{3}(x, \varepsilon) \lesssim_{s} \varepsilon^{\min \left\{\frac{1}{2}, \gamma-s\right\}}$ for every $s \in(0, \gamma)$.

Analysis of $\phi_{4}(x, \varepsilon)$. Proceeding as before, we decompose the region of integration $B_{2} \cap B(x, \varepsilon)=$ $D_{1} \cup D_{2}$, where

$$
\begin{aligned}
& D_{1}:=B(x, \varepsilon) \cap\left\{y \in B_{2}: \sqrt{2-|y|} \geqslant \varepsilon^{1 / 2}\right\}, \\
& D_{2}:=B(x, \varepsilon) \cap\left\{y \in B_{2}: \sqrt{2-|y|}<\varepsilon^{1 / 2}\right\} .
\end{aligned}
$$

On the region $D_{1}$, we may simply estimate

$$
|x|^{\gamma} \int_{D_{1}} \frac{\mathrm{d} y}{\sqrt{2-|y|}|x-y|} \leqslant|x|^{\gamma} \varepsilon^{-1 / 2} \int_{B(x, \varepsilon)} \frac{\mathrm{d} y}{|x-y|} \lesssim \varepsilon^{1 / 2} .
$$

If $y \in D_{2}$, then $2-\varepsilon<|y| \leqslant 2$, and so $2-2 \varepsilon \leqslant|x| \leqslant 2+\varepsilon$. We may apply a dyadic decomposition,

$$
V_{j}=\left\{y \in D_{2}: 2^{-(j+1)} \varepsilon \leqslant|x-y| \leqslant 2^{-j} \varepsilon\right\}, j \in \mathbb{N}_{0},
$$


so that, letting $P\left(V_{j}\right)$ denote the image of $V_{j}$ under the polar coordinate map, and further writing $P\left(V_{j}\right)=\{(r, \theta): \theta \in \Theta, r \in R(\theta)\}$ for some $\Theta \subseteq[0,2 \pi)$ and $R(\theta) \subseteq[0, \infty)$, we have that

$$
\begin{gathered}
|x|^{\gamma} \int_{D_{2}} \frac{\mathrm{d} y}{\sqrt{2-|y|}|x-y|} \lesssim|x|^{\gamma} \sum_{j=0}^{\infty} 2^{j} \varepsilon^{-1} \int_{V_{j}} \frac{\mathrm{d} y}{\sqrt{4-|y|^{2}}}=|x|^{\gamma} \sum_{j=0}^{\infty} 2^{j} \varepsilon^{-1} \int_{P\left(V_{j}\right)} \frac{r \mathrm{~d} r \mathrm{~d} \theta}{\sqrt{4-r^{2}}} \\
\lesssim|x|^{\gamma} \sum_{j=0}^{\infty} 2^{j} \varepsilon^{-1} \int_{\Theta}|R(\theta)|^{1 / 2} \mathrm{~d} \theta \lesssim|x|^{\gamma} \sum_{j=0}^{\infty}\left(2^{-j} \varepsilon\right)^{-1}\left(2^{-j} \varepsilon\right)^{1 / 2}\left(2^{-j} \varepsilon\right) \lesssim \varepsilon^{1 / 2} .
\end{gathered}
$$

In the second-to-last inequality, we used the fact that the length of the intersection of any line with the annulus $V_{j}$ is $O\left(2^{-j} \varepsilon\right)$ (so that $|R(\theta)|^{1 / 2} \lesssim\left(2^{-j} \varepsilon\right)^{1 / 2}$ ), whereas the angular span $\Theta$ has measure $O\left(2^{-j} \varepsilon\right)$ given that $|x| \gtrsim 1$ and $V_{j} \subseteq B\left(x, 2^{-j} \varepsilon\right)$. We conclude that $\phi_{4}(x, \varepsilon) \lesssim \varepsilon^{1 / 2}$, and therefore (4.19) is verified. This finishes the proof of the lemma.

Proposition 4.6. Given $\gamma>0$ and $\left\{h_{j}\right\}_{j=1}^{4} \subset \operatorname{Lip}\left(\mathbb{S}^{1}\right)$, let $H_{\gamma}=|\cdot|^{\gamma}\left(h_{1} \sigma * h_{2} \sigma * h_{3} \sigma * h_{4} \sigma\right)$. Then there exist $\tau>0$ and $C<\infty$ such that, for every $x, x^{\prime} \in \mathbb{R}^{2}$,

$$
\left|H_{\gamma}(x)-H_{\gamma}\left(x^{\prime}\right)\right| \leqslant C\left|x-x^{\prime}\right|^{\tau},
$$

where $C \leqslant C_{0} \prod_{j=1}^{4}\left\|h_{j}\right\|_{\operatorname{Lip}\left(\mathbb{S}^{1}\right)}$ for some constant $C_{0}<\infty$ depending only on $\gamma$.

The proof of Proposition 4.6 will reveal that one can take any $\tau<\min \left\{\frac{1}{14}, \frac{\gamma}{2(3 \gamma+2)}\right\}$. To a large extent, the proof follows similar lines to those of Proposition 4.3, and so at times we shall be brief. The main difference is that now the extra singularity of $(\sigma * \sigma)(x)$ along the boundary circle $|x|=2$ also needs to be accounted for.

Proof of Proposition 4.6. Because the case $\gamma>1$ follows from that of $\gamma \in(0,1]$, the latter condition will be assumed throughout the proof. By homogeneity, we may assume that $\left\|h_{j}\right\|_{\text {Lip }}=1,1 \leqslant j \leqslant 4$. Because $H_{\gamma}$ is compactly supported, it is enough to consider $x, x^{\prime} \in \mathbb{R}^{2}$ satisfying $\left|x-x^{\prime}\right| \ll 1$; we further assume $|x| \leqslant \min \left\{4,\left|x^{\prime}\right|\right\}$. With the notation introduced above (recall (4.12)-(4.14)), we have that

$$
\begin{aligned}
\mid H_{\gamma}(x)- & H_{\gamma}\left(x^{\prime}\right)|=||x|^{\gamma}\left(u_{12} F * u_{34} F\right)(x)-\left|x^{\prime}\right|^{\gamma}\left(u_{12} F * u_{34} F\right)\left(x^{\prime}\right) \mid \\
& \leqslant|x|^{\gamma}\left|\left(u_{12} F * u_{34} F\right)(x)-\left(u_{12} F * u_{34} F\right)\left(x^{\prime}\right)\right|+\left.|| x\right|^{\gamma}-\left|x^{\prime}\right|^{\gamma}||\left(u_{12} F * u_{34} F\right)\left(x^{\prime}\right) \mid .
\end{aligned}
$$

The second summand in (4.34) satisfies the upper bound

$$
\begin{aligned}
\|\left. x\right|^{\gamma}-\left|x^{\prime}\right|^{\gamma}||\left(u_{12} F * u_{34} F\right)\left(x^{\prime}\right) \mid & \leqslant\left.|| x\right|^{\gamma}-\left|x^{\prime}\right|^{\gamma}\left|\sigma^{* 4}\left(x^{\prime}\right) \lesssim_{\gamma}\right||x|-\left.\left|x^{\prime}\right|\right|^{\gamma} \sigma^{* 4}\left(x^{\prime}\right) \\
& \leqslant\left|x-x^{\prime}\right|^{s}\left|x^{\prime}\right|^{\gamma-s} \sigma^{* 4}\left(x^{\prime}\right) \\
& \lesssim \gamma, s\left|x-x^{\prime}\right|^{s}
\end{aligned}
$$

for any $s \in(0, \gamma)$, where in the third inequality we used $|x| \leqslant\left|x^{\prime}\right|$ to obtain ||$x|-| x^{\prime}|| \leqslant\left|x^{\prime}\right|$ and in the last inequality we invoked (4.17). The first summand in (4.34) can be rewritten as the sum of two integrals,

$$
\begin{aligned}
|x|^{\gamma}\left(\left(u_{12} F * u_{34} F\right)(x)-\left(u_{12} F * u_{34} F\right)\left(x^{\prime}\right)\right) & \\
=|x|^{\gamma} \int_{B_{2}} u_{12}(y) F(y) F\left(x^{\prime}-y\right)\left(u_{34}(x-y)-u_{34}\left(x^{\prime}-y\right)\right) \mathrm{d} y & +|x|^{\gamma} \int_{B_{2}} u_{12}(y) F(y)\left(F(x-y)-F\left(x^{\prime}-y\right)\right) u_{34}(x-y) \mathrm{d} y .
\end{aligned}
$$


We denote the integrals on the right-hand side of the latter identity by $I$ and $I I$, respectively, and proceed to estimate them separately.

Estimating $I$. The first step is to restrict the domain of integration to the region where $x-y, x^{\prime}-y \in B_{2}$, plus a $O\left(\left|x-x^{\prime}\right|^{\alpha}\right)$ remainder, for some $\alpha>0$ to be determined. With this purpose in mind, decompose $B_{2}=U \cup U^{\prime} \cup V \cup W$, where

$$
\begin{aligned}
U:= & \left\{y \in B_{2}:\left|x^{\prime}-y\right|<2 \leqslant|x-y|\right\}, U^{\prime}:=\left\{y \in B_{2}:|x-y|<2 \leqslant\left|x^{\prime}-y\right|\right\}, \\
& V:=\left\{y \in B_{2}:|x-y|,\left|x^{\prime}-y\right|<2\right\}, W:=\left\{y \in B_{2}: 2 \leqslant\left|x^{\prime}-y\right|,|x-y|\right\} .
\end{aligned}
$$

The integrand of $I$ vanishes on $U^{\prime} \cup W$, and so we are left to analyse the integrals over the regions $U$ and $V$. As in (4.5), we have that

$$
U \subseteq\left\{y \in B_{2}: 2-\left|x-x^{\prime}\right| \leqslant\left|x^{\prime}-y\right| \leqslant 2\right\}=: A\left(x^{\prime},\left|x-x^{\prime}\right|\right)
$$

and, therefore,

$$
\begin{aligned}
|x|^{\gamma} \int_{U}\left|u_{12}(y) u_{34}\left(x^{\prime}-y\right)\right| F(y) F\left(x^{\prime}-y\right) \mathrm{d} y & \leqslant|x|^{\gamma} \int_{A\left(x^{\prime},\left|x-x^{\prime}\right|\right)} F(y) F\left(x^{\prime}-y\right) \mathrm{d} y \\
& \lesssim \gamma, s\left|x-x^{\prime}\right|^{\min \left\{\frac{1}{6}, \frac{\gamma}{2(\gamma+1)}-s\right\}},
\end{aligned}
$$

for every $s \in\left(0, \frac{\gamma}{2(\gamma+1)}\right)$, where the latter inequality follows from estimate (4.18). We now consider the integral over the set $V$. To begin with, note that Lemma 4.1 implies the pointwise estimate

$$
\left|u_{34}(x-y)-u_{34}\left(x^{\prime}-y\right)\right| \lesssim\left|x-x^{\prime}\right|^{1 / 2}+\left|\frac{x-y}{|x-y|}-\frac{x^{\prime}-y}{\left|x^{\prime}-y\right|}\right|,
$$

provided that $x-y, x^{\prime}-y \in B_{2}$. The contribution of the region

$$
R:=\left\{y \in V:\left|\frac{x-y}{|x-y|}-\frac{x^{\prime}-y}{\left|x^{\prime}-y\right|}\right| \leqslant\left|x-x^{\prime}\right|^{1 / 2}\right\}
$$

to the integral $I$ is easy to estimate. In view of (4.37) and (4.38), because $|x| \leqslant\left|x^{\prime}\right|$,

$$
\begin{aligned}
|x|^{\gamma} \mid \int_{R} u_{12}(y) F(y) F\left(x^{\prime}-y\right) & \left(u_{34}(x-y)-u_{34}\left(x^{\prime}-y\right)\right) \mathrm{d} y \mid \\
& \lesssim\left|x^{\prime}\right|^{\gamma}\left(\int_{R} F(y) F\left(x^{\prime}-y\right) \mathrm{d} y\right)\left|x-x^{\prime}\right|^{1 / 2} \\
& \leqslant\left|x^{\prime}\right|^{\gamma} \sigma_{2}^{* 4}\left(x^{\prime}\right)\left|x-x^{\prime}\right|^{1 / 2} \lesssim \gamma\left|x-x^{\prime}\right|^{1 / 2},
\end{aligned}
$$

where in the latter inequality we invoked (4.17). If $y \in V \backslash R$, then $\left|x^{\prime}-y\right| \leqslant 2\left|x-x^{\prime}\right|^{1 / 2}$ as in (4.9). The contribution of the region $V \backslash R$ can then be estimated as follows:

$$
\begin{aligned}
|x|^{\gamma} \mid \int_{V \backslash R} u_{12}(y) F(y) & F\left(x^{\prime}-y\right)\left(u_{34}(x-y)-u_{34}\left(x^{\prime}-y\right)\right) \mathrm{d} y \mid \\
& \lesssim|x|^{\gamma} \int_{V \backslash R}\left|u_{12}(y)\right| F(y) F\left(x^{\prime}-y\right)\left|\frac{x-y}{|x-y|}-\frac{x^{\prime}-y}{\left|x^{\prime}-y\right|}\right| \mathrm{d} y \\
& \leqslant 2|x|^{\gamma} \int_{V \cap B\left(x^{\prime}, 2\left|x-x^{\prime}\right|^{1 / 2}\right)} F(y) F\left(x^{\prime}-y\right) \mathrm{d} y \\
& \lesssim \gamma, s\left|x-x^{\prime}\right|^{\min \left\{\frac{1}{4}, \frac{\gamma}{2}-s\right\}},
\end{aligned}
$$

for every $s \in\left(0, \frac{\gamma}{2}\right)$. The latter inequality is a consequence of estimate (4.19). 
Estimating $I I$. The integral $I I$ is bounded in absolute value by

$$
|x|^{\gamma} \int_{B_{2}} F(y)\left|F(x-y)-F\left(x^{\prime}-y\right)\right| \mathrm{d} y .
$$

Decompose $B_{2}=U \cup U^{\prime} \cup V \cup W$ as in (4.35) and note that the integrand of $I I$ vanishes on $W$. The contribution of the region $U \cup U^{\prime}$ can be handled with estimate (4.18) as follows (recall (4.36)):

$$
\begin{aligned}
|x|^{\gamma} \int_{U \cup U^{\prime}} F(y)\left|F(x-y)-F\left(x^{\prime}-y\right)\right| \mathrm{d} y & \leqslant 2|x|^{\gamma} \int_{A\left(x,\left|x-x^{\prime}\right|\right)} F(y) F(x-y) \mathrm{d} y \\
& \lesssim \gamma\left|x-x^{\prime}\right|^{\min \left\{\frac{1}{6}, \frac{\gamma}{2(\gamma+1)}-s\right\}},
\end{aligned}
$$

for every $s \in\left(0, \frac{\gamma}{2(\gamma+1)}\right)$. The estimate on the region $V$ is more delicate, and we split the analysis into two cases. Inside the ball $|x-y| \leqslant\left|x-x^{\prime}\right|^{1 / 4}$, we also have that $\left|x^{\prime}-y\right| \leqslant\left|x-x^{\prime}\right|+|x-y| \lesssim\left|x-x^{\prime}\right|^{1 / 4}$. In order to bound the corresponding piece of $I I$, it suffices to consider the integral

$$
\varphi\left(x, x^{\prime}\right):=\left|x^{\prime}\right|^{\gamma} \int_{V \cap B\left(x^{\prime},\left|x-x^{\prime}\right|^{1 / 4}\right)} F(y) F\left(x^{\prime}-y\right) \mathrm{d} y,
$$

which by (4.19) satisfies $\varphi\left(x, x^{\prime}\right) \lesssim_{s}\left|x-x^{\prime}\right|^{\frac{1}{4} \min \left\{\frac{1}{2}, \gamma-s\right\}}$ for every $s \in(0, \gamma)$. We proceed with the analysis of the complementary region; that is, where $|x-y|>\left|x-x^{\prime}\right|^{1 / 4}$. If $y \in B_{2}$, then

$$
F(y)=\frac{4}{|y| \sqrt{4-|y|^{2}}}=\frac{\sqrt{4-|y|^{2}}}{|y|}+\frac{|y|}{\sqrt{4-|y|^{2}}}
$$

and, as a consequence,

$$
\begin{aligned}
& \left|F(x-y)-F\left(x^{\prime}-y\right)\right| \\
& \leqslant\left|\frac{\sqrt{4-|x-y|^{2}}}{|x-y|}-\frac{\sqrt{4-\left|x^{\prime}-y\right|^{2}}}{\left|x^{\prime}-y\right|}\right|+\left|\frac{|x-y|}{\sqrt{4-|x-y|^{2}}}-\frac{\left|x^{\prime}-y\right|}{\sqrt{4-\left|x^{\prime}-y\right|^{2}}}\right| \\
& \leqslant \sqrt{4-|x-y|^{2}}\left|\frac{1}{|x-y|}-\frac{1}{\left|x^{\prime}-y\right|}\right|+\frac{1}{\left|x^{\prime}-y\right|}\left|\sqrt{4-|x-y|^{2}}-\sqrt{4-\left|x^{\prime}-y\right|^{2}}\right| \\
& \quad+|x-y|\left|\frac{1}{\sqrt{4-|x-y|^{2}}}-\frac{1}{\sqrt{4-\left|x^{\prime}-y\right|^{2}}}\right|+\frac{1}{\sqrt{4-\left|x^{\prime}-y\right|^{2}}}|x-y|-\left|x^{\prime}-y\right| \mid .
\end{aligned}
$$

Using the triangle inequality and recalling that $F\left(x^{\prime}-y\right)=\frac{4}{\left|x^{\prime}-y\right| \sqrt{4-\left|x^{\prime}-y\right|^{2}}}$,

$$
\begin{aligned}
\left|F(x-y)-F\left(x^{\prime}-y\right)\right| \lesssim & \frac{\left|x-x^{\prime}\right|}{|x-y|\left|x^{\prime}-y\right|}+\left|\frac{1}{\sqrt{4-|x-y|^{2}}}-\frac{1}{\sqrt{4-\left|x^{\prime}-y\right|^{2}}}\right| \\
& +\frac{\left|x-x^{\prime}\right|^{1 / 2}}{\left|x^{\prime}-y\right|}+\frac{\left|x-x^{\prime}\right|}{\sqrt{4-\left|x^{\prime}-y\right|^{2}}} \\
\lesssim & \frac{\left|x-x^{\prime}\right|}{|x-y|\left|x^{\prime}-y\right|}+\left|\frac{1}{\sqrt{4-|x-y|^{2}}}-\frac{1}{\sqrt{4-\left|x^{\prime}-y\right|^{2}}}\right| \\
& +\left|x-x^{\prime}\right|^{1 / 2} F\left(x^{\prime}-y\right) .
\end{aligned}
$$


If $|x-y|>\left|x-x^{\prime}\right|^{1 / 4}$, then $\left|x^{\prime}-y\right| \geqslant|x-y|-\left|x-x^{\prime}\right| \gtrsim\left|x-x^{\prime}\right|^{1 / 4}$. Then for $y \in V \cap B\left(x,\left|x-x^{\prime}\right|^{1 / 4}\right)^{C}$ we obtain

$$
\begin{gathered}
\left|F(x-y)-F\left(x^{\prime}-y\right)\right| \lesssim\left|x-x^{\prime}\right|^{1 / 2}+\left|\frac{1}{\sqrt{4-|x-y|^{2}}}-\frac{1}{\sqrt{4-\left|x^{\prime}-y\right|^{2}}}\right| \\
+\left|x-x^{\prime}\right|^{1 / 2} F\left(x^{\prime}-y\right) .
\end{gathered}
$$

It follows that the contribution of this region to the integral $I I$ is bounded by

$$
\begin{aligned}
& |x|^{\gamma}\left|x-x^{\prime}\right|^{1 / 2} \int_{V} F(y) \mathrm{d} y+|x|^{\gamma}\left|x-x^{\prime}\right|^{1 / 2} \int_{V} F(y) F\left(x^{\prime}-y\right) \mathrm{d} y \\
& \quad+|x|^{\gamma} \int_{V \cap B\left(x,\left|x-x^{\prime}\right|^{1 / 4}\right)^{C}} F(y)\left|\frac{1}{\sqrt{4-|x-y|^{2}}}-\frac{1}{\sqrt{4-\left|x^{\prime}-y\right|^{2}}}\right| \mathrm{d} y \\
& \lesssim\left|x-x^{\prime}\right|^{1 / 2}+|x|^{\gamma} \int_{V \cap B\left(x,\left|x-x^{\prime}\right|^{1 / 4}\right)^{C}} F(y)\left|\frac{1}{\sqrt{4-|x-y|^{2}}}-\frac{1}{\sqrt{4-\left|x^{\prime}-y\right|^{2}}}\right| \mathrm{d} y,
\end{aligned}
$$

where we used that $|x| \leqslant\left|x^{\prime}\right|,|x|^{\gamma} \int_{V} F(y) F\left(x^{\prime}-y\right) \mathrm{d} y \leqslant\left|x^{\prime}\right|^{\gamma} \sigma^{* 4}\left(x^{\prime}\right) \leqslant C_{\gamma}<\infty$ and $\int_{V} F(y) \mathrm{d} y \leqslant$ $\sigma\left(\mathbb{S}^{1}\right)^{2}$. The last integral left to analyse is

$$
|x|^{\gamma} \int_{V \cap B\left(x,\left|x-x^{\prime}\right|^{1 / 4}\right) C} F(y)\left|\frac{1}{\sqrt{4-|x-y|^{2}}}-\frac{1}{\sqrt{4-\left|x^{\prime}-y\right|^{2}}}\right| \mathrm{d} y .
$$

Given $\delta \in\left(0, \frac{1}{2}\right)$, we further decompose the domain of integration, $V \cap B\left(x,\left|x-x^{\prime}\right|^{1 / 4}\right)^{C}$, into the subregion where $4-|x-y|^{2} \geqslant\left|x-x^{\prime}\right|^{\delta}$ and its complement. If $y \in V$ satisfies $4-|x-y|^{2} \geqslant\left|x-x^{\prime}\right|^{\delta}$, then $4-\left|x^{\prime}-y\right|^{2} \gtrsim\left|x-x^{\prime}\right|^{\delta}$, and so

$$
\left|\frac{1}{\sqrt{4-|x-y|^{2}}}-\frac{1}{\sqrt{4-\left|x^{\prime}-y\right|^{2}}}\right| \lesssim \frac{\left|x-x^{\prime}\right|^{1 / 2}}{\sqrt{4-|x-y|^{2}} \sqrt{4-\left|x^{\prime}-y\right|^{2}}} \lesssim\left|x-x^{\prime}\right|^{\frac{1}{2}-\delta} .
$$

Therefore, the contribution of this region to the integral (4.39) is bounded by

$$
|x|^{\gamma}\left|x-x^{\prime}\right|^{\frac{1}{2}-\delta} \int_{B_{2}} F(y) \mathrm{d} y \lesssim\left|x-x^{\prime}\right|^{\frac{1}{2}-\delta} .
$$

Finally, if $4-|x-y|^{2}<\left|x-x^{\prime}\right|^{\delta}$, then $2-|x-y| \leqslant \frac{1}{2}\left|x-x^{\prime}\right|^{\delta}$, so that this region is contained in the annular domain

$$
A(x, \varepsilon):=\left\{y \in B_{2}: 2-\varepsilon \leqslant|x-y| \leqslant 2\right\},
$$

for $\varepsilon=\frac{1}{2}\left|x-x^{\prime}\right|^{\delta}$. Because we also have $2-\left|x^{\prime}-y\right| \leqslant\left|x-x^{\prime}\right|^{\delta}$ if $\left|x-x^{\prime}\right| \ll 1$, the region is also contained in $A\left(x^{\prime}, 2 \varepsilon\right)$. The triangle inequality implies that the integral over the latter region is bounded by (two times) the quantity

$$
\tilde{\varphi}\left(x, x^{\prime}\right):=|x|^{\gamma} \int_{A\left(x,\left|x-x^{\prime}\right|^{\delta}\right)} F(y) F(x-y) \mathrm{d} y .
$$

One last application of estimate (4.18) reveals that $\tilde{\varphi}\left(x, x^{\prime}\right) \lesssim \gamma, s\left|x-x^{\prime}\right|^{\delta \min \left\{\frac{1}{6}, \frac{\gamma}{2(\gamma+1)}-s\right\}}$ for every $s \in\left(0, \frac{\gamma}{2(\gamma+1)}\right)$. This concludes the proof of the proposition.

Remark 4.7. More generally, all higher convolutions $G_{n}:=h_{1} \sigma * \cdots * h_{n} \sigma, n \geqslant 5$, are Hölder continuous functions whenever $\left\{h_{j}\right\}_{j=1}^{n} \subset \operatorname{Lip}\left(\mathbb{S}^{1}\right)$. Indeed, this can be verified for the fifth convolution $G_{5}=h_{1} \sigma * \cdots * h_{5} \sigma$ by writing $G_{5}=\left(|\cdot|^{-\gamma} H_{\gamma}\right) * h_{5} \sigma$ for any $\gamma \in(0,1)$, studying the differences 
$\left|G_{5}(x)-G_{5}\left(x^{\prime}\right)\right|$ and using Proposition 4.6 together with the methods employed in its proof. Once it is known that $G_{5} \in \Lambda_{\alpha}\left(\mathbb{R}^{2}\right)$ for some $\alpha>0$, it is immediate that $G_{n} \in \Lambda_{\alpha}\left(\mathbb{R}^{2}\right)$ for every $n \geqslant 5$. This can be improved, for example, by noting that $G_{10} \in \Lambda_{2 \alpha}\left(\mathbb{R}^{2}\right)$.

\section{5. $\mathcal{H}^{s}$-bound for a restricted convolution operator}

Consider a function $H: \mathbb{R}^{d} \rightarrow \mathbb{C}$ supported on the ball $B_{R} \subset \mathbb{R}^{d}$, for some $R>0$, satisfying, for some $\alpha \in(0,1)$ and $C<\infty$,

$$
\left|H(x)-H\left(x^{\prime}\right)\right| \leqslant C\left|x-x^{\prime}\right|^{\alpha}+C\left|\frac{x}{|x|}-\frac{x^{\prime}}{\left|x^{\prime}\right|}\right|, \text { for every } x, x^{\prime} \in B_{R} \backslash\{0\} .
$$

Then $H \in L^{\infty}\left(\mathbb{R}^{d}\right)$ and is continuous in $B_{R} \backslash\{0\}$. Given $\gamma \in[0,1]$, let $K_{\gamma}=|\cdot|^{-\gamma} H$ and define the corresponding linear operator $\mathcal{K}_{\gamma}: C^{0}\left(\mathbb{S}^{d-1}\right) \rightarrow L^{2}\left(\mathbb{S}^{d-1}\right)$ via

$$
\left(\mathcal{K}_{\gamma} f\right)(\omega)=\int_{\mathbb{S}^{d-1}} f(v) K_{\gamma}(\omega-v) \mathrm{d} \sigma_{d-1}(v) .
$$

Lemma 5.1. Let $d \geqslant 3$ and $\gamma \in[0,1]$ or $d=2$ and $\gamma \in[0,1)$. Let $R>0$ and $\mathcal{K}_{\gamma}$ be the linear operator defined in (5.2) above. Then there exists $\delta=\delta(d, \gamma, R)>0$ such that $\mathcal{K}_{\gamma}$ extends to a bounded operator from $L^{2}\left(\mathbb{S}^{d-1}\right)$ to $\mathcal{H}^{\delta}\left(\mathbb{S}^{d-1}\right)$.

Proof. Let us start by considering the case $\gamma=1$ in dimensions $d \geqslant 3$. Henceforth, $K_{1}$, $\mathcal{K}_{1}$ will be denoted by $K, \mathcal{K}$, respectively. Implicit constants may depend on $d, R$, as well as on the constant $C$ from (5.1). Consider the function $\delta(x)$ as in the proof of Lemma 3.1. Introduce a radial partition of unity on $B_{R},\left\{\phi_{j}\right\}_{j \geqslant 0}$, where $\phi_{j}=\delta\left(2^{j} R^{-1}\right.$ ) is supported where $2^{-j-1} R \leqslant|x| \leqslant 2^{-j+1} R$, and $\sum_{j \geqslant 0} \phi_{j}(x)=1$ for every $x \in B_{R} \backslash\{0\}$. Let $K_{j}=K \phi_{j}$, so that $\left\|K_{j}\right\|_{L^{\infty}} \leqslant 2^{j+1} R^{-1}\|H\|_{L^{\infty}}$, and $K_{j}$ is supported in the spherical shell

$$
A_{j}(R):=\left\{x \in \mathbb{R}^{d}: 2^{-j-1} R \leqslant|x| \leqslant 2^{-j+1} R\right\} .
$$

For $x, x^{\prime} \in A_{j}(R)$, we have that

$$
\begin{aligned}
\left|K_{j}(x)-K_{j}\left(x^{\prime}\right)\right|= & \left.|| x\right|^{-1} H(x) \phi_{j}(x)-\left|x^{\prime}\right|^{-1} H\left(x^{\prime}\right) \phi_{j}\left(x^{\prime}\right) \mid \\
\leqslant & \left.|| x\right|^{-1}-\left.\left|x^{\prime}\right|^{-1}|| H(x)\left|\phi_{j}(x)+\right| x^{\prime}\right|^{-1}\left|H(x)-H\left(x^{\prime}\right)\right| \phi_{j}(x) \\
& +\left|x^{\prime}\right|^{-1}\left|H\left(x^{\prime}\right)\right|\left|\phi_{j}(x)-\phi_{j}\left(x^{\prime}\right)\right| \\
& \lesssim\left|\frac{1}{|x|}-\frac{1}{\left|x^{\prime}\right|}\right|+2^{j}\left|x-x^{\prime}\right|^{\alpha}+2^{j}\left|\frac{x}{|x|}-\frac{x^{\prime}}{\left|x^{\prime}\right|}\right|+2^{2 j}\left|x-x^{\prime}\right| \\
& \lesssim 2^{2 j}\left|x-x^{\prime}\right|+2^{j}\left|x-x^{\prime}\right|^{\alpha}+2^{j}\left(\frac{1}{|x|}+\frac{1}{\left|x^{\prime}\right|}\right)\left|x-x^{\prime}\right| \\
& \lesssim 2^{2 j}\left|x-x^{\prime}\right|^{\alpha} .
\end{aligned}
$$

If $x, x^{\prime} \in B_{R}, x \in \operatorname{supp}\left(K_{j}\right)$ but $x^{\prime} \notin \operatorname{supp}\left(K_{j}\right)$, then $\left|K_{j}(x)-K_{j}\left(x^{\prime}\right)\right|=\left|K_{j}(x)\right| \lesssim 2^{j}$.

To each $K_{j}$ there is a corresponding operator $\mathcal{K}_{j}$, so that $\mathcal{K}=\sum_{j \geqslant 0} \mathcal{K}_{j}$. The claimed boundedness of $\mathcal{K}$ is ensured if the operator norms of the $\mathcal{K}_{j}$ are summable in $j$. In turn, the operator $\mathcal{K}_{j}$ is bounded on $L^{2}\left(\mathbb{S}^{d-1}\right)$, with operator norm $\left\|\mathcal{K}_{j}\right\|_{L^{2} \rightarrow L^{2}}=O\left(2^{-(d-2) j}\right)$. Indeed, by Schur's test, we have that

$$
\begin{aligned}
\sup _{v \in \mathbb{S}^{d-1}} \int_{\mathbb{S}^{d-1}}\left|K_{j}(\omega-v)\right| \mathrm{d} \sigma_{d-1}(\omega) & =\sup _{\omega \in \mathbb{S}^{d-1}} \int_{\mathbb{S}^{d-1}}\left|K_{j}(\omega-v)\right| \mathrm{d} \sigma_{d-1}(v) \\
& \lesssim 2^{j} \sup _{\omega \in \mathbb{S}^{d-1}} \int_{\mathbb{S}^{d-1}} \mathbb{1}_{\left\{2^{-j-1} R \leqslant|\omega-v| \leqslant 2^{-j+1} R\right\}}(v) \mathrm{d} \sigma_{d-1}(v)
\end{aligned}
$$




$$
\lesssim 2^{-(d-2) j}
$$

Moreover, $\mathcal{K}_{j}$ maps $L^{2}\left(\mathbb{S}^{d-1}\right)$ to $\Lambda_{\alpha}\left(\mathbb{S}^{d-1}\right)$. To see why this is the case, given $\omega, \omega^{\prime} \in \mathbb{S}^{d-1}$, define the sets

$$
\begin{aligned}
U\left(\omega, \omega^{\prime}\right) & :=\left\{v \in \mathbb{S}^{d-1}: \omega-v \in \operatorname{supp}\left(K_{j}\right), \omega^{\prime}-v \notin \operatorname{supp}\left(K_{j}\right)\right\}, \\
U\left(\omega^{\prime}, \omega\right) & :=\left\{v \in \mathbb{S}^{d-1}: \omega^{\prime}-v \in \operatorname{supp}\left(K_{j}\right), \omega-v \notin \operatorname{supp}\left(K_{j}\right)\right\}, \\
V & :=\left\{v \in \mathbb{S}^{d-1}: \omega-v, \omega^{\prime}-v \in \operatorname{supp}\left(K_{j}\right)\right\} .
\end{aligned}
$$

Observe that

$$
\sigma_{d-1}(V) \leqslant \int_{\mathbb{S} d-1} \mathbb{1}_{\left\{|\omega-v| \leqslant 2^{-j+1} R\right\}}(v) \mathrm{d} \sigma_{d-1}(v) \lesssim 2^{-(d-1) j} .
$$

On the other hand, and similar to (4.5), the following inclusion holds:

$$
\begin{aligned}
U\left(\omega, \omega^{\prime}\right) \subseteq\left\{v \in \mathbb{S}^{d-1}: 2^{-j-1} R-\left|\omega-\omega^{\prime}\right|\right. & \left.\leqslant\left|\omega^{\prime}-v\right| \leqslant 2^{-j-1} R\right\} \\
& \cup\left\{v \in \mathbb{S}^{d-1}: 2^{-j+1} R-\left|\omega-\omega^{\prime}\right| \leqslant|\omega-v| \leqslant 2^{-j+1} R\right\} .
\end{aligned}
$$

In particular, $\sigma_{d-1}\left(U\left(\omega, \omega^{\prime}\right)\right) \lesssim 2^{-(d-2) j}\left|\omega-\omega^{\prime}\right|$. By the same argument, we also have that $\sigma_{d-1}\left(U\left(\omega^{\prime}, \omega\right)\right) \lesssim 2^{-(d-2) j}\left|\omega-\omega^{\prime}\right|$. Then we may use (5.3) and estimate

$$
\begin{aligned}
\left|\left(\mathcal{K}_{j} f\right)(\omega)-\left(\mathcal{K}_{j} f\right)\left(\omega^{\prime}\right)\right| & \leqslant \int_{U\left(\omega, \omega^{\prime}\right) \cup U\left(\omega^{\prime}, \omega\right) \cup V}\left|K_{j}(\omega-v)-K_{j}\left(\omega^{\prime}-v\right)\right||f(v)| \mathrm{d} \sigma_{d-1}(v) \\
& \lesssim 2^{2 j}\left|\omega-\omega^{\prime}\right|^{\alpha} \int_{V}|f(v)| \mathrm{d} \sigma_{d-1}(v)+2^{j} \int_{U\left(\omega, \omega^{\prime}\right)}|f(v)| \mathrm{d} \sigma_{d-1}(v) \\
& \lesssim 2^{2 j}\left|\omega-\omega^{\prime}\right|^{\alpha} 2^{-\frac{d-1}{2} j}\|f\|_{L^{2}}+2^{-\frac{d-4}{2} j}\left|\omega-\omega^{\prime}\right|^{1 / 2}\|f\|_{L^{2}} \\
& \lesssim 2^{-\frac{d-5}{2} j}\left|\omega-\omega^{\prime}\right|^{\min \left\{\frac{1}{2}, \alpha\right\}}\|f\|_{L^{2}}
\end{aligned}
$$

No generality is lost in assuming that $\alpha \leqslant \frac{1}{2}$. Inequality (5.4) implies that $\mathcal{K}_{j}$ maps $L^{2}$ to $\mathcal{H}^{\alpha}$ boundedly and, moreover,

$$
\left\|\mathcal{K}_{j} f\right\|_{\mathcal{H}^{\alpha}} \lesssim\left\|\mathcal{K}_{j} f\right\|_{L^{2}}+2^{-\frac{d-5}{2} j}\|f\|_{L^{2}} \lesssim 2^{-\frac{d-5}{2} j}\|f\|_{L^{2}} .
$$

From the definition of the $\mathcal{H}^{s}$-spaces, one directly checks the following interpolation bounds:

$$
\|f\|_{\mathcal{H}^{\theta s+(1-\theta) t}} \leqslant C\|f\|_{\mathcal{H}^{s}}^{\theta}\|f\|_{\mathcal{H}^{t}}^{1-\theta} \text {, for all } \theta \in[0,1], 0 \leqslant s, t<1 .
$$

Using this to interpolate (5.5) with the $\mathcal{H}^{0}$-bound $\left\|\mathcal{K}_{j} f\right\|_{L^{2}} \lesssim 2^{-(d-2) j}\|f\|_{L^{2}}$ reveals that if $\delta>0$ is chosen sufficiently small depending on $d \in\{3,4,5\}$ and $\delta=\alpha$ if $d \geqslant 6$, then $\mathcal{K}_{j}$ maps $L^{2}$ to $\mathcal{H}^{\delta}$ boundedly, with operator norm $O\left(2^{-c j}\right)$ for some $c>0$ that does not depend on $j$. This implies that $\|\mathcal{K}\|_{L^{2} \rightarrow \mathcal{H}^{\delta}}<\infty$.

We now discuss the case $\gamma \in[0,1)$. If $d=2$, then the argument above works for the kernel $K_{\gamma}=|\cdot|^{-\gamma} H$ for any $\gamma \in(0,1)$, because the $L^{2} \rightarrow L^{2}$ operator norm of the corresponding $\mathcal{K}_{\gamma, j}$ is then $O\left(2^{-(1-\gamma) j}\right)$. If $d \geqslant 3$, then we write $K_{\gamma}=|\cdot|^{1-\gamma} K_{1}$ and see that the Hölder estimate for $K_{1}$ easily yields a corresponding statement for $K_{\gamma}$ for every $\gamma \in(0,1)$; in particular, the above argument also transfers. The argument for $\mathcal{K}_{0}$ is similar but simpler (details omitted). The proof of the lemma is now complete.

We are finally ready to establish a suitable replacement of Lemma 3.4 that handles the cases when $(d, m) \in \partial \mathfrak{U}$. 
Lemma 5.2. Given $(d, m) \in \partial \mathfrak{U}$, there exists $\alpha>0$ with the following property. If $\left\{h_{j}\right\}_{j=1}^{m} \subset \operatorname{Lip}\left(\mathbb{S}^{d-1}\right)$ and $g \in L^{2}\left(\mathbb{S}^{d-1}\right)$, then $\mathrm{M}\left(h_{1}, \ldots, h_{m}, g\right) \in \mathcal{H}^{\alpha}$. Moreover, the following estimate holds:

$$
\left\|\mathrm{M}\left(h_{1}, \ldots, h_{m}, g\right)\right\|_{\mathcal{H}^{\alpha}} \lesssim \prod_{j=1}^{m}\left\|h_{j}\right\|_{\operatorname{Lip}\left(\mathbb{S}^{d-1}\right)}\|g\|_{L^{2}\left(\mathbb{S}^{d-1}\right)} .
$$

Proof. We consider three distinct cases:

Case $d \geqslant 3, m=2$. From Corollary 4.2, the function $G=|\cdot|\left(h_{1} \sigma_{d-1} * h_{2} \sigma_{d-1}\right)$ satisfies

$$
\left|G(x)-G\left(x^{\prime}\right)\right| \lesssim\left\|h_{1}\right\|_{\text {Lip }}\left\|h_{2}\right\|_{\text {Lip }}\left(\left|x-x^{\prime}\right|^{1 / 2}+\left|\frac{x}{|x|}-\frac{x^{\prime}}{\left|x^{\prime}\right|}\right|\right) .
$$

The conclusion then follows from Lemma 5.1 with $\gamma=1$.

Case $(d, m)=(3,3)$. In view of Proposition 4.3, the function $h_{1} \sigma_{2} * h_{2} \sigma_{2} * h_{3} \sigma_{2}$ belongs to $\Lambda_{1 / 3}\left(\mathbb{R}^{3}\right)$. The conclusion then follows from Lemma 5.1 with $\gamma=0$.

Case $(d, m)=(2,4)$. In view of Proposition 4.6, given $\gamma>0$, there exists $\tau \in(0,1)$ such that the function $|\cdot|^{\gamma}\left(h_{1} \sigma_{1} * h_{2} \sigma_{1} * h_{3} \sigma_{1} * h_{4} \sigma_{1}\right)$ belongs to $\Lambda_{\tau}\left(\mathbb{R}^{2}\right)$. The conclusion then follows from Lemma 5.1 applied to any $\gamma \in(0,1)$.

\section{Smoothness of critical points}

This section is devoted to the proof of Theorem 1.1. Before starting the proof in earnest, we present two further results that will simplify the forthcoming analysis.

Given $(d, m) \in \mathfrak{U}$ and smooth functions $\left\{\varphi_{j}\right\}_{j=1}^{m} \subset C^{\infty}\left(\mathbb{S}^{d-1}\right)$, we define the linear operator $\mathrm{L}=\mathrm{L}\left[\varphi_{1}, \ldots, \varphi_{m}\right]: L^{2}\left(\mathbb{S}^{d-1}\right) \rightarrow L^{2}\left(\mathbb{S}^{d-1}\right)$ via

$$
\mathrm{L}\left[\varphi_{1}, \ldots, \varphi_{m}\right](g)=\mathrm{M}\left(\varphi_{1}, \ldots, \varphi_{m}, g\right) .
$$

Lemmas 3.4 and 5.2 together imply the bound $\|\mathrm{L}(g)\|_{\mathcal{H}^{\alpha}} \leqslant C\|g\|_{L^{2}}$, for some constant $C$ that depends on $d, m$, and on the functions $\left\{\varphi_{j}\right\}$. For our purposes, the precise dependence of the constant $C$ on $\left\{\varphi_{j}\right\}$ is not important; however, it is essential that $\mathrm{L}$ defines a bounded operator from $L^{2}\left(\mathbb{S}^{d-1}\right)$ to $\mathcal{H}^{\alpha}$ for some exponent $\alpha>0$ that is independent of the functions $\left\{\varphi_{j}\right\}$. Lemmas 3.4 and 5.2 can be recast in terms of the operator $\mathrm{L}$ as follows.

Corollary 6.1. Let $(d, m) \in \mathfrak{U}$. There exists $\alpha>0$ such that $\mathrm{L}\left[\varphi_{1}, \ldots, \varphi_{m}\right](g) \in \mathcal{H}^{\alpha}$ for any $\left\{\varphi_{j}\right\}_{j=1}^{m} \subset$ $C^{\infty}\left(\mathbb{S}^{d-1}\right)$ and $g \in L^{2}\left(\mathbb{S}^{d-1}\right)$. Moreover, the following estimate holds:

$$
\left\|\mathrm{L}\left[\varphi_{1}, \ldots, \varphi_{m}\right](g)\right\|_{\mathcal{H}^{\alpha}} \leqslant C\|g\|_{L^{2}(\mathbb{S} d-1)},
$$

where $C<\infty$ depends only on $d, m$, and on the functions $\left\{\varphi_{j}\right\}_{j=1}^{m}$.

We shall find it necessary to expand the expressions $(\Theta-I) \mathrm{M}\left(f_{1}, \ldots, f_{m+1}\right)$ and $(\Theta-$ $I)^{2} \mathrm{M}\left(f_{1}, \ldots, f_{m+1}\right)$, after a suitable decomposition $f_{j}=\varphi_{j, 0}+\varphi_{j, 1}, 1 \leqslant j \leqslant m+1$, has been performed. A model case for this situation is summarised in the following result. The list of $\left\{\varphi_{j}\right\}$ with the $i$ th term removed will be denoted by $\left[\varphi_{1}, \ldots, \stackrel{\circ}{i}_{i}, \ldots, \varphi_{m+1}\right]:=\left[\varphi_{1}, \ldots, \varphi_{i-1}, \varphi_{i+1}, \ldots, \varphi_{m+1}\right]$.

Lemma 6.2. Let $(d, m) \in \mathfrak{U}$, let $\varepsilon \in(0,1)$ and let $\left\{f_{j}\right\}_{j=1}^{m+1} \subset L^{2}\left(\mathbb{S}^{d-1}\right)$. For each $j$, decompose $f_{j}=\varphi_{j, 0}+\varphi_{j, 1}$, with $\left\|\varphi_{j, 0}\right\|_{L^{2}\left(\mathbb{S}^{d-1}\right)}<\varepsilon\left\|f_{j}\right\|_{L^{2}\left(\mathbb{S}^{d-1}\right)}$ and $\varphi_{j, 1} \in C^{\infty}\left(\mathbb{S}^{d-1}\right)$. Then, for any $\Theta \in S O(d)$, 
the following estimates hold:

$$
\begin{aligned}
\|(\Theta-I) \mathrm{M}\left(f_{1}, \ldots, f_{m+1}\right) & \|_{L^{2}\left(\mathbb{S}^{d-1}\right)} \\
& \lesssim \sum_{i=1}^{m+1}\left\|(\Theta-I) \mathrm{L}\left[\varphi_{1,1}, \ldots, \stackrel{\circ}{i}_{i, 1}, \ldots, \varphi_{m+1,1}\right]\left(\varphi_{i, 0}\right)\right\|_{L^{2}\left(\mathbb{S}^{d-1}\right)} \\
& +\sum_{i=1}^{m+1} \varepsilon\left\|(\Theta-I) \varphi_{i, 0}\right\|_{L^{2}\left(\mathbb{S}^{d-1}\right)} \prod_{j=1, j \neq i}^{m+1}\left\|f_{j}\right\|_{L^{2}\left(\mathbb{S}^{d-1}\right)} \\
& +\sum_{i=1}^{m+1}\left\|(\Theta-I) \varphi_{i, 1}\right\|_{L^{2}\left(\mathbb{S}^{d-1}\right)} \prod_{j=1, j \neq i}^{m+1}\left\|f_{j}\right\|_{L^{2}\left(\mathbb{S}^{d-1}\right)},
\end{aligned}
$$

and

$$
\begin{aligned}
& \left\|(\Theta-I)^{2} \mathrm{M}\left(f_{1}, \ldots, f_{m+1}\right)\right\|_{L^{2}\left(\mathbb{S}^{d-1}\right)} \\
& \lesssim \sum_{i=1}^{m+1}\left\|(\Theta-I) \mathrm{L}\left[\varphi_{1,1}, \ldots, \stackrel{\circ}{\varphi}_{i, 1}, \ldots, \varphi_{m+1,1}\right]\left((\Theta-I) \varphi_{i, 0}\right)\right\|_{L^{2}\left(\mathbb{S}^{d-1}\right)} \\
& \quad+\sum_{i=1}^{m+1} \varepsilon\left\|(\Theta-I)^{2} \varphi_{i, 0}\right\|_{L^{2}\left(\mathbb{S}^{d-1}\right)} \prod_{j=1, j \neq i}^{m+1}\left\|f_{j}\right\|_{L^{2}\left(\mathbb{S}^{d-1}\right)} \\
& \quad+\sum_{i=1}^{m+1}\left\|(\Theta-I)^{2} \varphi_{i, 1}\right\|_{L^{2}\left(\mathbb{S}^{d-1}\right)} \prod_{j=1, j \neq i}^{m+1}\left\|f_{j}\right\|_{L^{2}\left(\mathbb{S}^{d-1}\right)} \\
& \quad+\sum_{\substack{1 \leqslant i<j \leqslant m+1 \\
\left(\varepsilon_{i}, \varepsilon_{j}\right) \in\{0,1\}^{2}}}\left\|(\Theta-I) \varphi_{i, \varepsilon_{i}}\right\|_{L^{2}\left(\mathbb{S}^{d-1}\right)}\left\|(\Theta-I) \varphi_{j, \varepsilon_{j}}\right\|_{L^{2}\left(\mathbb{S}^{d-1}\right)} \prod_{k=1, k \notin\{i, j\}}^{m+1}\left\|f_{k}\right\|_{L^{2}\left(\mathbb{S}^{d-1}\right)}
\end{aligned}
$$

Estimates (6.2) and (6.3) exhibit a certain degree of asymmetry with respect to the role played by the functions $\varphi_{i, 0}$ and $\varphi_{i, 1}$. This is in order to ensure that the less smooth terms $\left\|(\Theta-I) \varphi_{i, 0}\right\|_{L^{2}\left(\mathbb{S}^{d-1}\right)}$ and $\left\|(\Theta-I)^{2} \varphi_{i, 0}\right\|_{L^{2}\left(\mathbb{S}^{d-1}\right)}$ always carry a mitigating factor of $\varepsilon$.

Proof of Lemma 6.2. Decompose each $f_{j}=\varphi_{j, 0}+\varphi_{j, 1}$ as in the statement of the lemma. Substituting this into $g:=\mathbf{M}\left(f_{1}, \ldots, f_{m+1}\right)$ and using the multilinearity of $\mathbf{M}$ together with the permutation symmetry (3.3), we have that

$$
\begin{aligned}
g= & \sum_{\left(\varepsilon_{1}, \ldots, \varepsilon_{m+1}\right) \in\{0,1\}^{m+1}} \mathrm{M}\left(\varphi_{1, \varepsilon_{1}}, \ldots, \varphi_{m+1, \varepsilon_{m+1}}\right) \\
= & \mathrm{M}\left(\varphi_{1,1}, \ldots, \varphi_{m+1,1}\right)+\sum_{i=1}^{m+1} \mathrm{~L}\left[\varphi_{1,1}, \ldots, \stackrel{\circ}{\varphi}_{i, 1}, \ldots, \varphi_{m+1,1}\right]\left(\varphi_{i, 0}\right) \\
& +\sum_{\substack{\left(\varepsilon_{1}, \ldots, \varepsilon_{m+1}\right) \in\{0,1\}^{m+1} \\
\varepsilon_{1}+\cdots+\varepsilon_{m+1} \leqslant m-1}} \mathrm{M}\left(\varphi_{1, \varepsilon_{1}}, \ldots, \varphi_{m+1, \varepsilon_{m+1}}\right) .
\end{aligned}
$$

The first, second and third summands in the latter expression correspond to those cases in which exactly none, one or at least two of the $\varepsilon_{i}$ s are equal to 0 , respectively. Therefore,

$$
(\Theta-I) g=(\Theta-I) \mathrm{M}\left(\varphi_{1,1}, \ldots, \varphi_{m+1,1}\right)+\sum_{i=1}^{m+1}(\Theta-I) \mathrm{L}\left[\varphi_{1,1}, \ldots, \dot{\varphi}_{i, 1}, \ldots, \varphi_{m+1,1}\right]\left(\varphi_{i, 0}\right)
$$




$$
+\sum_{\substack{\left(\varepsilon_{1}, \ldots, \varepsilon_{m+1}\right) \in\{0,1\}^{m+1} \\ \varepsilon_{1}+\cdots+\varepsilon_{m+1} \leqslant m-1}}(\Theta-I) \mathrm{M}\left(\varphi_{1, \varepsilon_{1}}, \ldots, \varphi_{m+1, \varepsilon_{m+1}}\right)
$$

In order to $L^{2}$-bound the terms coming from the latter sum in (6.4), we appeal to identity (3.5) for each summand and obtain a further sum of terms of the form

$$
\mathrm{M}\left(\varphi_{1, \varepsilon_{1}}, \ldots, \varphi_{i-1, \varepsilon_{i-1}},(\Theta-I) \varphi_{i, \varepsilon_{i}}, \Theta \varphi_{i+1, \varepsilon_{i+1}}, \ldots, \Theta \varphi_{m+1, \varepsilon_{m+1}}\right)
$$

The corresponding $L^{2}$-norms can be bounded via the basic estimate (3.9), yielding

$$
\begin{array}{r}
\left\|\mathrm{M}\left(\varphi_{1, \varepsilon_{1}}, \ldots, \varphi_{i-1, \varepsilon_{i-1}},(\Theta-I) \varphi_{i, \varepsilon_{i}}, \Theta \varphi_{i+1, \varepsilon_{i+1}}, \ldots, \Theta \varphi_{m+1, \varepsilon_{m+1}}\right)\right\|_{L^{2}\left(\mathbb{S}^{d-1}\right)} \\
\lesssim\left\|(\Theta-I) \varphi_{i, \varepsilon_{i}}\right\|_{L^{2}\left(\mathbb{S}^{d-1}\right)} \prod_{j=1, j \neq i}^{m+1}\left\|\varphi_{j, \varepsilon_{j}}\right\|_{L^{2}\left(\mathbb{S}^{d-1}\right)} .
\end{array}
$$

As noted before, the condition $\varepsilon_{1}+\cdots+\varepsilon_{m+1} \leqslant m-1$ implies the existence of at least two distinct indices $i^{\prime} \neq j^{\prime}$ such that $\varepsilon_{i^{\prime}}=\varepsilon_{j^{\prime}}=0$. In this way, (6.5) is bounded by

$$
\varepsilon\left\|(\Theta-I) \varphi_{i, 0}\right\|_{L^{2}\left(\mathbb{S}^{d-1}\right)} \prod_{j=1, j \neq i}^{m+1}\left\|f_{j}\right\|_{L^{2}\left(\mathbb{S}^{d-1}\right)}
$$

if $\varepsilon_{i}=0$ or even better by

$$
\varepsilon^{2}\left\|(\Theta-I) \varphi_{i, 1}\right\|_{L^{2}\left(\mathbb{S}^{d-1}\right)} \prod_{j=1, j \neq i}^{m+1}\left\|f_{j}\right\|_{L^{2}\left(\mathbb{S}^{d-1}\right)}
$$

if $\varepsilon_{i}=1$. Finally, observe that

$$
\left\|(\Theta-I) \mathrm{M}\left(\varphi_{1,1}, \ldots, \varphi_{m+1,1}\right)\right\|_{L^{2}\left(\mathbb{S}^{d-1}\right)} \leqslant \sum_{i=1}^{m+1}\left\|(\Theta-I) \varphi_{i, 1}\right\|_{L^{2}(\mathbb{S} d-1)} \prod_{j=1, j \neq i}^{m+1}\left\|f_{j}\right\|_{L^{2}(\mathbb{S} d-1)} .
$$

Adding up all of the contributions, we obtain (6.2). Considering now (6.3), we start from (6.4), apply $\Theta-I$ to both sides and obtain

$$
\begin{aligned}
(\Theta-I)^{2} g=(\Theta & -I)^{2} \mathrm{M}\left(\varphi_{1,1}, \ldots, \varphi_{m+1,1}\right) \\
& +\sum_{i=1}^{m+1}(\Theta-I)^{2} \mathrm{M}\left(\varphi_{1,1}, \ldots, \stackrel{\circ}{i, 1}_{i}, \ldots, \varphi_{m+1,1}, \varphi_{i, 0}\right) \\
& +\sum_{\substack{\left(\varepsilon_{1}, \ldots, \varepsilon_{m+1}\right) \in\{0,1\}^{m+1} \\
\varepsilon_{1}+\cdots+\varepsilon_{m+1} \leqslant m-1}}(\Theta-I)^{2} \mathrm{M}\left(\varphi_{1, \varepsilon_{1}}, \ldots, \varphi_{m+1, \varepsilon_{m+1}}\right) .
\end{aligned}
$$

Using (3.5) twice together with the basic estimate (3.9), the first term on the latter right-hand side can be bounded as follows:

$$
\begin{aligned}
& \left\|(\Theta-I)^{2} \mathrm{M}\left(\varphi_{1,1}, \ldots, \varphi_{m+1,1}\right)\right\|_{L^{2}\left(\mathbb{S}^{d-1}\right)} \\
& \lesssim \sum_{\substack{1 \leqslant i<j \leqslant m+1 \\
\text { 2021.7 Published online by Cambridge University Press }}}\left\|(\Theta-I) \varphi_{i, 1}\right\|_{L^{2}\left(\mathbb{S}^{d-1}\right)}\left\|(\Theta-I) \varphi_{j, 1}\right\|_{L^{2}\left(\mathbb{S}^{d-1}\right)} \prod_{k: k \notin\{i, j\}}\left\|f_{k}\right\|_{L^{2}\left(\mathbb{S}^{d-1}\right)}
\end{aligned}
$$




$$
+\sum_{i=1}^{m+1}\left\|(\Theta-I)^{2} \varphi_{i, 1}\right\|_{L^{2}\left(\mathbb{S}^{d-1}\right)} \prod_{j: j \neq i}\left\|f_{j}\right\|_{L^{2}\left(\mathbb{S}^{d-1}\right)} .
$$

An upper bound similar to the preceding one also applies to each term from the third sum in (6.6), but this can be refined as follows:

$$
\begin{aligned}
& \left\|(\Theta-I)^{2} \mathrm{M}\left(\varphi_{1, \varepsilon_{1}}, \ldots, \varphi_{m+1, \varepsilon_{m+1}}\right)\right\|_{L^{2}\left(\mathbb{S}^{d-1}\right)} \\
& \lesssim \sum_{1 \leqslant i<j \leqslant m+1}\left\|(\Theta-I) \varphi_{i, \varepsilon_{i}}\right\|_{L^{2}\left(\mathbb{S}^{d-1}\right)}\left\|(\Theta-I) \varphi_{j, \varepsilon_{j}}\right\|_{L^{2}\left(\mathbb{S}^{d-1}\right)} \prod_{k: k \notin\{i, j\}}\left\|f_{k}\right\|_{L^{2}\left(\mathbb{S}^{d-1}\right)} \\
& \quad+\sum_{i=1, \varepsilon_{i}=1}^{m+1} \varepsilon^{2}\left\|(\Theta-I)^{2} \varphi_{i, 1}\right\|_{L^{2}\left(\mathbb{S}^{d-1}\right)} \prod_{j: j \neq i}\left\|f_{j}\right\|_{L^{2}\left(\mathbb{S}^{d-1}\right)} \\
& \quad+\sum_{i=1, \varepsilon_{i}=0}^{m+1} \varepsilon\left\|(\Theta-I)^{2} \varphi_{i, 0}\right\|_{L^{2}\left(\mathbb{S}^{d-1}\right)} \prod_{j: j \neq i}\left\|f_{j}\right\|_{L^{2}\left(\mathbb{S}^{d-1}\right)} .
\end{aligned}
$$

Lastly, each of the terms coming from the second sum in (6.6) can be bounded as follows:

$$
\begin{aligned}
& \left\|(\Theta-I)^{2} \mathrm{M}\left(\varphi_{1,1}, \ldots, \stackrel{\circ}{i, 1}, \ldots, \varphi_{m+1,1}, \varphi_{i, 0}\right)\right\|_{L^{2}\left(\mathbb{S}^{d-1}\right)} \\
& \lesssim \sum_{\substack{1 \leqslant j<k \leqslant m+1 \\
j \neq i, k \neq i}} \varepsilon\left\|(\Theta-I) \varphi_{j, 1}\right\|_{L^{2}\left(\mathbb{S}^{d-1}\right)}\left\|(\Theta-I) \varphi_{k, 1}\right\|_{L^{2}\left(\mathbb{S}^{d-1}\right)} \prod_{\ell \notin\{j, k\}}\left\|f_{\ell}\right\|_{L^{2}\left(\mathbb{S}^{d-1}\right)} \\
& \quad+\sum_{j=1, j \neq i}^{m+1}\left\|(\Theta-I) \varphi_{i, 0}\right\|_{L^{2}\left(\mathbb{S}^{d-1}\right)}\left\|(\Theta-I) \varphi_{j, 1}\right\|_{L^{2}\left(\mathbb{S}^{d-1}\right)} \prod_{k \notin\{i, j\}}\left\|f_{k}\right\|_{L^{2}\left(\mathbb{S}^{d-1}\right)} \\
& \quad+\left\|(\Theta-I) \operatorname{L}\left[\varphi_{1,1}, \ldots, \stackrel{\circ}{i, 1}_{i}, \ldots, \varphi_{m+1,1}\right]\left((\Theta-I) \varphi_{i, 0}\right)\right\|_{L^{2}\left(\mathbb{S}^{d-1}\right)} .
\end{aligned}
$$

Adding up all of the contributions yields (6.3). This completes the proof of the lemma.

\subsection{Proof of Theorem 1.1}

We are now ready to start with the proof of Theorem 1.1 in earnest. As a first step, we establish an initial regularity kick. Henceforth we assume the parameter $\lambda$ in equation (1.12) to be nonzero, in which case $\lambda$ can be absorbed into the function $a$; see the final remark in Subsection 6.3. We are thus interested in solutions of the equation

$$
a \cdot \mathrm{M}\left(R^{k_{1}}(f), \ldots, R^{k_{m+1}}(f)\right)=f, \quad \sigma_{d-1} \text {-a.e. on } \mathbb{S}^{d-1} .
$$

Proposition 6.3. Let $(d, m) \in \mathfrak{U}$ and $\left(k_{1}, \ldots, k_{m+1}\right) \in\{0,1\}^{m+1}$. Assume that a $\in \Lambda_{\kappa}\left(\mathbb{S}^{d-1}\right)$ for some $\kappa \in(0,1)$. Then, given any complex-valued solution $f \in L^{2}\left(\mathbb{S}^{d-1}\right)$ of equation (6.7), there exists $s>0$ such that $f \in \mathcal{H}^{s}$.

Proof. Let $f \in L^{2}\left(\mathbb{S}^{d-1}\right)$ be a complex-valued solution of $(6.7)$, and let $\varepsilon \in(0,1)$ be a small constant, to be chosen in the course of the argument. We may decompose $f=g_{\varepsilon}+\varphi_{\varepsilon}$, where $\left\|g_{\varepsilon}\right\|_{L^{2}}<\varepsilon\|f\|_{L^{2}}$, and $\varphi_{\varepsilon} \in C^{\infty}$. In this way, we have that $\left\|\varphi_{\varepsilon}\right\|_{L^{2}} \leqslant(1+\varepsilon)\|f\|_{L^{2}} \leqslant 2\|f\|_{L^{2}}$; it is important that the latter bound is independent of $\varepsilon$. By multilinearity of $\mathrm{M}$, no generality is lost in assuming that $f$ is $L^{2}$ normalised, $\|f\|_{L^{2}}=1$. In (6.7), we further suppose that $k_{i}=0$ for every $1 \leqslant i \leqslant m+1$. This assumption is made for notational purposes only, because the exact same argument applies in general. ${ }^{5}$ Substituting

${ }^{5}$ Note that the operator $R$ is a linear isometry and that $\|(\Theta-I) f\|_{L^{2}}=\left\|(\Theta-I) f_{\star}\right\|_{L^{2}}$ for every $\Theta \in \operatorname{SO}(d)$. 
$f=g_{\varepsilon}+\varphi_{\varepsilon}$ into the right-hand side of (6.7), we then see that the function $g_{\varepsilon}$ satisfies the equation

$$
g_{\varepsilon}=a \cdot \mathrm{M}(f, \ldots, f)-\varphi_{\varepsilon} .
$$

Given $\Theta \in \mathrm{SO}(d)$, apply $\Theta-I$ to both sides of the latter identity, yielding

$$
(\Theta-I) g_{\varepsilon}=(\Theta-I) a \cdot \Theta \mathrm{M}(f, \ldots, f)+a \cdot(\Theta-I) \mathrm{M}(f, \ldots, f)-(\Theta-I) \varphi_{\varepsilon} .
$$

Consequently,

$$
\begin{aligned}
\left\|(\Theta-I) g_{\varepsilon}\right\|_{L^{2}\left(\mathbb{S}^{d-1}\right)} \leqslant & \|(\Theta-I) a\|_{L^{\infty}\left(\mathbb{S}^{d-1}\right)}\|\mathrm{M}(f, \ldots, f)\|_{L^{2}\left(\mathbb{S}^{d-1}\right)}+\left\|(\Theta-I) \varphi_{\varepsilon}\right\|_{L^{2}\left(\mathbb{S}^{d-1}\right)} \\
& +\|a\|_{L^{\infty}(\mathbb{S} d-1)}\|(\Theta-I) \mathrm{M}(f, \ldots, f)\|_{L^{2}(\mathbb{S} d-1)} .
\end{aligned}
$$

We estimate the third summand on the right-hand side of the latter inequality with the help of Lemma 6.2 , yielding

$$
\begin{aligned}
\left\|(\Theta-I) g_{\varepsilon}\right\|_{L^{2}\left(\mathbb{S}^{d-1}\right)} \lesssim\|(\Theta-I) a\|_{L^{\infty}\left(\mathbb{S}^{d-1}\right)}+\left(1+\|a\|_{L^{\infty}\left(\mathbb{S}^{d-1}\right)}\right)\left\|(\Theta-I) \varphi_{\varepsilon}\right\|_{L^{2}\left(\mathbb{S}^{d-1}\right)} \\
+\|a\|_{L^{\infty}\left(\mathbb{S}^{d-1}\right)}\left(\left\|(\Theta-I) \mathrm{L}\left[\varphi_{\varepsilon}, \ldots, \varphi_{\varepsilon}\right]\left(g_{\varepsilon}\right)\right\|_{L^{2}\left(\mathbb{S}^{d-1}\right)}+\varepsilon\left\|(\Theta-I) g_{\varepsilon}\right\|_{L^{2}\left(\mathbb{S}^{d-1}\right)}\right) .
\end{aligned}
$$

We may now choose $\varepsilon \in(0,1)$ small enough, depending on $d, m$ and $\|a\|_{L^{\infty}}$, so that the last term on the right-hand side can be absorbed into the left-hand side, yielding

$$
\begin{aligned}
\left\|(\Theta-I) g_{\varepsilon}\right\|_{L^{2}\left(\mathbb{S}^{d-1}\right)} \lesssim & \|(\Theta-I) a\|_{L^{\infty}\left(\mathbb{S}^{d-1}\right)}+\left(1+\|a\|_{L^{\infty}\left(\mathbb{S}^{d-1}\right)}\right)\left\|(\Theta-I) \varphi_{\varepsilon}\right\|_{L^{2}\left(\mathbb{S}^{d-1}\right)} \\
& +\|a\|_{L^{\infty}\left(\mathbb{S}^{d-1}\right)}\left\|(\Theta-I) \mathrm{L}\left[\varphi_{\varepsilon}, \ldots, \varphi_{\varepsilon}\right]\left(g_{\varepsilon}\right)\right\|_{L^{2}\left(\mathbb{S}^{d-1}\right)} .
\end{aligned}
$$

Choose $s \in(0,1)$ in such a way that $s \leqslant \kappa$ and $\mathrm{L}\left[\varphi_{\varepsilon}, \ldots, \varphi_{\varepsilon}\right]$ is bounded from $L^{2}$ to $\mathcal{H}^{s}$, as promised by Corollary 6.1. Such an $s$ can be chosen independent of the function $\varphi_{\varepsilon}$ and therefore does not depend on $\varepsilon$ either (but the implicit constant may depend on $\varepsilon$, which we now take as fixed). Setting $\Theta=e^{t X_{i, j}}$ for some $1 \leqslant i<j \leqslant d$, multiplying by $|t|^{-s}$ and taking the supremum over $|t| \in[0,1]$ yields

$$
\begin{aligned}
& \sup _{|t| \leqslant 1}|t|^{-s}\left\|\left(e^{t X_{i, j}}-I\right) g_{\varepsilon}\right\|_{L^{2}\left(\mathbb{S}^{d-1}\right)} \\
& \quad \lesssim\|a\|_{\Lambda_{s}\left(\mathbb{S}^{d-1}\right)}+\left(1+\|a\|_{L^{\infty}\left(\mathbb{S}^{d-1}\right)}\right)\left\|\varphi_{\varepsilon}\right\|_{\mathcal{H}^{s}}+C_{\varepsilon}\|a\|_{L^{\infty}\left(\mathbb{S}^{d-1}\right)}\left\|g_{\varepsilon}\right\|_{L^{2}(\mathbb{S} d-1)}<\infty
\end{aligned}
$$

Here we are using that the $\Lambda_{s}$-norm can be controlled by the $\Lambda_{\kappa}$-norm because $s \leqslant \kappa$. Estimate (6.8) implies that $g_{\varepsilon} \in \mathcal{H}^{s}$ and therefore $f \in \mathcal{H}^{s}$ as well. The proof of the proposition is now complete.

Remark 6.4. If $(d, m) \in \mathfrak{U} \backslash \partial \mathfrak{U}$, then there is an automatic gain in the initial regularity of any complexvalued $f \in L^{2}\left(\mathbb{S}^{d-1}\right)$ solution of equation (6.7). Indeed, we claim that in that case $f$ necessarily coincides with a continuous function on $\mathbb{S}^{d-1}$. To see why this must be so, start by considering the case $d, m \geqslant 3$. Writing $m+1=(m-1)+2$, where $m-1 \geqslant 2$, we see that the convolution product on the left-hand side of (1.12) can be written as

$$
\left(R^{k_{1}}(f) \sigma_{d-1} * \cdots * R^{k_{m-1}}(f) \sigma_{d-1}\right) *\left(R^{k_{m}}(f) \sigma_{d-1} * R^{k_{m+1}}(f) \sigma_{d-1}\right) .
$$

Because each of the two functions in the preceding convolution belongs to $L^{2}\left(\mathbb{R}^{d}\right)$, their convolution defines a continuous function of bounded support on $\mathbb{R}^{d}$. It follows that its restriction to the unit sphere also defines a continuous function on $\mathbb{S}^{d-1}$, as claimed. An analogous argument works for the case $d=2$ and $m \geqslant 5$.

The second main step is a bootstrapping procedure that will complete the proof of Theorem 1.1. Indeed, in light of Remark 2.2, Propositions 6.3 and 6.5 together imply that a solution $f$ of equation (6.7) 
(and therefore of equation (1.12) if $\lambda \neq 0$ ) satisfies $f \in H^{r}$ for every $r \geqslant 0$. From Sobolev embedding - see, for example, [18, Theorem 2.7] - it then follows that $f \in C^{\infty}\left(\mathbb{S}^{d-1}\right)$.

Proposition 6.5. Let $(d, m) \in \mathfrak{H}$. Let $\left(k_{1}, \ldots, k_{m+1}\right) \in\{0,1\}^{m+1}, \lambda \in \mathbb{C} \backslash\{0\}$ and $a \in C^{\infty}\left(\mathbb{S}^{d-1}\right)$. Then there exists $\alpha>0$ with the following property. Let $f$ be a solution of equation (6.7) satisfying $f \in \mathcal{H}^{s}$ for some $s>0$. Then $f \in \mathcal{H}^{t}$ for every $t \in[0, s+\min \{s-\lfloor s\rfloor, \alpha\}] \backslash \mathbb{Z}$.

Proof. We make a few initial simplifications. Firstly, we consider the special case $a \equiv 1$ only, because the general case $a \in C^{\infty}\left(\mathbb{S}^{d-1}\right)$ brings no additional complications, as shown by the proof of Proposition 6.3. Secondly, we further assume that $k_{i}=0$, for every $1 \leqslant i \leqslant m+1$; this considerably simplifies the forthcoming notation but changes nothing fundamental in the analysis. Thirdly, we start by supposing that $s \in(0,1)$. The case $s \geqslant 1$ will be dealt with at a later stage in the proof.

Assume $\|f\|_{L^{2}}=1$, and let $\varepsilon \in(0,1)$, to be chosen in the course of the argument. Decompose $f=g_{\varepsilon}+\varphi_{\varepsilon}$, with $\varphi_{\varepsilon} \in C^{\infty}\left(\mathbb{S}^{d-1}\right)$ and $\left\|g_{\varepsilon}\right\|_{L^{2}}<\varepsilon$. In particular, $\left\|\varphi_{\varepsilon}\right\|_{L^{2}} \leqslant 2$. Because $f \in \mathcal{H}^{s}$, it follows that $g_{\varepsilon} \in \mathcal{H}^{S}$ as well. The equation satisfied by $g_{\varepsilon}$ is

$$
g_{\varepsilon}=\mathrm{M}(f, \ldots, f)-\varphi_{\varepsilon} .
$$

Given $\Theta \in \mathrm{SO}(d)$, we have that

$$
(\Theta-I)^{2} g_{\varepsilon}=(\Theta-I)^{2} \mathrm{M}(f, \ldots, f)-(\Theta-I)^{2} \varphi_{\varepsilon}
$$

and, therefore,

$$
\left\|(\Theta-I)^{2} g_{\mathcal{E}}\right\|_{L^{2}\left(\mathbb{S}^{d-1}\right)} \leqslant\left\|(\Theta-I)^{2} \mathrm{M}(f, \ldots, f)\right\|_{L^{2}\left(\mathbb{S}^{d-1}\right)}+\left\|(\Theta-I)^{2} \varphi_{\mathcal{E}}\right\|_{L^{2}\left(\mathbb{S}^{d-1}\right)} .
$$

Using Lemma 6.2 to estimate the first term on the right-hand side of the preceding inequality, we obtain

$$
\begin{aligned}
\left\|(\Theta-I)^{2} g_{\varepsilon}\right\|_{L^{2}\left(\mathbb{S}^{d-1}\right)} & \lesssim\left\|(\Theta-I) \varphi_{\varepsilon}\right\|_{L^{2}\left(\mathbb{S}^{d-1}\right)}^{2}+\left\|(\Theta-I)^{2} \varphi_{\varepsilon}\right\|_{L^{2}\left(\mathbb{S}^{d-1}\right)} \\
+ & \left\|(\Theta-I) \varphi_{\varepsilon}\right\|_{L^{2}\left(\mathbb{S}^{d-1}\right)}\left\|(\Theta-I) g_{\varepsilon}\right\|_{L^{2}\left(\mathbb{S}^{d-1}\right)}+\left\|(\Theta-I) g_{\varepsilon}\right\|_{L^{2}\left(\mathbb{S}^{d-1}\right)}^{2} \\
& +\left\|(\Theta-I) \mathrm{L}\left[\varphi_{\varepsilon}, \ldots, \varphi_{\varepsilon}\right]\left((\Theta-I) g_{\varepsilon}\right)\right\|_{L^{2}\left(\mathbb{S}^{d-1}\right)}+\varepsilon\left\|(\Theta-I)^{2} g_{\varepsilon}\right\|_{L^{2}\left(\mathbb{S}^{d-1}\right)}^{2} .
\end{aligned}
$$

Now choose $\varepsilon \in(0,1)$ small enough, depending on $d, m$, in such a way that the last term on the latter left-hand side can be absorbed into the right-hand side. With such a choice of $\varepsilon$, the following inequality holds:

$$
\begin{aligned}
\left\|(\Theta-I)^{2} g_{\varepsilon}\right\|_{L^{2}\left(\mathbb{S}^{d-1}\right)} \lesssim & \left\|(\Theta-I) \varphi_{\varepsilon}\right\|_{L^{2}\left(\mathbb{S}^{d-1}\right)}^{2}+\left\|(\Theta-I)^{2} \varphi_{\varepsilon}\right\|_{L^{2}\left(\mathbb{S}^{d-1}\right)} \\
& +\left\|(\Theta-I) \varphi_{\varepsilon}\right\|_{L^{2}\left(\mathbb{S}^{d-1}\right)}\left\|(\Theta-I) g_{\varepsilon}\right\|_{L^{2}\left(\mathbb{S}^{d-1}\right)}+\left\|(\Theta-I) g_{\varepsilon}\right\|_{L^{2}\left(\mathbb{S}^{d-1}\right)}^{2} \\
& +\left\|(\Theta-I) \mathrm{L}\left[\varphi_{\varepsilon}, \ldots, \varphi_{\varepsilon}\right]\left((\Theta-I) g_{\varepsilon}\right)\right\|_{L^{2}\left(\mathbb{S}^{d-1}\right)} .
\end{aligned}
$$

Now that $\varepsilon$ has been fixed, Corollary 6.1 implies that the operator $\mathrm{L}\left[\varphi_{\varepsilon}, \ldots, \varphi_{\varepsilon}\right]$ is bounded from $L^{2}$ to $\mathcal{H}^{\alpha}$, for some $\alpha \in(0,1)$ independent of $\varepsilon$.

Set $\delta=\min \{s, \alpha\}$, where $\alpha$ is as in the previous paragraph. In particular, $\mathrm{L}\left[\varphi_{\varepsilon}, \ldots, \varphi_{\varepsilon}\right]$ is bounded from $L^{2}$ to $\mathcal{H}^{\delta}$, with operator norm that may depend on $\varepsilon$. Henceforth we consider $\Theta=\Theta(t)=e^{t X_{k, \ell}}$, $1 \leqslant k<\ell \leqslant d$ and $|t| \leqslant 1$. The following estimate holds:

$$
\begin{aligned}
\|(\Theta-I) \mathrm{L}\left[\varphi_{\varepsilon}, \ldots, \varphi_{\varepsilon}\right] & \left((\Theta-I) g_{\varepsilon}\right) \|_{L^{2}\left(\mathbb{S}^{d-1}\right)} \\
& \leqslant|t|^{\delta} \sup _{|\tau| \leqslant 1}|\tau|^{-\delta}\left\|(\Theta(\tau)-I) \mathrm{L}\left[\varphi_{\varepsilon}, \ldots, \varphi_{\varepsilon}\right]\left((\Theta(t)-I) g_{\varepsilon}\right)\right\|_{L^{2}\left(\mathbb{S}^{d-1}\right)} \\
& \leqslant|t|^{\delta}\left\|\mathrm{L}\left[\varphi_{\varepsilon}, \ldots, \varphi_{\varepsilon}\right]\left((\Theta(t)-I) g_{\varepsilon}\right)\right\|_{\mathcal{H}^{\delta}} \\
& \leqslant C_{\varepsilon}|t|^{\delta}\left\|(\Theta-I) g_{\varepsilon}\right\|_{L^{2}\left(\mathbb{S}^{d-1}\right)}
\end{aligned}
$$




$$
\leqslant C_{\varepsilon}|t|^{\delta+s}\left\|g_{\varepsilon}\right\|_{\mathcal{H}^{s}}
$$

Multiplying (6.9) by $|t|^{-(s+\delta)}$ yields

$$
\begin{aligned}
& |t|^{-(s+\delta)}\left\|(\Theta-I)^{2} g_{\varepsilon}\right\|_{L^{2}\left(\mathbb{S}^{d-1}\right)} \\
& \quad \lesssim|t|^{-\delta}\left\|(\Theta-I) \varphi_{\varepsilon}\right\|_{L^{2}\left(\mathbb{S}^{d-1}\right)}|t|^{-s}\left\|(\Theta-I) \varphi_{\varepsilon}\right\|_{L^{2}\left(\mathbb{S}^{d-1}\right)}+|t|^{-(s+\delta)}\left\|(\Theta-I)^{2} \varphi_{\varepsilon}\right\|_{L^{2}\left(\mathbb{S}^{d-1}\right)} \\
& \quad+|t|^{-\delta}\left\|(\Theta-I) \varphi_{\varepsilon}\right\|_{L^{2}\left(\mathbb{S}^{d-1}\right)}|t|^{-s}\left\|(\Theta-I) g_{\varepsilon}\right\|_{L^{2}\left(\mathbb{S}^{d-1}\right)} \\
& \quad+|t|^{-\delta}\left\|(\Theta-I) g_{\varepsilon}\right\|_{L^{2}\left(\mathbb{S}^{d-1}\right)}|t|^{-s}\left\|(\Theta-I) g_{\varepsilon}\right\|_{L^{2}\left(\mathbb{S}^{d-1}\right)}+C_{\varepsilon}\left\|g_{\varepsilon}\right\|_{\mathcal{H}^{s}} .
\end{aligned}
$$

Now take the supremum over $|t| \leqslant 1$ and use the facts that $\varphi_{\varepsilon} \in \mathcal{H}^{r}$ for all $0 \leqslant r \notin \mathbb{Z}$ and $g_{\varepsilon} \in \mathcal{H}^{s} \cap \mathcal{H}^{\delta}$ (recall that $\delta \leqslant s$ ). Invoking the characterisation of the $\mathcal{H}^{s+\delta}$-norm by means of second differences as detailed in Subsection 6.2, which applies because $s+\delta \in(0,2)$, we obtain that

$$
\begin{aligned}
\sup _{|t| \leqslant 1}|t|^{-(s+\delta)}\left\|(\Theta-I)^{2} g_{\varepsilon}\right\|_{L^{2}\left(\mathbb{S}^{d-1}\right)} \lesssim & \left\|\varphi_{\varepsilon}\right\|_{\mathcal{H}^{\delta}}\left\|\varphi_{\varepsilon}\right\|_{\mathcal{H}^{s}}+\left\|\varphi_{\varepsilon}\right\|_{\mathcal{H}^{s+\delta}}+\left\|\varphi_{\varepsilon}\right\|_{\mathcal{H}^{\delta}}\left\|g_{\varepsilon}\right\|_{\mathcal{H}^{s}} \\
& +\left\|g_{\varepsilon}\right\|_{\mathcal{H}^{\delta}}\left\|g_{\varepsilon}\right\|_{\mathcal{H}^{s}}+C_{\varepsilon}\|g\|_{\mathcal{H}^{s}}<\infty .
\end{aligned}
$$

In this way, again via second differences, we see that $g_{\varepsilon} \in \mathcal{H}^{s+\delta}$ and therefore $f \in \mathcal{H}^{s+\delta}$ as well. ${ }^{6}$ This concludes the proof of the proposition in the special case when $s \in(0,1)$.

Repeated applications of the previous step reveal that if $f \in \mathcal{H}^{s}$ for some $s \in(0,1)$, then $f \in \mathcal{H}^{1+\gamma}$ for some $\gamma \in(0,1)$. We complete the proof of the proposition by induction. In order to treat exponents $s=k+\gamma$, with $k \in \mathbb{N}$ and $\gamma \in(0,1)$, we use the product rule (3.7) and differentiate $k$ times identity (6.7) with respect to $X \in\left\{X_{i, j}: 1 \leqslant i<j \leqslant d\right\}$, thus obtaining an equation for $X^{k} f \in \mathcal{H}^{\gamma}$. Decomposing $X^{k} f=g_{\varepsilon}+\varphi_{\varepsilon}$, with $\varphi_{\varepsilon} \in C^{\infty}\left(\mathbb{S}^{d-1}\right)$ and $\left\|g_{\varepsilon}\right\|_{L^{2}} \leqslant \varepsilon\left\|X^{k} f\right\|_{L^{2}}$, we can use the same method as before to show that $g_{\varepsilon} \in \mathcal{H}^{t}$ for any $t \in[s, s+\min \{\gamma, \alpha\}] \backslash \mathbb{Z}$. In a similar way, we may analyse the mixed derivatives $Y f:=Y_{1} \ldots Y_{k} f$, where $Y_{\ell} \in\left\{X_{i, j}: 1 \leqslant i<j \leqslant d\right\}, 1 \leqslant \ell \leqslant k$. In what follows, we provide the details.

For simplicity, we only consider powers of the same vector field $X$ but note that the exact same method would apply to a more general vector field $Y$ as in the previous paragraph. The equation satisfied by $X^{k} f$ is of the form

$$
X^{k} f=\sum_{\substack{\vec{k}:=\left(k_{1}, \ldots, k_{m+1}\right) \in \mathbb{N}_{0}^{m+1} \\ k_{1}+\cdots+k_{m+1}=k}} c_{\vec{k}} \mathrm{M}\left(X^{k_{1}} f, \ldots, X^{k_{m+1}} f\right),
$$

for some constants $c_{\vec{k}}>0$. Note that $X^{k_{j}} f \in \mathcal{H}^{1+\gamma}$ if $k_{j}<k$. Thus, we are led to splitting the sum in (6.10) into two parts, one of them containing precisely those summands that carry the term $X^{k} f$. There are $m+1$ of them, so

$$
X^{k} f=\sum_{\vec{k} \in K} c_{\vec{k}} \mathrm{M}\left(X^{k_{1}} f, \ldots, X^{k_{m+1}} f\right)+(m+1) \mathrm{M}\left(f, \ldots, f, X^{k} f\right),
$$

where $\left(k_{1}, \ldots, k_{m+1}\right) \in K$ if and only if $k_{j}<k$, for every $1 \leqslant j \leqslant m+1$, and $k_{1}+\cdots+k_{m+1}=k$. The first term on the right-hand side of (6.11) can be easily bounded in $\mathcal{H}^{1+\gamma}$ with (3.8), yielding

$$
\sum_{\vec{k} \in K} c_{\vec{k}}\left\|\mathrm{M}\left(X^{k_{1}} f, \ldots, X^{k_{m+1}} f\right)\right\|_{\mathcal{H}^{1+\gamma}} \lesssim\|f\|_{\mathcal{H}^{s}}^{m+1}
$$

\footnotetext{
${ }^{6}$ If $s+\delta=1$, then $\mathcal{H}^{S+\delta}$ is not defined; however, by using any $\delta^{\prime}<\delta$ in the reasoning above, the conclusion is that $g_{\varepsilon} \in \mathcal{H}^{t}$ for every $t<1$ and therefore $f \in \mathcal{H}^{t}$ for every $t<1$.
} 
To handle the second term, let $\varepsilon \in(0,1)$ and decompose $f=\varphi_{0}+\varphi_{1}, X^{k} f=\psi_{0}+\psi_{1}$, with $\varphi_{1}, \psi_{1} \in$ $C^{\infty}\left(\mathbb{S}^{d-1}\right)$ and $\left\|\varphi_{0}\right\|_{L^{2}}<\varepsilon\|f\|_{L^{2}},\left\|\psi_{0}\right\|_{L^{2}}<\varepsilon\left\|X^{k} f\right\|_{L^{2}}$. Because $f \in \mathcal{H}^{s}$, we have that $\varphi_{0} \in \mathcal{H}^{s}$ and $\psi_{0} \in \mathcal{H}^{\gamma}$. Now take $\delta \in(0,1)$ satisfying $\delta \leqslant \min \{\gamma, \alpha\}$; recall that $\gamma=s-\lfloor s\rfloor$ and that $\alpha$ was chosen immediately following (6.9). The equation satisfied by $\psi_{0}$ may be derived from (6.11). Applying $(\Theta-I)^{2}$ to both sides of that equation and invoking Lemma 6.2, we find that, if $\varepsilon>0$ is small enough, then

$$
\begin{aligned}
\|(\Theta-I)^{2} \psi_{0} & \left\|_{L^{2}\left(\mathbb{S}^{d-1}\right)} \lesssim \sum_{\vec{k} \in K} c_{\vec{k}}\right\|(\Theta-I)^{2} \mathrm{M}\left(X^{k_{1}} f, \ldots, X^{k_{m+1}} f\right) \|_{L^{2}\left(\mathbb{S}^{d-1}\right)} \\
& +\left(\left\|(\Theta-I)^{2} \varphi_{0}\right\|_{L^{2}\left(\mathbb{S}^{d-1}\right)}+\left\|(\Theta-I)^{2} \varphi_{1}\right\|_{L^{2}\left(\mathbb{S}^{d-1}\right)}\right)\left\|X^{k} f\right\|_{L^{2}\left(\mathbb{S}^{d-1}\right)} \\
& +\left(\left\|(\Theta-I) \varphi_{0}\right\|_{L^{2}\left(\mathbb{S}^{d-1}\right)}+\left\|(\Theta-I) \varphi_{1}\right\|_{L^{2}\left(\mathbb{S}^{d-1}\right)}\right)^{2}\left\|X^{k} f\right\|_{L^{2}\left(\mathbb{S}^{d-1}\right)} \\
& +\left(\left\|(\Theta-I) \varphi_{0}\right\|_{L^{2}\left(\mathbb{S}^{d-1}\right)}+\left\|(\Theta-I) \varphi_{1}\right\|_{L^{2}\left(\mathbb{S}^{d-1}\right)}\right)\left\|(\Theta-I) \psi_{0}\right\|_{L^{2}\left(\mathbb{S}^{d-1}\right)} \\
& +\left(\left\|(\Theta-I) \varphi_{0}\right\|_{L^{2}\left(\mathbb{S}^{d-1}\right)}+\left\|(\Theta-I) \varphi_{1}\right\|_{L^{2}\left(\mathbb{S}^{d-1}\right)}\right)\left\|(\Theta-I) \psi_{1}\right\|_{L^{2}\left(\mathbb{S}^{d-1}\right)} \\
& +C_{\varepsilon}|t|^{\delta}\left\|(\Theta-I) \psi_{0}\right\|_{L^{2}\left(\mathbb{S}^{d-1}\right)}+\left\|(\Theta-I)^{2} \psi_{1}\right\|_{L^{2}\left(\mathbb{S}^{d-1}\right)} .
\end{aligned}
$$

Consequently, by means of second differences, we obtain

$$
\sup _{0<|t| \leqslant 1}|t|^{-(\delta+\gamma)}\left\|(\Theta-I)^{2} \psi_{0}\right\|_{L^{2}\left(\mathbb{S}^{d-1}\right)}<\infty
$$

and, as a result, $\psi_{0} \in \mathcal{H}^{\gamma+\delta}$. It follows that $X^{k} f \in \mathcal{H}^{\gamma+\delta}$ and, because $X \in\left\{X_{i, j}: 1 \leqslant i<j \leqslant d\right\}$ was arbitrary, ${ }^{7} f \in \mathcal{H}^{s+\delta}$. The proof of the proposition is now complete.

\subsection{Second differences}

Given $s \in(0,2)$, we define the space $\mathscr{H}^{s}=\mathscr{H}^{s}\left(\mathbb{S}^{d-1}\right)$ of all functions $f \in L^{2}\left(\mathbb{S}^{d-1}\right)$, for which the norm

$$
\|f\|_{\mathscr{H} s}=\|f\|_{L^{2}(\mathbb{S} d-1)}+\sum_{1 \leqslant i<j \leqslant d} \sup _{|t| \leqslant 1}|t|^{-s}\left\|\left(e^{t X_{i, j}}-I\right)^{2} f\right\|_{L^{2}(\mathbb{S} d-1)}
$$

is finite. We see that

$$
\left(e^{t X_{i, j}}-I\right)^{2} f=f \circ e^{2 t X_{i, j}}-2 f \circ e^{t X_{i, j}}+f
$$

resembles a second difference of $f$. From the definition, it is immediate that $\|f\|_{\mathscr{H} s} \leqslant 2\|f\|_{\mathcal{H}^{s}}$ provided that $s \in(0,1)$, so $\mathcal{H}^{s} \subseteq \mathscr{H}^{s}$. The reverse inclusion also holds. Moreover, if $s \in(0,2) \backslash\{1\}$, then $\mathcal{H}^{s}=\mathscr{H}^{s}$, and the two norms given by (2.7) and (6.12) are equivalent. These assertions have all appeared in the literature; in what follows, we provide precise references.

Let us discuss the Euclidean case first. Given $s \in(0,1)$, we defined the Hölder space $\Lambda_{s}\left(\mathbb{R}^{d}\right)$ to contain precisely those functions $f: \mathbb{R}^{d} \rightarrow \mathbb{C}$ for which the norm

$$
\|f\|_{L^{\infty}\left(\mathbb{R}^{d}\right)}+\sup _{|t|>0}|t|^{-s}\|f(x+t)-f(x)\|_{L_{x}^{\infty}\left(\mathbb{R}^{d}\right)}
$$

is finite, whereas for $s=k+\delta, 1 \leqslant k \in \mathbb{N}, \delta \in(0,1)$, we have that $f \in \Lambda_{s}\left(\mathbb{R}^{d}\right)$ if $f \in C^{k}\left(\mathbb{R}^{d}\right)$ and $\partial^{\alpha} f \in \Lambda_{\delta}\left(\mathbb{R}^{d}\right)$, for all multi-indices $\alpha \in \mathbb{N}_{0}^{d}$ with $|\alpha|=k$. Given $s \in(0,2)$, consider the norm (defined in terms of second differences)

$$
\|f\|_{L^{\infty}\left(\mathbb{R}^{d}\right)}+\sup _{|t|>0}|t|^{-s}\|f(x+2 t)-2 f(x+t)+f(x)\|_{L_{x}^{\infty}\left(\mathbb{R}^{d}\right)}
$$

${ }^{7}$ Again, if $s+\delta \in \mathbb{Z}$, then the conclusion is that $f \in \mathcal{H}^{t}$ for every $t \in[0, s+\delta] \backslash \mathbb{Z}$. 
and the corresponding space of functions for which the latter norm is finite. These two spaces coincide if $s \in(0,2) \backslash\{1\}$, as dictated by the classical equivalence between Hölder and Zygmund spaces, the latter being defined through higher differences; precise references include [27, Ch. V, Prop. 8] and [30, Ch. 2, §2.6]. More generally, one may consider an $L^{p}$-norm in $x, 1 \leqslant p \leqslant \infty$, and possibly an additional $L^{q}$-norm in $t, 1 \leqslant q \leqslant \infty$; see [27, Ch. V, Prop. 8'] and [30, Ch. 2, §2.6].

For the case of the unit sphere $\mathbb{S}^{d-1}$, the equivalence between the $\mathcal{H}^{s}$ - and the $\mathscr{H}^{s}$-norms, and therefore the equality of the two corresponding spaces, can be found in $[15,16]$. These works rely on harmonic extensions, in a similar spirit to the aforementioned chapter in [27]. Of particular relevance are Propositions 4.1 and 4.3 in [15] and Proposition 1.8 in [16]. In the former article [15], the function space $\Lambda(\alpha ; p, q)$ is defined for $\alpha>0,1 \leqslant p, q \leqslant \infty$, and shown to be equivalent to a variant thereof using first- and secondorder differences; the special case $(p, q)=(2, \infty)$ and $\alpha=s \in(0,1)$ of this equivalence is used to establish that the spaces $\mathcal{H}^{s}$ and $\mathscr{H}^{s}$ coincide whenever $s \in(0,1)$. In the latter article [16], spaces of index $\alpha=k+\gamma, k \in \mathbb{N}$, are related to those of index $\gamma$ in a precise way; in turn, this is used to establish the equivalence between the spaces $\mathcal{H}^{s}$ and $\mathscr{H}^{s}$ whenever $s \in(1,2)$. It should be pointed out that the norms in terms of first and second differences considered in [15] are slightly different from the ones that we are using to define $\mathcal{H}^{s}$ and $\mathscr{H}^{s}$. However, the norms are seen to be equivalent; see [7, Cor. 3.11]. See also [5] and [23, Theorems 3.1 and 3.3].

We proceed to describe an alternative approach to the equivalence discussed in the previous paragraph that perhaps requires less effort from the unfamiliar reader.

Firstly, the equivalence between $\mathcal{H}^{s}$ and $\mathscr{H}^{s}$ when $s \in(0,1)$ follows directly from the combinatorial proof of [19, Lemma 1.1], stated in [19] for the case of $\mathbb{R}^{d}$. For the convenience of the reader, we provide a brief sketch of the argument. As mentioned already, the estimate $\|f\|_{\mathscr{H}^{s}} \leqslant 2\|f\|_{\mathcal{H}^{s}}$ follows easily from the definitions. For the reverse inequality, consider the following identity, which is valid for every $t \in \mathbb{R}, X \in\left\{X_{i, j}: 1 \leqslant i<j \leqslant d\right\}$ and $m \in \mathbb{N}$ :

$$
2^{m}\left(e^{t X}-I\right)=\left(e^{2^{m} t X}-I\right)-\sum_{i=0}^{m-1} 2^{m-1-i}\left(e^{2^{i} t X}-I\right)^{2}
$$

Applying this operator to a function $f \in \mathscr{H}^{s}$, taking the $L^{2}\left(\mathbb{S}^{d-1}\right)$-norm on both sides and invoking the triangle inequality yields

$$
2^{m}\left\|\left(e^{t X}-I\right) f\right\|_{L^{2}(\mathbb{S} d-1)} \leqslant\left\|\left(e^{2^{m} t X}-I\right) f\right\|_{L^{2}(\mathbb{S} d-1)}+\sum_{i=0}^{m-1} 2^{m-1-i}\left\|\left(e^{2^{i} t X}-I\right)^{2} f\right\|_{L^{2}(\mathbb{S} d-1)} .
$$

Dividing by $2^{m}$, using that $\left\|\left(e^{2^{m} t X}-I\right) f\right\|_{L^{2}\left(\mathbb{S}^{d-1}\right)} \leqslant 2\|f\|_{L^{2}\left(\mathbb{S}^{d-1}\right)} \leqslant 2\|f\|_{\mathscr{H}^{2}}$ and letting $m \rightarrow \infty$, we then obtain

$$
\left\|\left(e^{t X}-I\right) f\right\|_{L^{2}(\mathbb{S} d-1)} \leqslant \frac{1}{2} \sum_{i=0}^{\infty} 2^{-i}\left\|\left(e^{2^{i} t X}-I\right)^{2} f\right\|_{L^{2}(\mathbb{S} d-1)}
$$

Multiplying by $|t|^{-s}$ and taking the supremum over $t \in \mathbb{R}$ shows that, when $s<1$, the following holds:

$$
\begin{aligned}
\sup _{t \in \mathbb{R}}|t|^{-s}\left\|\left(e^{t X}-I\right) f\right\|_{L^{2}\left(\mathbb{S}^{d-1}\right)} & \leqslant \frac{1}{2}\left(\sum_{i=0}^{\infty} 2^{-i(1-s)}\right) \sup _{t \in \mathbb{R}}|t|^{-s}\left\|\left(e^{t X}-I\right)^{2} f\right\|_{L^{2}\left(\mathbb{S}^{d-1}\right)} \\
& =\frac{1}{2\left(1-2^{-(1-s)}\right)} \sup _{t \in \mathbb{R}}|t|^{-s}\left\|\left(e^{t X}-I\right)^{2} f\right\|_{L^{2}\left(\mathbb{S}^{d-1}\right)} .
\end{aligned}
$$


On the other hand, by $2 \pi$-periodicity of $e^{t X}$ and $S O(d)$-invariance of $\sigma_{d-1}$,

$$
\sup _{t \in \mathbb{R}}|t|^{-s}\left\|\left(e^{t X}-I\right)^{k} f\right\|_{L^{2}\left(\mathbb{S}^{d-1}\right)} \simeq \sup _{|t|<1}|t|^{-s}\left\|\left(e^{t X}-I\right)^{k} f\right\|_{L^{2}\left(\mathbb{S}^{d-1}\right)}
$$

for $k \in\{1,2\}$. This together with (6.13) yields $\|f\|_{\mathcal{H}^{s}} \leqslant C\|f\|_{\mathscr{H} s}$ for some $C<\infty$.

Secondly, the equivalence between $\mathcal{H}^{s}$ and $\mathscr{H}^{s}$ when $s \in(1,2)$ can be obtained via the techniques in [8, §3] (especially Theorem 3.6) and [7, §2.3], which rely on the modulus of smoothness and Marchaudtype inequalities. Indeed, the equivalence of the norms $\|\cdot\|_{\mathcal{W}_{p}^{r, \alpha}}$ and $\|\cdot\|_{H_{p}^{r+\alpha}}$ given by [8, Theorem 3.6] provides the answer after specialising to $(\ell, r p, \alpha)=(1,1,2, s-1)$, because in this case $\|f\|_{\mathcal{H}^{s}} \simeq$ $\|f\|_{W_{p}^{r, \alpha}}+\|f\|_{\mathcal{H}^{s-1}},\|f\|_{H_{p}^{r+\alpha}} \simeq\|f\|_{\mathscr{H}^{s}}$ and, as already remarked, $\|f\|_{\mathcal{H}^{s-1}} \simeq\|f\|_{\mathscr{H}^{s-1}} \lesssim\|f\|_{\mathscr{H}^{s}}$. The argument is straightforward but lengthy; thus, the reader is directed to the aforementioned references.

\subsection{One final remark}

Our proof of Theorem 1.1 does not in general handle the case when $\lambda=0$ in (1.12). An exception corresponds to the case when $m=2 k$ is an even integer, $\vec{k} \in\{0,1\}^{m+1}$ satisfies $k_{1}+\cdots+k_{m+1}=k-1$ and $a>0$ on $\mathbb{S}^{d-1}$ (or, more generally, $a=0$ on a set of $\sigma_{d-1}$-measure zero), which corresponds to the Euler-Lagrange equation (1.10) with $\lambda=0$. In this case, by multiplying both sides of (1.12) by $\bar{f}$ and integrating over $\mathbb{S}^{d-1}$, one concludes that $\left\|\widehat{f \sigma}_{d-1}\right\|_{L^{m+2}\left(\mathbb{R}^{d}\right)}=0$, which clearly forces $f=\mathbf{0}$. It remains unclear whether one should expect general solutions of (1.12) to be smooth when $\lambda=0$.

Acknowledgments. D.O.S. is supported by the EPSRC New Investigator Award 'Sharp Fourier Restriction Theory', Grant No. EP/T001364/1, and is grateful to Michael Christ, Giuseppe Negro and Po-Lam Yung for valuable discussions during the preparation of this work. The authors thank the anonymous referees for a careful reading of the article and valuable suggestions.

Conflict of Interest: None.

\section{References}

[1] E. Carneiro and D. Oliveira e Silva, 'Some sharp restriction inequalities on the sphere', Int. Math. Res. Not. IMRN 2015(17) (2015), 8233-8267.

[2] M. Christ and R. Quilodrán, 'Gaussians rarely extremize adjoint Fourier restriction inequalities for paraboloids', Proc. Amer. Math. Soc. 142(3) (2014), 887-896.

[3] M. Christ and S. Shao, 'Existence of extremals for a Fourier restriction inequality', Anal. PDE 5(2) (2012), 261-312.

[4] M. Christ and S. Shao, 'On the extremizers of an adjoint Fourier restriction inequality', Adv. Math. 230(3) (2012), 957-977.

[5] L. Colzani, 'Lipschitz spaces on compact rank one symmetric spaces', in Harmonic Analysis (Cortona, 1982), Lecture Notes in Mathematics, Vol. 992 (Springer, Berlin, 1983), 139-160.

[6] F. Dai and Y. Xu, 'Moduli of smoothness and approximation on the unit sphere and the unit ball', Adv. Math. 224(4) (2010), 1233-1310.

[7] F. Dai and Y. Xu, 'Polynomial approximation in Sobolev spaces on the unit sphere and the unit ball', J. Approx. Theory 163(10) (2011), 1400-1418.

[8] F. Dai and Y. Xu, Approximation Theory and Harmonic Analysis on Spheres and Balls, Springer Monographs in Mathematics (Springer, New York, 2013).

[9] L. Fanelli, L. Vega and N. Visciglia, 'On the existence of maximizers for a family of restriction theorems', Bull. Lond. Math. Soc. 43(4) (2011), 811-817.

[10] D. Foschi, On the Regularity of Multilinear Forms Associated to the Wave Equation (PhD Dissertation, Princeton University, 2000).

[11] D. Foschi, 'Global maximizers for the sphere adjoint Fourier restriction inequality', J. Funct. Anal. 268 (2015), 690-702.

[12] D. Foschi and D. Oliveira e Silva, 'Some recent progress on sharp Fourier restriction theory', Anal. Math. 43(2) (2017), 241-265.

[13] R. Frank, E. H. Lieb and J. Sabin, 'Maximizers for the Stein-Tomas inequality', Geom. Funct. Anal. 26 (2016), no. 4, 1095-1134.

[14] L. Grafakos, Modern Fourier Analysis, third ed., Graduate Texts in Mathematics, Vol. 250 (Springer, New York, 2014).

[15] H. C. Greenwald, 'Lipschitz spaces on the surface of the unit sphere in Euclidean n-space', Pacific J. Math. 50 (1974), 63-80.

[16] H. C. Greenwald, 'Lipschitz spaces of distributions on the surface of unit sphere in Euclidean n-space', Pacific J. Math. 70(1) (1977), 163-176. 
[17] B. Hall, Lie Groups, Lie Algebras, and Representations. An Elementary Introduction, second ed., Graduate Texts in Mathematics, Vol. 222 (Springer-Verlag, New York, 2015).

[18] E. Hebey, Nonlinear Analysis on Manifolds: Sobolev Spaces and Inequalities, Courant Lecture Notes in Mathematics, Vol. 5 (New York University, Courant Institute of Mathematical Sciences, New York; American Mathematical Society, Providence, RI, 1999).

[19] C. S. Herz, 'Lipschitz spaces and Bernstein's theorem on absolutely convergent Fourier transforms', J. Math. Mech. 18 (1968-69), 283-323.

[20] L. Hörmander, 'Hypoelliptic second order differential equations', Acta Math. 119 (1967), 147-171.

[21] V. Jurdjevic and H. J. Sussmann, 'Control systems on Lie groups', J. Differ. Equ. 12 (1972), 313-329.

[22] J.-L. Lions and E. Magenes, Non-homogeneous Boundary Value Problems and Applications: Vol. I. Die Grundlehren der mathematischen Wissenschaften [Comprehensive Studies in Mathematics], Band 181 (Springer-Verlag, New York, 1972). Translated from the French by P. Kenneth.

[23] P.I. Lizorkin and KH. P. Rustamov, 'Nikol'ski1̌-Besov spaces on the sphere in connection with approximation theory', Proc. Steklov Inst. Math. 204 (1994), 149-172.

[24] D. Oliveira e Silva and R. Quilodrán, 'Global maximizers for adjoint Fourier restriction inequalities on low dimensional spheres', J. Funct. Anal. 280(7) (2021) 108825, https://doi.org/10.1016/j.jfa.2020.108825.

[25] S. Shao, 'On existence of extremizers for the Tomas-Stein inequality for $\mathbb{S}^{1}$, , J. Funct. Anal. 270 (2016), $3996-4038$.

[26] S. Shao, 'On smoothness of extremizers of the Tomas-Stein inequality for $\mathbb{S}^{1}$ ', Preprint (2016), arXiv:1601.07119v3.

[27] E. M. Stein, Singular Integrals and Differentiability of Functions (Princeton University Press, Princeton, NJ, 1970).

[28] E. M. Stein, Harmonic Analysis: Real-Variable Methods, Orthogonality, and Oscillatory Integrals (Princeton University Press, Princeton, NJ, 1993).

[29] P. Tomas, 'A restriction theorem for the Fourier transform', Bull. Amer. Math. Soc. 81(2) (1975), 477-478.

[30] H. Triebel, Theory of Function Spaces II, Monographs in Mathematics, Vol. 84 ( Birkhäuser Verlag, Basel, Switzerland, 1992). 